\title{
NUMERICAL INVESTIGATION OF THE EFFECT OF CHIRALITY OF CARBON NANOTUBE ON THE INTERFACIAL THERMAL RESISTANCE
}

\author{
A Thesis \\ by \\ YUZHU HU \\ Submitted to the Office of Graduate and Professional Studies of \\ Texas A\&M University \\ in partial fulfillment of the requirements for the degree of \\ MASTER OF SCIENCE
}

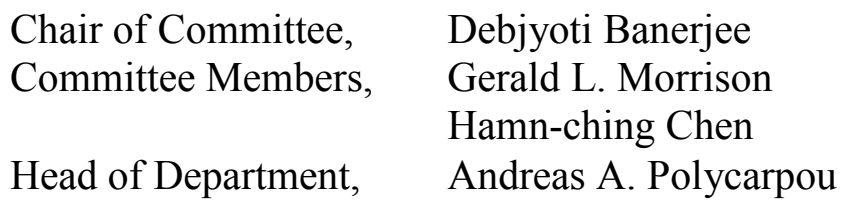

August 2014

Major Subject: Mechanical Engineering

Copyright 2014 Yuzhu Hu 


\begin{abstract}
Concentrated Solar Power (CSP) systems are used widely as a stable and reliable renewable source of energy. However, intermittency of this power source and the variability in demand for electrical power creates challenges that necessitate the integration with energy storage for reliable dispatch of power. Thermal Energy Storage (TES) systems provide a cheap, cost-effective and reliable option for energy storage in renewable power delivery systems. Due to their low vapor pressures at elevated temperatures, molten salts and their eutectics are used in conventional high temperature thermal energy storage (TES) systems and also as coolants for energy conversion, such as in power tower configurations that are typically used in CSP applications. A major drawback of the molten salts is their relatively poor thermo-physical properties, which may lead to lower systemic efficiencies in CSP/TES. Recent reports in the literature have shown that doping molten salts with nanoparticles at minute concentrations (typically less than 5\% mass fraction and ideally at less than 1-2\% mass fraction) can significantly enhance the thermo-physical properties of these nanomaterial (also termed as "nanocomposites" in solid state and "nanofluids" in liquid state). The dominant factor that controls the resultant thermo-physical properties of these nanomaterials is the interfacial thermal resistance (or Kapitza Resistance " $R_{k}$ ") that impedes the heat transfer between the nanoparticle surface and the bulk solvent molecules.
\end{abstract}


In this study, the interfacial thermal resistance between a carbon nanotube (CNT) and carbonate molten salt eutectics were calculated by using numerical models that were then implemented in Molecular Dynamics (MD) simulations. The estimates for " $R_{k}$ " obtained from these simulations enabled the prediction of the optimum dimensions of the nanoparticles for maximizing the thermo-physical properties of the mixture, i.e. thermal conductivity and specific heat capacity values of these nanomaterial. The simulations were restricted to the carbonate salt eutectic, which is composed of a molar ratio of $62: 38$ for lithium carbonate $\left(\mathrm{Li}_{2} \mathrm{CO}_{3}\right)$ and potassium carbonate $\left(\mathrm{K}_{2} \mathrm{CO}_{3}\right)$. In this study, parametric simulations were performed to estimate the values of " $R_{k}$ " by varying the chirality of a single walled CNT (i.e, for armchair, chiral, and zig-zag CNT). The results show that the Kapitza resistance of the CNT is significantly affected by the change in the chirality of the CNT. 


\section{DEDICATION}

To my dear husband, parents — for their support of all that I do 


\section{ACKNOWLEDGEMENTS}

For those people who have supported the creation of this work, it is hard for me to choose the words that can truly and accurately express my heartfelt gratitude and appreciation.

I would like to express my gratitude to Dr. Debjyoti Banerjee, Professor of the Department of Mechanical Engineering, for his advising, encouragement and comments during the coursework. Through his guidance and help, not only an advisor for guiding the research but also acted as a family member for caring for me. I would like to thank Dr. Gerald L. Morrison and Dr. Hamn-ching Chen for serving on the thesis advisory committee.

I would like to acknowledge the assistance from Dr. Lisa M. Pérez at the Laboratory for Molecular Simulations (LMS) at Texas A\&M University, the Texas A\&M University Supercomputing Center (SC), and the Texas A\&M Engineering Experiment Station (TEES) for use of their resources and making this study possible. I would also like to acknowledge financial support from the following sources during the execution of this study: DARPA Micro/Nano-Fluidics Fundamental Focus Center (DARPA-MF³), 3M Non-Tenured Faculty Fellowship, DOE Solar Energy Technologies Program 
(DOE/SETP), the Office of Naval Research (ONR), Qatar National Research Foundation (QNRF) and Dr. Sumanta Acharya at the National Science Foundation (under NSF Grant No. CBET-1134424).

I would also like to acknowledge Dr. Hongjoo Yang, Dr. Jiwon Yu, Dr. Byeongnam Jo and other members in Multi Phase Flow \& Heat Transfer Laboratory for their invaluable help for this work.

I would like to express my deepest appreciation to my husband, parents, friends and everyone who have inspired and supported me more than they will ever know. 


\section{NOMENCLATURE}

\begin{tabular}{|c|c|}
\hline A & surface area of nanoparticle \\
\hline $\mathrm{a}$ & radius of primary nanoparticle \\
\hline$a_{a}$ & radius of nanoparticle aggregation \\
\hline $\mathrm{C}$ & heat capacity $[\mathrm{J} / \mathrm{K}]$ \\
\hline $\mathrm{c}_{\mathrm{p}}$ & specific heat capacity $[\mathrm{J} / \mathrm{g} \cdot \mathrm{K}]$ \\
\hline $\mathrm{E}$ & intermolecular interaction energy $[\mathrm{kcal} / \mathrm{mole}]$ \\
\hline $\mathrm{K}_{\mathrm{s}}$ & bonding coefficient (stretching) $\left[\mathrm{kcal} / \mathrm{mole} \cdot \AA^{2}\right]$ \\
\hline $\mathrm{K}_{\mathrm{b}}$ & bonding coefficient (bending) $\left[\mathrm{kcal} /\right.$ mole $\left.\operatorname{radian}^{2}\right]$ \\
\hline $\mathrm{K}_{\mathrm{t}}$ & bonding coefficient (torsion) $[\mathrm{kcal} / \mathrm{mole}]$ \\
\hline $\mathrm{m}$ & $\operatorname{mass}[\mathrm{g}]$ \\
\hline $\mathrm{q}_{\mathrm{i}}, \mathrm{q}_{\mathrm{j}}$ & charge $[\mathrm{C}]$ \\
\hline$\Delta \mathrm{q}$ & heat flow difference $[\mathrm{mW}]$ \\
\hline$c_{\mathrm{p}}$ & specific heat capacity $[\mathrm{J} / \mathrm{g} \cdot \mathrm{K}]$ \\
\hline $\mathrm{R}$ & interfacial thermal resistance $\left[\mathrm{m}^{2} \cdot \mathrm{K} / \mathrm{W}\right]$ \\
\hline $\mathrm{r}$ & distance $[\AA]$ \\
\hline $\mathrm{r}_{0}$ & equilibrium bond distance $[\AA]$ \\
\hline $\mathrm{r}_{\mathrm{m}}$ & distance at which potential energy is minimum $[\AA]$ \\
\hline $\mathrm{T}$ & temperature $\left[\mathrm{K}\right.$ or $\left.{ }^{\circ} \mathrm{C}\right]$ \\
\hline
\end{tabular}


$\mathrm{t}$

$\mathrm{u}$

V

$\varepsilon$

$\phi$

$\eta$

$[\eta]$

$\varphi_{\mathrm{m}}$

$\varphi_{\mathrm{a}}$

$\theta$

$\theta_{0}$

$\rho$

$\sigma$

$\tau$
$\mathrm{T} / 1000[\mathrm{~K}]$

potential energy

volume of nanoparticle

depth of potential well [kcal/mole]

volume fraction of nanoparticle

viscosity $[\mathrm{Pa} \cdot \mathrm{s}]$

intrinsic viscosity [1/volume fraction]

maximum concentration

effective volume fraction of aggregations

bond angle [radian]

equilibrium value of the angle [radian]

density $\left[\mathrm{g} / \mathrm{cm}^{3}\right]$

distance at which potential energy is equal to zero $[\AA]$

time constant $[\mathrm{s}]$ 


\section{TABLE OF CONTENTS}

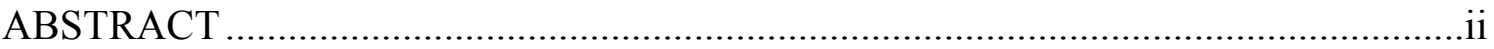

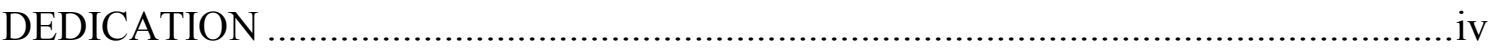

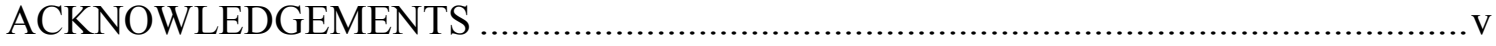

NOMENCLATURE ...................................................................................... vii

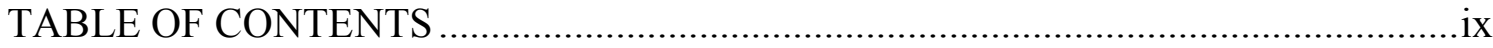

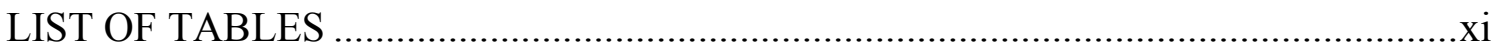

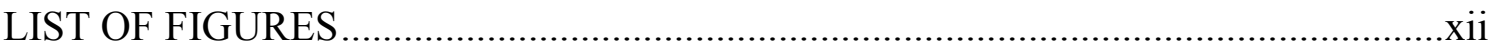

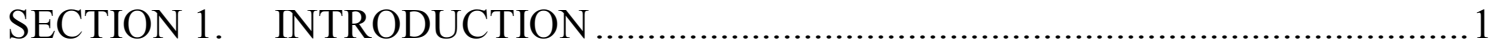

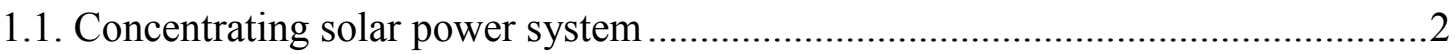

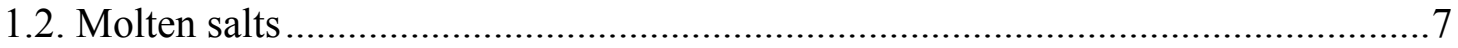

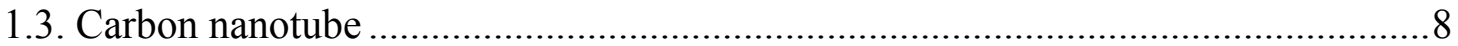

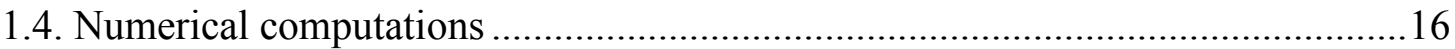

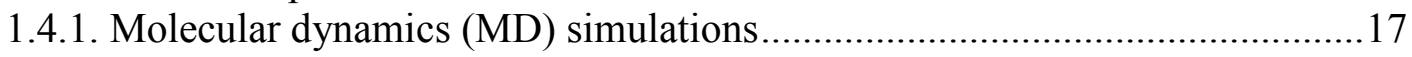

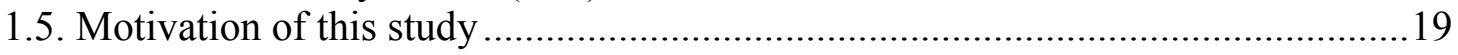

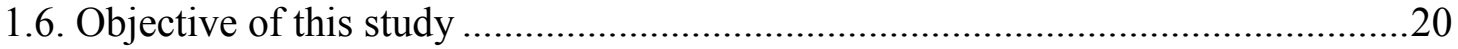

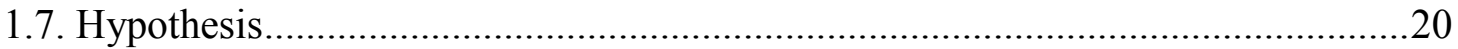

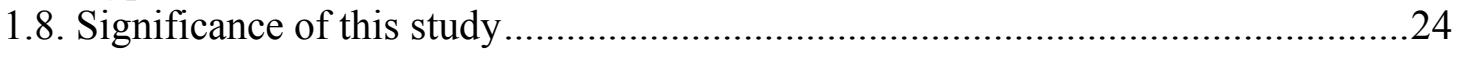

SECTION 2. LITERATURE REVIEW ...........................................................26

2.1. Prior investigations at the multi-phase flows and heat transfer laboratory ......27

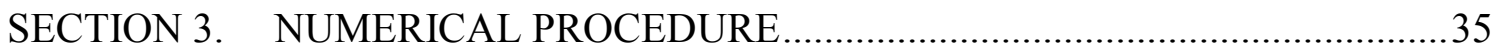

3.1. Carbon nanotube and molten salt used in numerical computations......................35 


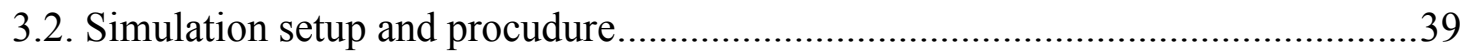

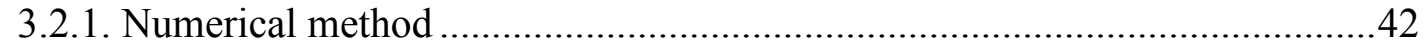

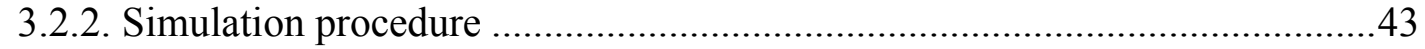

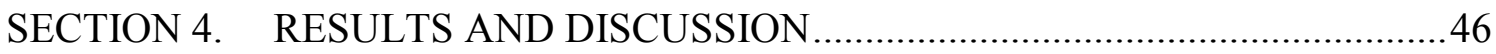

4.1. Interfacial thermal resistance and critical particle size of armchair SWCNT .......49

4.2. Interfacial thermal resistance and critical particle size of zig-zag SWCNT ..........53

4.3. Interfacial thermal resistance and critical particle size of chiral SWCNT .............58

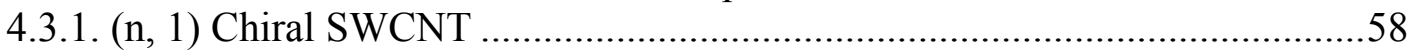

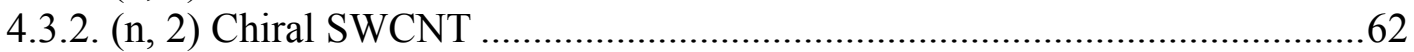

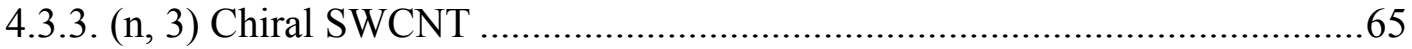

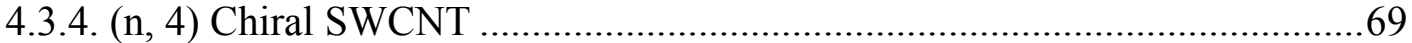

4.3.5. Defects induced in chiral SWCNT during MD Simulations...........................73

SECTION 5. SUMMARY AND CONCLUSION ………………..............................

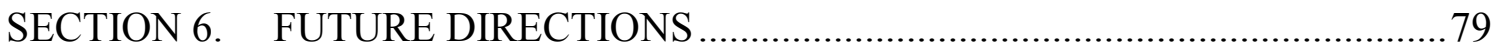

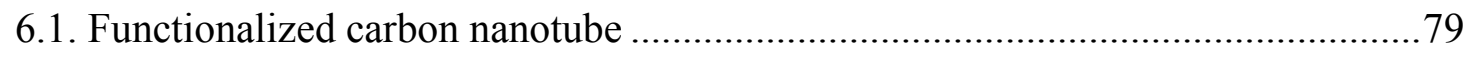

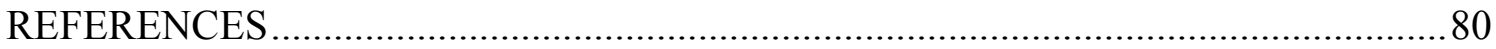




\section{LIST OF TABLES}

Page

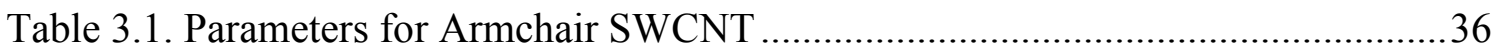

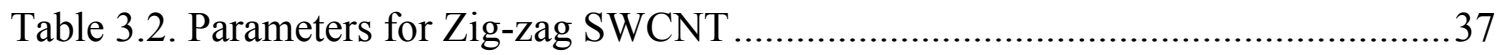

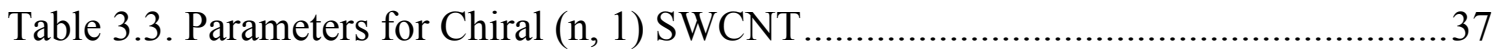

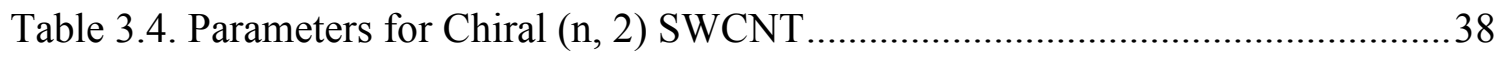

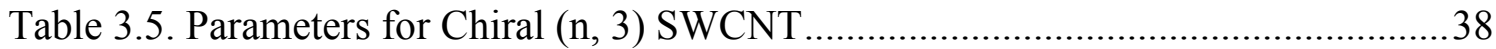

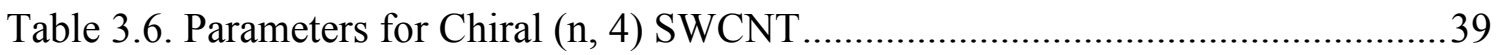

Table 3.7. Values of parameters used in the Molecular dynamics (MD) simulations .....39

Table 4.1. Interfacial thermal resistance and critical size of Armchair SWCNTs ...........50

Table 4.2. Interfacial thermal resistance and critical size of Zig-zag SWCNTs .............54

Table 4.3. Interfacial thermal resistance and critical particle of Chiral $(\mathrm{n}, 1) \mathrm{SWCNT} . .59$

Table 4.4. Interfacial thermal resistance and critical particle size of Chiral $(\mathrm{n}, 2)$

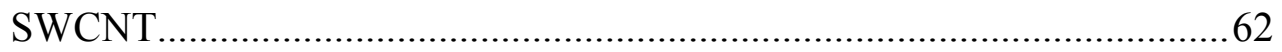

Table 4.5. Interfacial thermal resistance of Chiral $(\mathrm{n}, 3)$ SWCNT ................................66

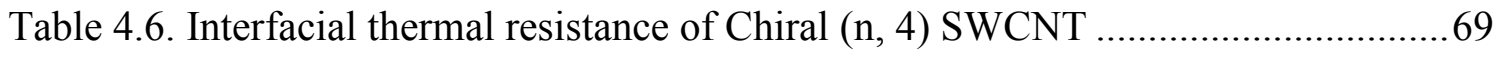




\section{LIST OF FIGURES}

Figure 1.1 Schematic showing the integration of storage in CSP plants. ${ }^{3}$

Figure 1.2 Plot comparing the diurnal variation of insolation (supply side) and demand for electrical power on a typical day based on monthly average.

Figure 1.3 Schematic of Concentrating Solar Power (CSP) unit. ${ }^{4}$

Figure 1.4. TEM images ${ }^{8-10}$ of different CNT -- A: SWCNT; B: MWCNT with different layers of graphene sheets: 5,2 and $7 .^{11}$

Figure 1.5. Schematic diagram showing how a hexagonal sheet of graphene is rolled to form a CNT with different chirality (A: armchair; B: zigzag; C: chiral). ${ }^{9,11-13}$

Figure 1.6 Images depicting various embodiments of an armchair SWCNT. ...............10

Figure 1.7 Images depicting various embodiments of a zig-zag SWCNT....................11

Figure 1.8 Images depicting various embodiments of two different chiral SWCNT.......12

Figure 1.9 Schematic depicting the vector configuration that is used to specify the chirality of a carbon nanotube for 2D graphene sheet. The pairs of integers $(\mathrm{n}, \mathrm{m})$ in the figure specify chiral vectors $\boldsymbol{C}_{\boldsymbol{h}}$ for carbon nanotubes, including zig-zag, armchair, and chiral tubules. Circled 
dots denote metallic tubules and the small dots are for semiconducting tubules. $^{12}$

Figure 1.10 Comparison between the structures of SWCNT with different chirality. Each image is shown with different length scales and magnification (please refer to the listed information specifying the morphology of each SWCNT).

Figure 1.11 High resolution tunneling electron microscope (HRTEM) image of sapphire/liquid aluminum mixture. ${ }^{28}$

Figure 1.12 Equilibrium structure snapshot for n-Tridecane (scale in $\AA$ )......................24

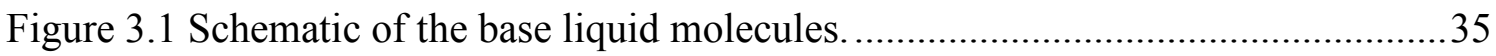

Figure 3.2. Schematic of carbon nanotube crystal structure with different chirality values used in this study. ${ }^{11}$

Figure 3.3. Simulation domain showing $(5,5)$ armchair SWCNT placed in the center and the solvent molecules of carbonate salt eutectic located around the crystal lattice of nanoparticle.

Figure 3.4. Transient temperature profile of a SWCNT obtained from the MD simulations.

Figure 4.1 Simulation domain showing $(5,5)$ armchair SWCNT placed in the center and the solvent molecules of carbonate salt eutectic located around the crystal lattice of the nanotube.

Figure 4.2 Spatial density distribution around a $(5,5)$ armchair SWCNT that is surrounded by molten salts molecules. 
Figure 4.3 Temperature decay plot of a $(5,5)$ armchair SWCNT that is surrounded by molten salts molecules.

Figure 4.4 Plot of interfacial thermal resistance of Armchair SWCNTs.

Figure 4.5 Simulation domain showing a $(10,0)$ zig-zag SWCNT placed in the center and the solvent molecules of carbonate salt eutectic located around the crystal lattice of the nanotube.

Figure 4.6 Spatial density distribution around a $(10,0)$ zig-zag SWCNT that is surrounded by molten salts molecules.

Figure 4.7 Temperature decay plot for a $(10,0)$ zig-zag SWCNT that is surrounded by molten salts molecules.

Figure 4.8 Plot of interfacial thermal resistance of Zig-zag SWCNTs.

Figure 4.9 Comparison of interfacial thermal resistance between armchair and Zig-zag (metallic) SWCNTs.

Figure 4.10 Simulation domain showing a $(10,1)$ chiral SWCNT placed in the center and the solvent molecules of carbonate salt eutectic located around the crystal lattice of the nanotube.

Figure 4.11 Spatial density distribution around a $(10,1)$ chiral SWCNT that is surrounded by molten salts molecules.

Figure 4.12 Temperature decay plot for a $(10,1)$ chiral SWCNT that is surrounded by molten salts molecules.

Figure 4.13 Plot of interfacial thermal resistance of $(n, 1)$ Chiral SWCNTs. 
Figure 4.14 Simulation domain showing a $(8,2)$ chiral SWCNT placed in the center and the solvent molecules of carbonate salt eutectic located around the crystal lattice of a nanotube.

Figure 4.15 Spatial density distribution around a $(8,2)$ chiral SWCNT that is surrounded by molten salts molecules.

Figure 4.16 Temperature decay plot for a $(8,2)$ chiral SWCNT that is surrounded by molten salts molecules.

Figure 4.17 Plot of interfacial thermal resistance of $(n, 2)$ Chiral SWCNTs.

Figure 4.18 Simulation domain showing $(7,3)$ chiral SWCNT placed in the center and the solvent molecules of carbonate salt eutectic located around the crystal of a nanotube.

Figure 4.19 Spatial density distribution around a $(7,3)$ chiral SWCNT that is surrounded by molten salts molecules.

Figure 4.20 Temperature decay plot of a $(7,3)$ chiral SWCNT that is surrounded by molten salts molecules.

Figure 4.21 Plot of interfacial thermal resistance of (n, 3) Chiral SWCNTs. 68

Figure 4.22 Simulation domain showing a $(7,4)$ chiral SWCNT placed in the center and the solvent molecules of carbonate salt eutectic located around the crystal lattice of a nanotube.

Figure 4.23 Spatial density distribution of a $(7,4)$ chiral SWCNT that is surrounded by molten salts molecules. 
Figure 4.24 Temperature decay plot of a $(7,4)$ chiral SWCNT that is surrounded by molten salts molecules. .72

Figure 4.25 Plot of interfacial thermal resistance of $(n, 4)$ Chiral SWCNTs..................72

Figure 4.26 Simulation domain showing a $(6,5)$ chiral SWCNT placed in the center and surrounded by solvent molecules of carbonate salt eutectic that are located around the crystal lattice of a nanotube.

Figure 4.27 Spatial density distribution for a $(6,5)$ chiral SWCNT that is surrounded by molten salts molecules.

Figure 4.28 Simulation domain showing a $(7,6)$ chiral SWCNT placed in the center and surrounded by solvent molecules of carbonate salt eutectic that are located around a crystal lattice of a nanotube.

Figure 4.29 Spatial density distribution for a $(7,6)$ chiral SWCNT that is surrounded by molten salts molecules. 


\section{SECTION 1. INTRODUCTION}

A senior research fellow emeritus in physics at Corpus Christi College, Oxford, named P. E. Hodgson, predicted in 2008 that every 14 years the world energy consumption will double. Also, the global demand for energy is increasing at a faster rate than supply side is growing to meet the demand. P.E. Hodgson claimed that the production of fossil fuels, such as oil or petroleum, in the whole world is expected to peak in ten years and fall thereafter. This may be offset by new discoveries, although no large oilfields have been discovered since $1980{ }^{1}$

Higher price of the hydrocarbon supplies caused by diminishing supply will lead to increase in demand for alternative energy sources, such as renewable energy. The cost of energy harvested from renewable sources (solar, wind, etc.) and artificial gasoline are currently not cost-competitive with energy harvested from fossil fuels. However, the rising price anticipated for fossil fuels coupled with advances in energy harvesting technologies are expected to provide favorable conditions for these non-conventional sources of energy to have a cost advantage and therefore wider adoption in society.

By considering the diminishing hydrocarbon resource and the higher expense of the current technologies for harvesting alternative energy sources, contemporary research 
activities pertaining to alternative energy has garnered significant attention. Nuclear, hydroelectric, solar, wind and geothermal energy are the most popular renewable and alternative resources that have garnered attention from contemporary researchers. Hence this work is mainly focused on solar thermal energy and other transformative applications associated with heat transfer involving nanoparticles as well as nanostructured surfaces (such as in cooling technologies and thermal management).

\subsection{Concentrating solar power system}

Solar energy, as one of the renewable energy resource, is considering as unlimited resource with minimal footprint on the environment. Contemporary solar energy harvesting technologies, utilize either photovoltaics (PV) or thermal power generation involving Concentrated Solar Power (CSP). PV is a direct method that utilizes semiconductor materials to directly convert the solar energy (solar spectrum or the electro-magnetic radiation in the sunlight) to electricity. In CSP technology solar receivers (mirrors or lenses) are used to focus or concentrate the solar thermal energy to achieve high temperature in the illuminated object (i.e., a solar tower). The high temperature energy source achieved in CSP is then utilized for electricity generation by utilizing thermodynamic cycles, such as Rankine cycle or Stirling cycle. The advantage of CSP systems accrues from the better reliability of operation in face of the intermittent nature of the energy source (i.e. diurnal cycles of the sun) and the cheap as well as cost-effective thermal energy storage (TES) platforms that are typically integrated with 
CSP generation units. In contrast for PV - direct storage of electrical energy is more costly (typically an order of magnitude higher than TES).

Solar energy generation enables numerous benefits such as free source of energy, abundant, and minimal impact on the environment. However, solar energy technologies need to overcome several techno-economic barriers. The intermittent source of supply from sun (due to day/night diurnal cycles and variation of insolation as the day progresses as well as due to adverse weather conditions such as due to cloud cover, rain, or snow) is an impediment to reliable operation and degradation in systemic efficiencies. In addition, the peak in demand for electrical power for typical civic entities has a phase lag with respect to the peak in insolation. This mismatch in the time of the peak in demand (which occurs between 4-7 pm) for electrical power for utilities compared to the peak in insolation in a typical day is shown in Figure 1.1 and Figure 1.2. Therefore, thermal energy storage system (TES), are typically used to levelize the difference in timing of the peak in supply with the peak in demand of electrical power. In most applications, the TES is typically used to absorb the excess power produced during peak in insolation (typically between 12 noon $-3 \mathrm{pm}$ ) and deliver the stored energy to match the peak in demand for electricity (typically between $4-7 \mathrm{pm}$ ). Considering the levelized cost of electricity (LCOE), CSP is actually cost-competitive with the contemporary peak price of electricity considering the diurnal price variation for electricity supplied by the utilities. ${ }^{2}$ Figure 1.3 shows the typical layout of a CSP unit. 


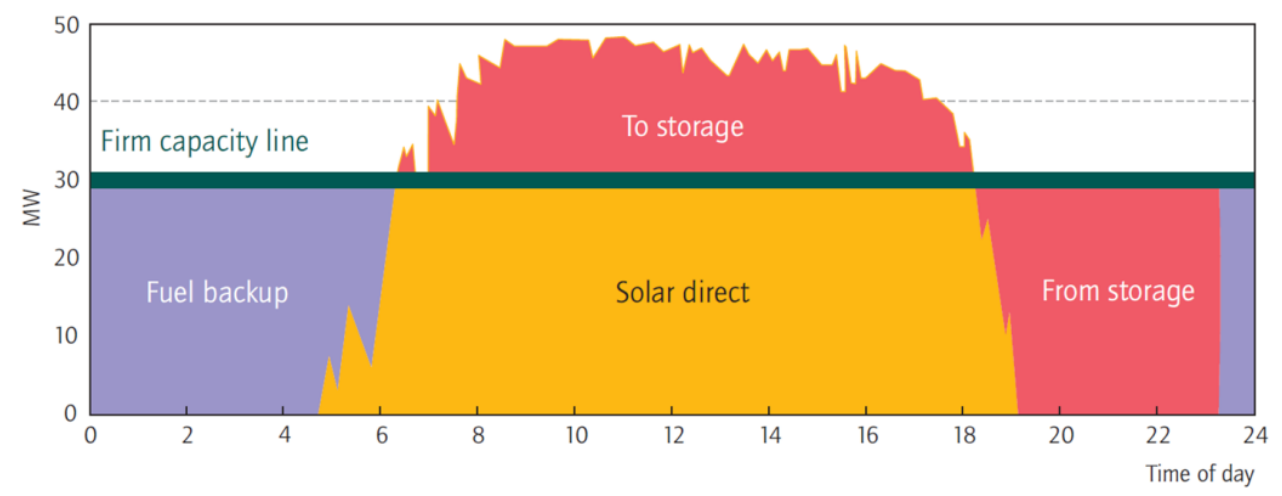

Figure 1.1 Schematic showing the integration of storage in CSP plants. ${ }^{3}$

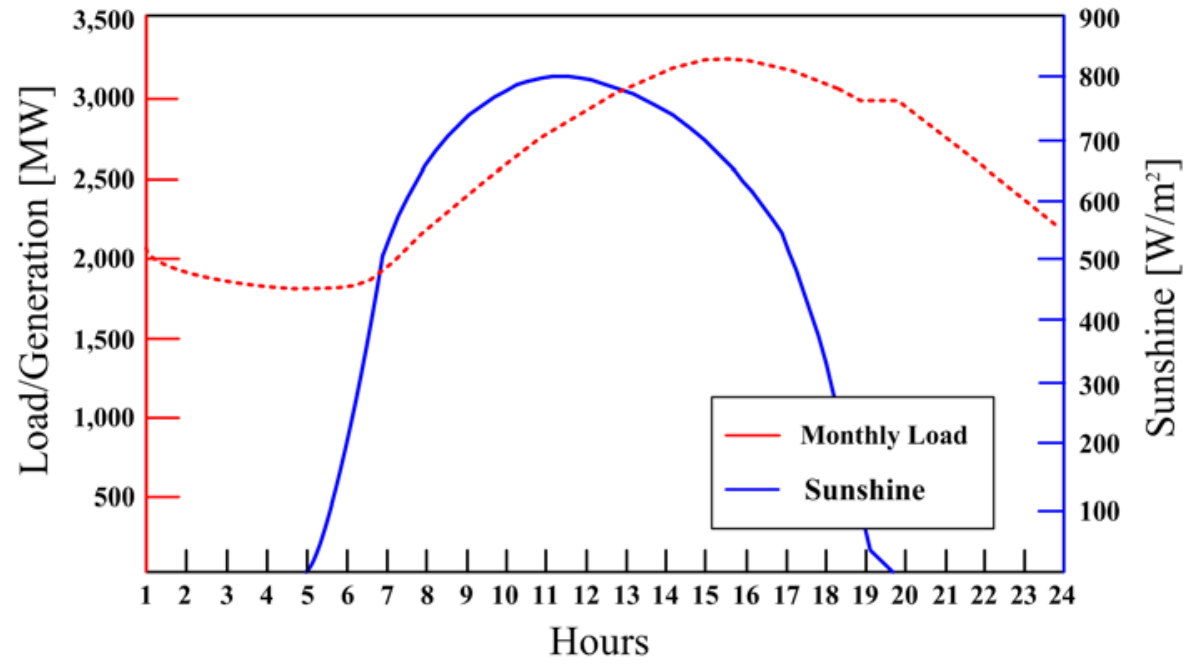

Figure 1.2 Plot comparing the diurnal variation of insolation (supply side) and demand for electrical power on a typical day based on monthly average. ${ }^{3}$ 


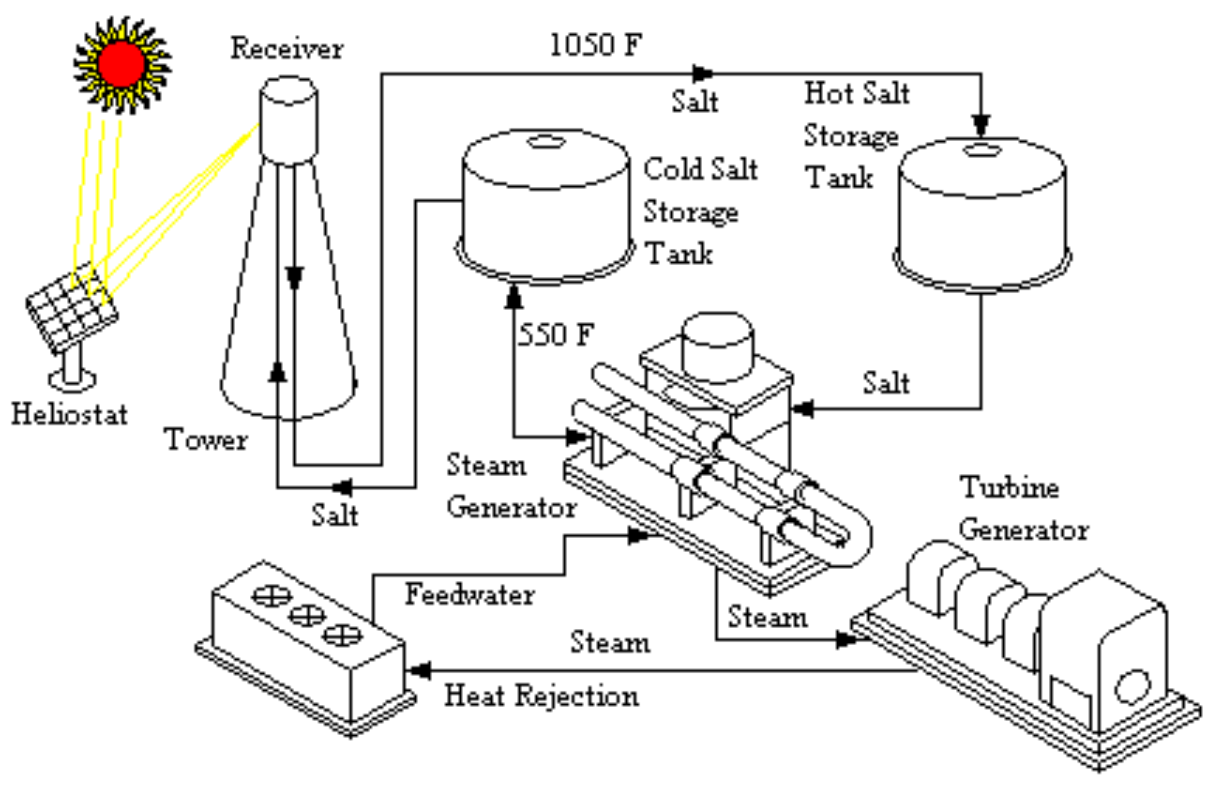

Figure 1.3 Schematic of Concentrating Solar Power (CSP) unit. ${ }^{4}$

Figure 1.3 shows the typical layout of a CSP unit involving a two-tank storage system and solar tower configuration. An array of solar receivers, such as lenses, mirrors, or heliostats, are used for collecting and focusing the incident solar thermal energy on to a solar tower (or other similar collection units such as pipes in a solar trough system). A heat transfer fluid (HTF) system is typically used to transfer the collected solar thermal energy from the solar tower. The thermal energy collected by the heat transfer fluid (HTF) can be used directly for power generation using a heat-engine operating in a thermodynamic cycle or the excess energy can be transferred to a storage unit (such as a hot storage tank or the warmer part of a thermocline storage unit which is usually the upper part of the thermocline storage unit). The hot storage tank (TES) typically contains molten salt maintained at a temperature which matches the temperature of the HTF. The 
storage tank is typically maintained at $\sim 1000{ }^{\circ} \mathrm{F}$. The thermal energy stored in the hot storage tank is then transferred using a heat exchanger to generate electrical power in a heat engine undergoing a thermodynamic cycle. For systems utilizing a steam power cycle (Rankine Cycle) the heat exchanger unit is used to generate steam from the hot molten salt. The stem is then used for driving a turbine to generate electricity (known as the power conversion module). The power conversion module is typically a turbine (i.e., operating on Rankine cycle) or an engine utilizing air-cycle (i.e., operating on Stirling cycle). The molten salt exiting the heat exchanges is at a lower temperature compared to the inlet to the heat exchanger. The salt at the lower temperature exiting the heat exchanger is stored in a "cold" storage tank (which is typically maintained at 500 $\left.{ }^{\circ} \mathrm{F}\right)$. The molten salt is then pumped back to the receiver (i.e., power tower) from the lower temperature storage tank.

The cost associated with the construction and operation of the thermal energy storage tank is typically $70 \%$ of the total cost of solar thermal power generation over the life-time of the CSP plant. Hence, any modifications or efficiency improvement that can be achieved for a solar thermal storage facility will definitely have a significant impact on the viability of the CSP units. Typically, the thermodynamic efficiency of the Rankine cycle mainly relies on the difference in temperature between the entry of the turbine (hot) and the condenser (cold). Since it is very expensive to decrease the condenser temperature, increasing the entry temperature is the key for enhancing the overall system efficiency of a typical CSP unit. The operating temperature is limited 
by the material properties of the TES. Hence innovation in material development may lead to development of CSP units with better energy efficiency can have a significant impact on solar thermal power generation technologies.

\subsection{Molten salts}

Molten salts are considered attractive materials for TES due to the wide range of the melting points (from $200{ }^{\circ} \mathrm{C}$ to $600{ }^{\circ} \mathrm{C}$ ) and their stability at high temperatures (molten salts are typically stable up to $\left.600{ }^{\circ} \mathrm{C}\right) .{ }^{5}$ There are various type of the molten salts, such as alkali-nitrate, alkali-carbonate, alkali-chloride (or halogen derivatives), or eutectic mixture of those. Molten salts confer several advantages as TES materials. ${ }^{6}$ First of all, the stability at high temperatures enables the operating temperature of the CSP to be increased to higher values (than compared to say oils) and consequently this enhances the efficiency of the thermodynamic cycle. Also compared to the cost of the conventional TES materials (such as oils), the molten salts are cheaper, considering the high volume of usage of TES materials, this helps reduce the fixed-cost of the CSP system by a significant margin. Last but not the least, the molten salts are deemed to be harmless for the environment and with proper handling they are considered to be non-toxic for human health - therefore their environmental footprint is expected to be minimal. This will help to obviate the cost for environmental remediation that is associated with any project life cycle analysis. However, the major drawback for molten salts is the poor thermo-physical properties (such as thermal conductivity, 
specific heat capacity, etc.) when compared to other conventional TES materials such as water or oils. ${ }^{5,7}$

\subsection{Carbon nanotube}

After discovery of carbon nanotube (CNT) a wide range of research topics have been sparked with focus on various industrial applications. ${ }^{8}$ Carbon nanotubes are classified as single-walled carbon nanotubes (SWCNT) and multi-walled carbon nanotubes (MWCNT). ${ }^{9}$ SWCNT consist of a single graphene layer rolled up into a seamless cylinder as shown in Figure 1.4A. On the other hand, as shown in Figure 1.4B, MWCNT consists of two or more concentric cylindrical shells of graphene sheets coaxially arranged around a central hollow core. Atomic forces such as van der Waals forces and ionic interactions between adjacent layers in MWCNT can provide unique and tunable materials properties..$^{8-10}$

A

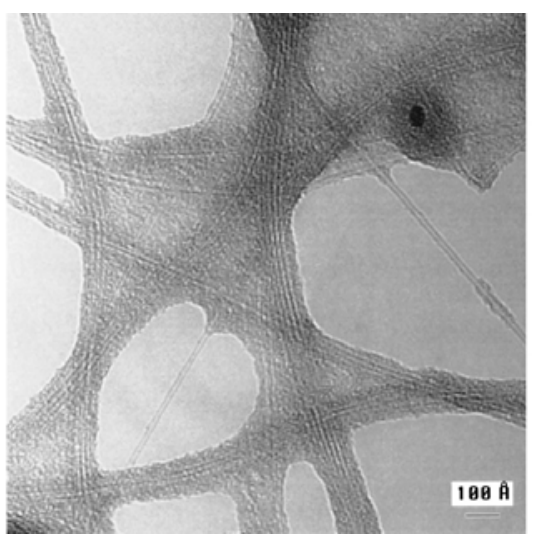

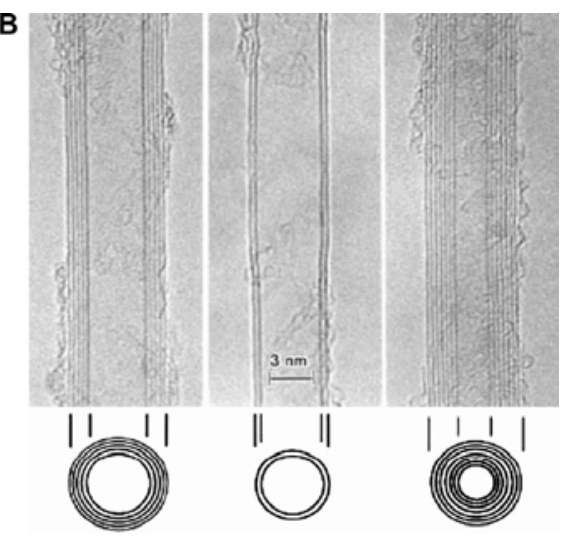

Figure 1.4. TEM images ${ }^{8-10}$ of different CNT -- A: SWCNT; B: MWCNT with different layers of graphene sheets: 5,2 and $7 .^{11}$ 

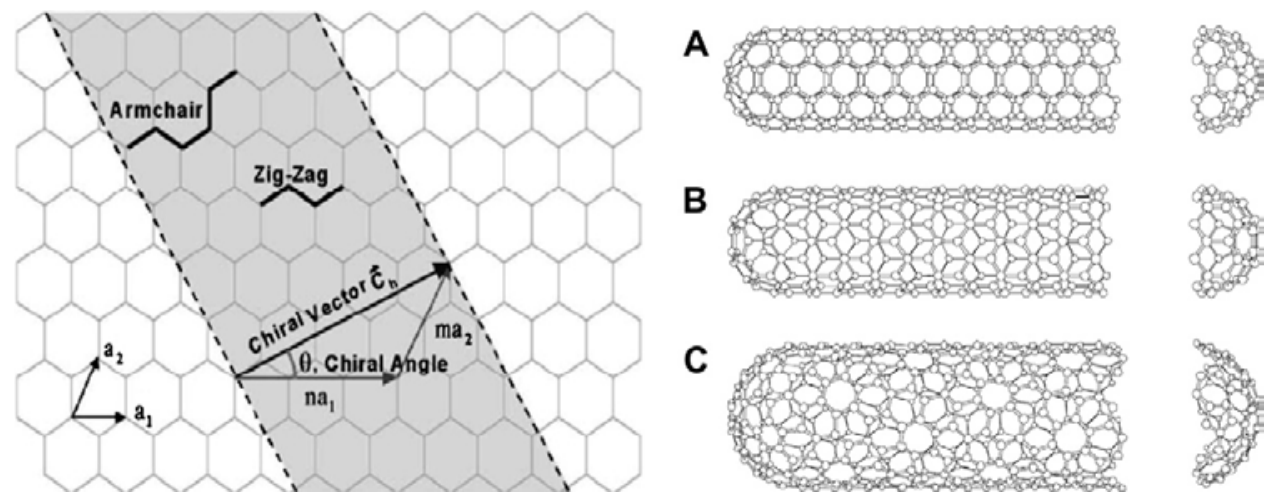

Figure 1.5. Schematic diagram showing how a hexagonal sheet of graphene is rolled to form a CNT with different chirality (A: armchair; B: zigzag; C: chiral). ${ }^{9,11-13}$

In the thesis, the properties of the SWCNT have been investigated. Depending on the "rolling angle" of the graphene sheet, SWCNT can be classified as armchair, zig-zag and chiral. The SWCNT chirality is defined by the chiral vector, $\mathrm{C}_{\mathrm{h}}=\mathrm{n} \mathbf{a}_{1}+\mathrm{ma}_{2}$ and is shown in Figure 1.5, where the integers $(n, m)$ are the number of steps along the unit vectors ( $\mathbf{a}_{1}$ and $\mathbf{a}_{2}$ ) of the hexagonal lattice structure for grapheme. ${ }^{9,12,13}$ By using the (n, m) naming scheme, the three types of chiralities of the carbon atoms around the nanotube circumference can be identified. If $n=m$, the nanotubes are called “armchair" CNT, as shown in Figure 1.6; while if $\mathrm{m}=0$, the nanotubes are called “zig-zag" CNT as shown in Figure 1.7; while any other remaining combinations are called "chiral" CNT as shown in Figure 1.8.

The structural configuration of $(5,5)$ armchair SWCNT are shown in Figure 1.6. The top left image in Figure 1.6 depicts the top view of the $(5,5)$ armchair SWCNT, and the other three images on the top side of Figure 1.6 depict the side view of a single unit-cell, double repeatition of an unit-cell, and 5 repeatitions of an unit-cell respectively. 
The 3 images at the bottom portion of Figure 1.6 depict the isometric view of the these configurations. The images show that armchair SWCNT have a crystal structure where every row and column in the SWCNT crystal are identical regardless of the number of repeating units while both ends of the SWCNT are devoid of unusual "arm" or points.

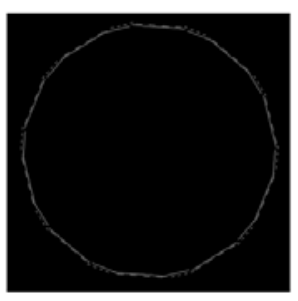

$>$ Armchair $(5,5)$ $>$ Diameter: $6.78 \AA$

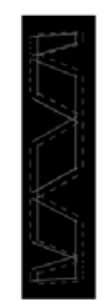

$\checkmark 1$ repeat unit $\checkmark$ Length: $2.46 \AA$

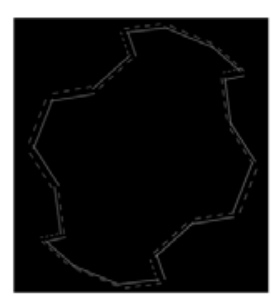

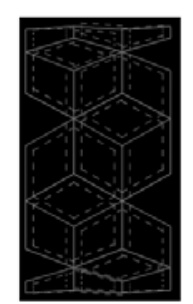

$\checkmark 2$ repeat unit

$\checkmark$ Length: $4.92 \AA$

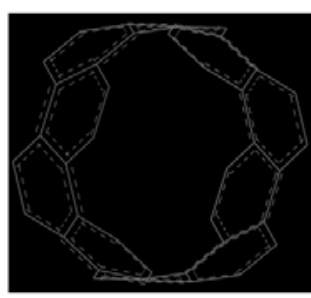

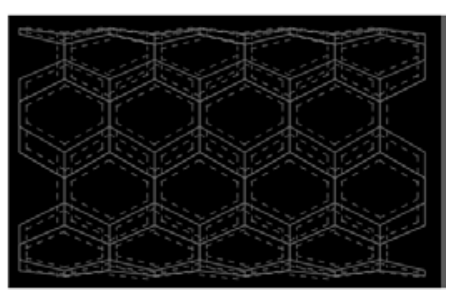

$\checkmark 5$ repeat unit $\checkmark$ Length: $12.30 \AA$

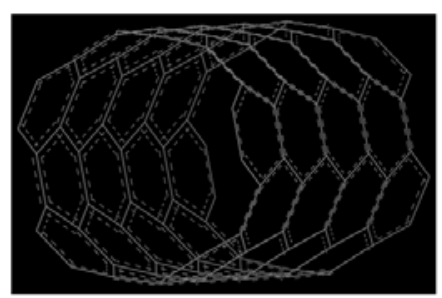

Figure 1.6 Images depicting various embodiments of an armchair SWCNT.

The structural configuration of $(9,0)$ zig-zag SWCNT is shown in Figure 1.7. The top left image in Figure 1.7 depicts the top view of the $(9,0)$ zig-zag SWCNT while the other three images on the top side of the Figure 1.7 depict the side view of a single unit-cell, double repeatition of an unit-cell, and 5 repeatitions of an unit-cell respectively. The 3 images at the bottom portion of Figure 1.7 depict the isometric view of the these configurations. Similar to the armchair configuration, the images show that zig-zag SWCNT have a crystal structure where every row and column in the SWCNT crystal are 
identical regardless of the number of repeating units while both ends of the SWCNT are devoid of unusual "arm" or points.

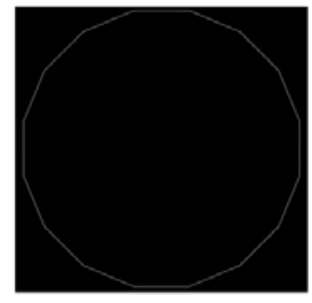

$>$ Zig zag $(9,0)$

>Diameter: $7.05 \AA$

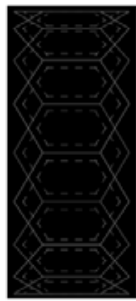

$\checkmark 1$ repeat unit $\checkmark$ Length: $4.26 \AA$

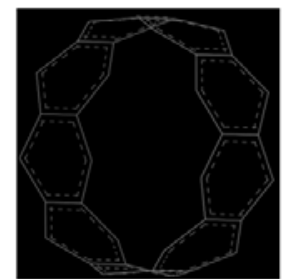

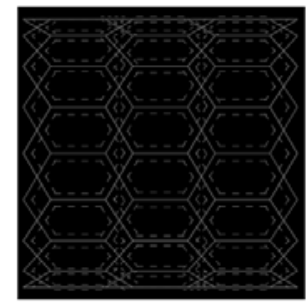

$\checkmark 2$ repeat unit $\checkmark$ Length: $8.52 \AA$

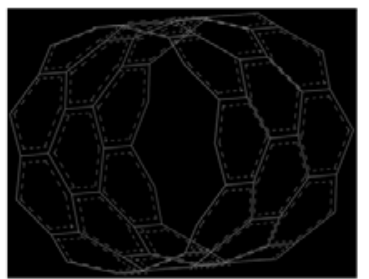

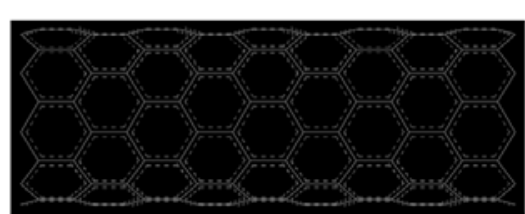

$\checkmark 5$ repeat unit

$\checkmark$ Length: $21.30 \AA$

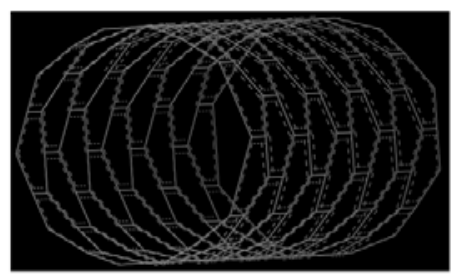

Figure 1.7 Images depicting various embodiments of a zig-zag SWCNT.

The structural configurations of the $(6,4)$ and $(6,5)$ chiral SWCNTare shown in Figure 1.8. The two images in the left side in Figure 1.8 show the top view of the two different chiral SWCNT, and the two images on the right in Figure 1.8 shows a single unit-cell of these two different configurations of chiral SWCNT. Figure 1.8 shows that the total length of a single unit-cell of chiral SWCNT is significantly different for the (6, 4) chiral SWCNT (which is only $18.57 \AA$ ) when compared to that of the $(6,5)$ SWCNT (which is $40.64 \AA$ ). However, when the crystal structure of the armchair and zig-zag SWCNT (as shown in Figure 1.6 and Figure 1.7), are compared with that of the chiral SWCNT significant differences are observed. Figure 1.8 shows that each successive row and column are staggerred and not aligned as in the case of armchair and zig-zag 
SWCNT., Both ends of the SWCNT are uneven due to staggerred placement of the hexagonal repeating units in the graphitic sheet.

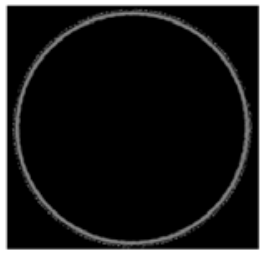

$>$ Chiral $(6,4)$

>Diameter: $6.83 \AA$

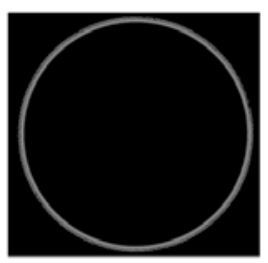

$>$ Chiral $(6,5)$

>Diameter: $7.47 \AA$

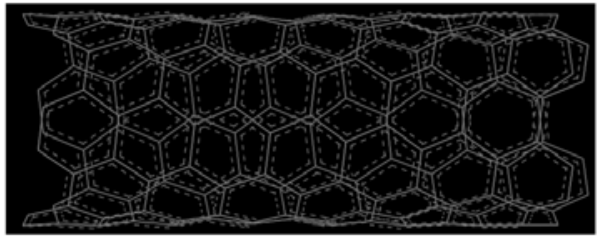

$\checkmark 1$ repeat unit

$\checkmark$ Length: $18.57 \AA$

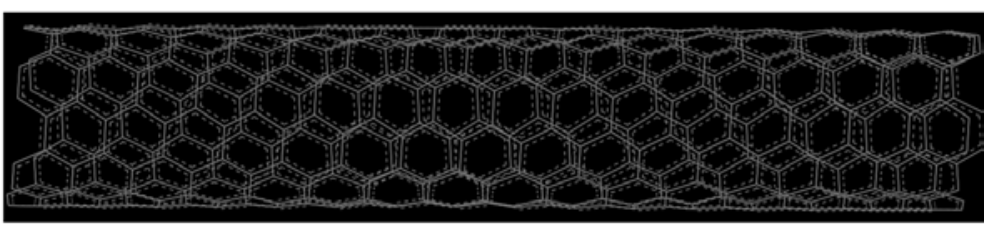

$\checkmark 1$ repeat unit

$\checkmark$ Length: $40.64 \AA$

Figure 1.8 Images depicting various embodiments of two different chiral SWCNT.

The chirality of the nanotube has a significant impact on the resultant material properties, such as electronic and thermo-physical properties. Since all of the possible configurations (i.e., the chirality) of a nanotube can be specified by the chiral vector (n, $\mathrm{m})$, the material properties can be segmented based on the specified by the chirality of each individual carbon nanotube It has been found and proved in previous research, if $(2 \mathrm{n}+\mathrm{m})$ is a multiple of 3 , then the carbon nanotube is considered to be "metallic CNT" (electrically conducting) while all other configurations of the carbon nanotube is classified as "semiconducting CNT". 9 Hence this confers a unique opportunity to obtain tunable materials properties by tuning the lattice structure (chirality) of individual SWCNT. ${ }^{11}$ 


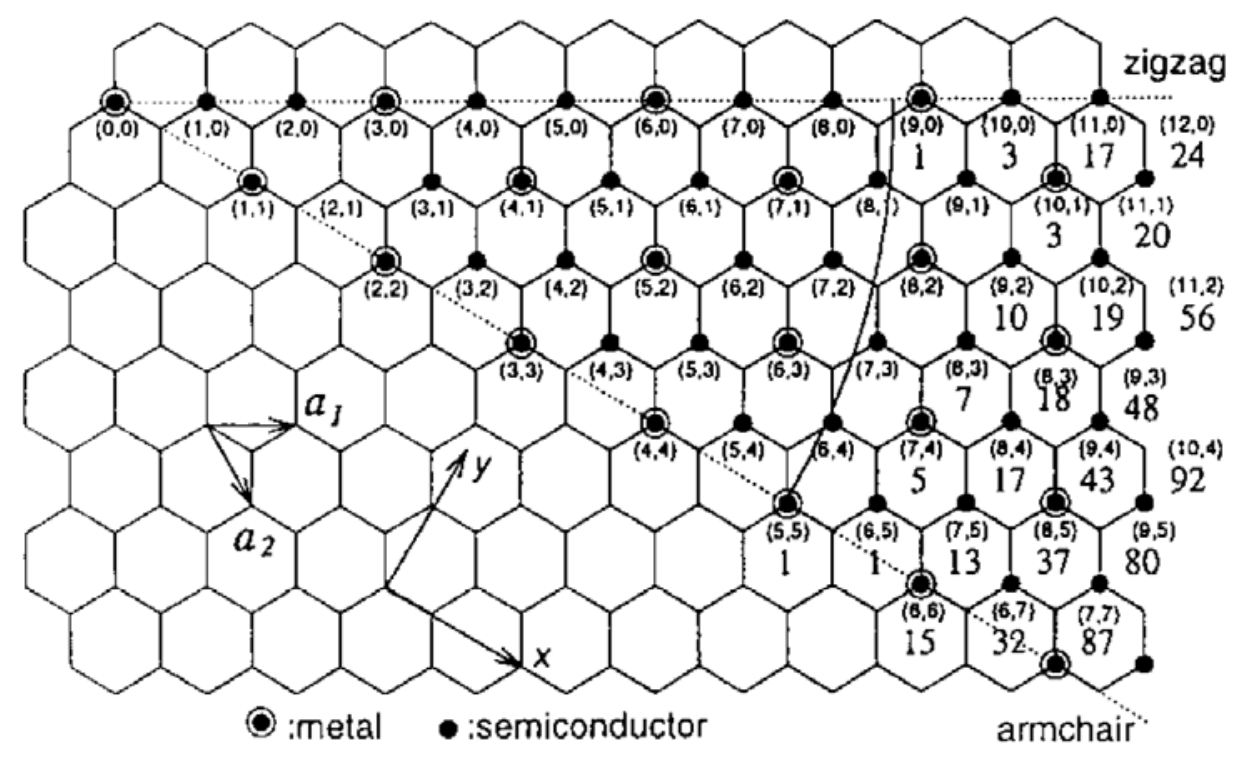

Figure 1.9 Schematic depicting the vector configuration that is used to specify the chirality of a carbon nanotube for $2 \mathrm{D}$ graphene sheet. The pairs of integers $(n, m)$ in the figure specify chiral vectors $\boldsymbol{C}_{\boldsymbol{h}}$ for carbon nanotubes, including zig-zag, armchair, and chiral tubules. Circled dots denote metallic tubules and the small dots are for semiconducting tubules. ${ }^{12}$

Based on the rule that distinguishes the metallic and semi-conducting CNT, it is observed that all the armchair CNT are metallic which corresponds to vector group that are inclined at $\sim 30^{\circ}$ orientation from the horizontal doted line (as shown in Figure 1.9). Considering the zig-zag CNT (which are specified with a chiral vector group corresponding to $\mathrm{m}=0$ ), as shown in Figure 1.9, these vector groups are represented by the horizontal dotted. Hence it can be estimated that approximately $1 / 3$ of the total set of zig-zag chiralities of CNT (i.e., chiralities corresponding to the horizontal dotted line) have metallic properties, while the other $2 / 3$ of the total set of zig-zag chiralities of CNT have the semi-conducting properties. This relationship is also apparent in Figure 1.9, 
where there are two sets of semi-conducting CNT between any two consecutive metallic CNT. Similar trends can be observed in the plot of Figure 1.9 for chiral CNT as well.
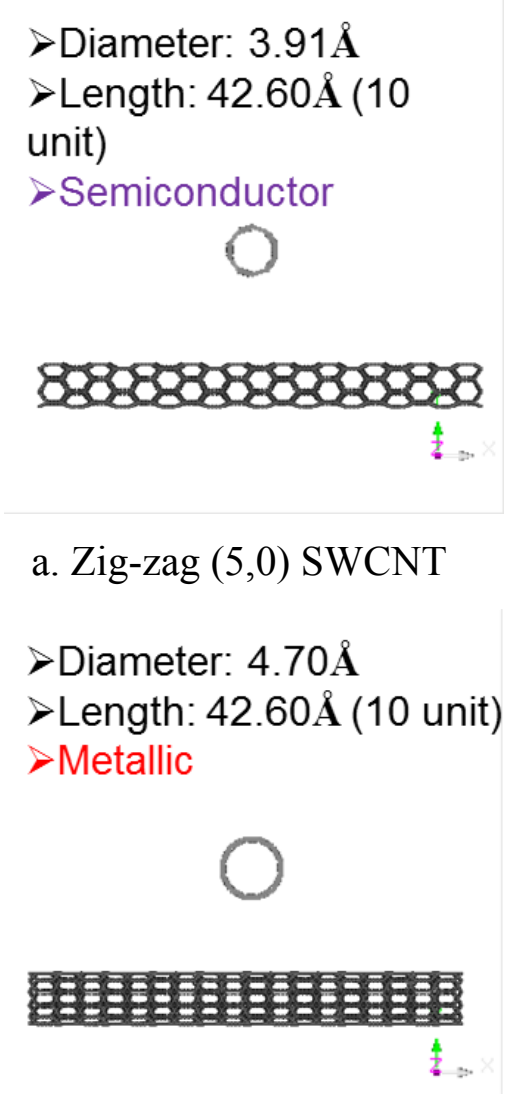
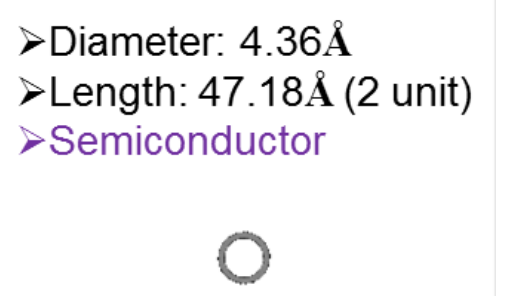

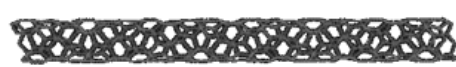

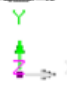

c. Chiral $(5,1)$ SWCNT

$>$ Diameter: $5.13 \AA$

$>$ Length: $55.87 \AA$ ( 2 unit)

$>$ Semiconductor
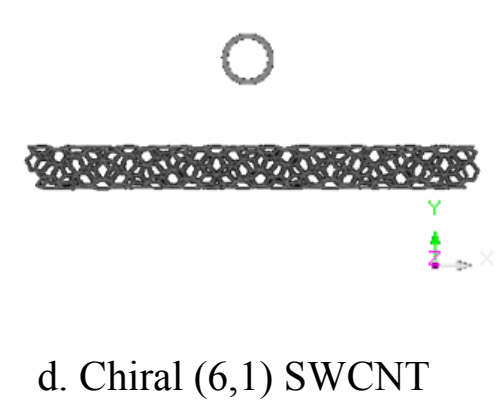

d. Chiral $(6,1)$ SWCNT
$>$ Diameter: $4.89 \AA$

$>$ Length: $53.15 \AA$ (2 unit)

$>$ Metallic

\section{0}

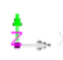

e. Chiral $(5,2)$ SWCNT

$>$ Diameter: $5.65 \AA$

$>$ Length: $30.72 \AA$ (2 unit)

$>$ Semiconductor

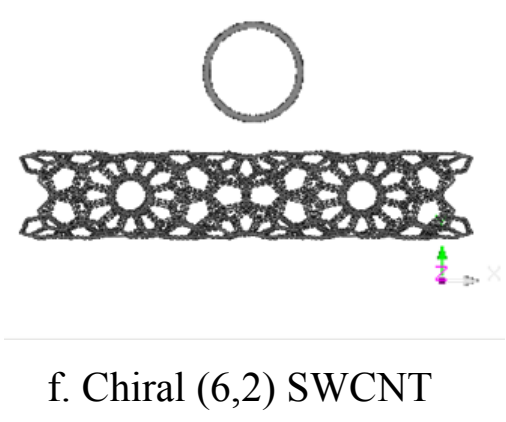

f. Chiral $(6,2)$ SWCNT

\section{b. Zig-zag $(6,0)$ SWCNT}

Figure 1.10 Comparison between the structures of SWCNT with different chirality. Each image is shown with different length scales and magnification (please refer to the listed information specifying the morphology of each SWCNT).

When the characteristics of the zig-zag carbon nanotube and the chiral carbon nanotubes are compared both are observed to have similar trends, especially for the frequency in the chirality distribution for nanotubes with metallic properties. Hence, a detailed comparison of the structure (such as diameter, total number of repeating units, total 
length, and the associated material properties) are shown in Figure 1.10, (it should be noted that the schematics are for reference only, since different length scales are used for each image, it is advisable to consider the numerical values for nanotube geometry that are specified for each schematic in the figure). From the side/left view of the carbon nanotube schematics in the figure, it is observed that the zig-zag carbon nanotube has a more orderly structure than the chiral carbon nanotube.

Stable colloidal solutions that are obtained from solvents doped with nanoparticles, usually at minute concentrations, are called "nanofluids". Typically the nanoparticles may be metallic (i.e., $\mathrm{Au}, \mathrm{Ag}, \mathrm{Ni}$, etc.), ceramic (i.e., $\mathrm{SiO}_{2}, \mathrm{TiO}_{2}$, etc.) or organic (i.e., carbon nanotube, fullerene $/ \mathrm{C}_{60}$, graphene sheets or platelets, etc.). Various types of solvents have been explored in the literature - such as water, oils, glycols, ionic liquids, etc. For high temperature applications (i.e., concentrated solar power/ CSP) molten salts have been demonstrated to enable stable nanofluids where the thermo-physical properties were enhanced on mixing with nanoparticles (the properties of the resulting nanofluids were found to be highly sensitive to small variations in the mixing procedure or the recipe for the synthesis of the nanofluids).

The enhanced material properties of the nanofluids that have been reported in the literature have led to speculation for their use in various applications, especially in heat transfer applications (i.e., thermal energy storage, cooling, thermal management, etc.). ${ }^{14}$ A nanoparticle is typically defined to have at least one characteristic dimension (i.e., 
diameter or length) that is less than $100 \mathrm{~nm}$. For achieving a stable colloidal nanoparticle dispersion in a solvent very low mass concentrations are recommended, typically $0.01 \%$ $\sim 1 \%$ and usually less than $5 \%$ percent (the lower limit typically applies to cylindrical or disk shaped nanoparticles and higher mass concentrations can be used for spherical nanoparticles). Initial studies in the nanofluids literature reported that the thermal conductivity and the convective heat transfer coefficient when compared to those of the pure solvent (i.e., base fluid itself). ${ }^{15}$ Hence, nanofluids are considered to be attractive materials for various applications, such as: electronic chip cooling, thermal management of high heat flux devices, fuel cells, cooling of engines, vehicle thermal management, etc.

The topics explored in this study are relevant for nanofluids and nanocomposites (nanoparticles mixed with solid materials and powders/ amorphous substances) as well as for heat transfer involving nanocoatings on heaters and heaters with nanostructured surfaces.

\subsection{Numerical computations}

Numerical computations (also called numerical analysis) are the study of algorithms that use numerical approximation for the problem of mathematical analysis. In recent decades, topics involving numerical computations are increasing progressively in various disciplines. One significant example is the discipline of computation fluid dynamics (CFD), which is a branch of fluid mechanics that leverages numerical methods 
to solve and analyze fluid flow problems. CFD techniques are also used in other allied topics in engineering, such as heat transfer and combustion (i.e., flow with chemical reactions or in other words: mass transfer equations, fluid flow equations and heat transfer/energy equations integrated with species transport equations). ${ }^{16-23}$ Typical digital computers are used for performing the calculations required in these simulations. CFD techniques have become powerful simulation technologies that are used in different problems, and CFD has been employed in solving various complex scenarios and problems in engineering. ${ }^{16,17,21,24}$

Numerical computations have enabled the solution of intractable problems by researchers. Apart from CFD, other examples where numerical computations have been used extensively to solve scientific and engineering problems as well as predict material and structural behavior at different length and time scales - include: stress and motion analysis by using finite element analysis (abbreviated as FEA) and molecular dynamics (abbreviated as MD).

\subsubsection{Molecular dynamics (MD) simulations}

Molecular dynamics simulations are typically used to simulate the temporal and spatial evolution of atoms in a thermodynamic system. ${ }^{2}$ The physical and chemical properties of various materials can be computed by using MD simulations. By assuming a particular model for the force fields arising from individual atoms, the position and motion of every atom in a system is solved by using the Newton's equation of motion at 
each time step. Therefore, by calculating the mutual interaction based on the assumed force field, the position and velocity can be estimated easily for each atom at each time step. The information obtained for the position and velocity of each atom, is key to calculating the material properties of the system.

Surface adsorption of fluid molecules on a nanoparticle surface, such as the interfacial layering between the carbon nanotube and the base fluid, is considered to be a unique feature which is responsible for enhancement of the thermal conductivity of nanofluids. In order to better understand the inter-molecular interactions between a carbon nanotube and the molecules of the fluid (base fluid or coolant fluid), molecular level models need to be formulated to describe the resultant transport mechanisms. However, it is cumbersome to perform experiments for observing the formation, dynamics and evolution of these adsorbed layers on the nanoparticle surface as well as determining their effect on the resulting material properties. The adsorbed layer is not easy to distinguish from the bulk phase (i.e., due to higher volumetric density of the adsorbed molecules, non-liner/non-Newtonian viscosity of the adsorbed phase compared to that in the bulk, etc.) and it is also difficult to measure the thickness of this adsorbed layer that forms a distinct, yet diffuse phase. ${ }^{3}$ The adsorbed layer can give rise to non-intuitive behavior - such as - impeding the transfer of thermal energy at the interface between the nanoparticle and the surrounding fluid molecules. This interfacial thermal resistance is called the Kapitza Resistance " $R_{k}$ ". Therefore, numerical computations were employed in this study to estimate the interactions between the various transport mechanisms that 
can modulate the values of the Kapitza Resistance. In this study, molecular dynamics was used to analyze and to estimate the values of interfacial thermal resistance (Kapitza Resistance, " $R_{k}$ "), as well as to estimate the thickness of the adsorbed liquid layer that forms on the surface of the carbon nanotube.

A number of studies in the literature have reported the estimated values of interfacial thermal resistance by using MD simulations. Shenogin et al. ${ }^{25}$ estimated that the interfacial thermal resistance between carbon nanotube and octane molecules is approximately $4.0 \times 10^{-8} \mathrm{~m}^{2} \cdot \mathrm{K} / \mathrm{W}$. Huxtable et al. ${ }^{26}$ calculated the interfacial thermal resistance for CNT in water solution to be $8.3 \times 10^{-8} \mathrm{~m}^{2} \cdot \mathrm{K} / \mathrm{W}$, where the carbon nanotube is modeled to be coated with a surfactant (sodium dodecyl sulfate, abbreviate as SDS). $\mathrm{Shin}^{2}$ reported that the interfacial thermal resistance for carbon nanotube and silica nanoparticle to be $3.99 \times 10^{-8} \mathrm{~m}^{2} \cdot \mathrm{K} / \mathrm{W} \sim 5.19 \times 10^{-8} \mathrm{~m}^{2} \cdot \mathrm{K} / \mathrm{W}$, and $4.58 \times 10^{-9} \mathrm{~m}^{2} \cdot \mathrm{K} / \mathrm{W} \sim$ $8.02 \times 10^{-9} \mathrm{~m}^{2} \cdot \mathrm{K} / \mathrm{W}$, respectively. $\mathrm{Jo}^{3}$ reported that the mean value of the interfacial thermal resistance to be $8.27 \times 10^{-8} \mathrm{~m}^{2} \cdot \mathrm{K} / \mathrm{W}$.

\subsection{Motivation of this study}

Extensive studies have been reported in the literature for estimating the values of interfacial thermal resistance " $R_{k}$ " at the interface between nanoparticles (such as carbon nanotubes) and various solvents. The previous studies have been restricted essentially to the armchair carbon nanotube, and primarily to a chirality value of $(5,5)$ carbon nanotube. The role of chirality of the carbon nanotube (such as armchair, chiral and 
zig-zag carbon nanotube) on the resulting values of interfacial thermal resistance " $R_{k}$ " have not yet been explored extensively in the literature. Hence the motivation of this study is to explore the impact of the chirality of a carbon nanotube (such as zig-zag and chiral carbon nanotube) as well as the size/geometry of a nanotube on the resulting values of the interfacial thermal resistance " $R_{k}$ ".

\subsection{Objective of this study}

The objective of this study is to investigate the effect of different chirality values on the interfacial thermal resistance of a single walled carbon nanotube (SWCNT). The estimation of interfacial thermal resistance " $R_{k}$ " can therefore help to identify the optimum carbon nanotube configuration for CSP/TES applications, as well as other energy conversion applications and experiments for studying phase-change phenomena on nanostructured heater surfaces. Also, the aim of this study is to predict the optimum size of nanoparticles with the goal of maximizing the thermal conductivity of nanofluids and nanocomposites.

\subsection{Hypothesis}

At solid-liquid interfaces, such as at the interface between a carbon nanotubes surrounded by water molecules, adsorption of the liquid molecules on the nanotube surface (or solid crystalline surface) leads to the formation of a semi-crystalline phase (which is also called the "compressed phase"). The thickness of the adsorbed layer has 
been measured in various experiments and is estimated to be $2 \sim 20 \mathrm{~nm}$, depending on the composition of the solid and fluid material.

Figure 1.11 shows an image obtained from a high resolution tunneling electron microscope (HRTEM) in experiments performed by Oh et al. ${ }^{27}$ The figure shows the formation of an adsorbed layer of molten liquid aluminum molecules on the surface of a sapphire crystal. The figure is obtained by overlaying the predictions from a numerical simulation on the image obtained by electron microscopy. The first layer of liquid atoms is shown schematically in Figure 1.11.

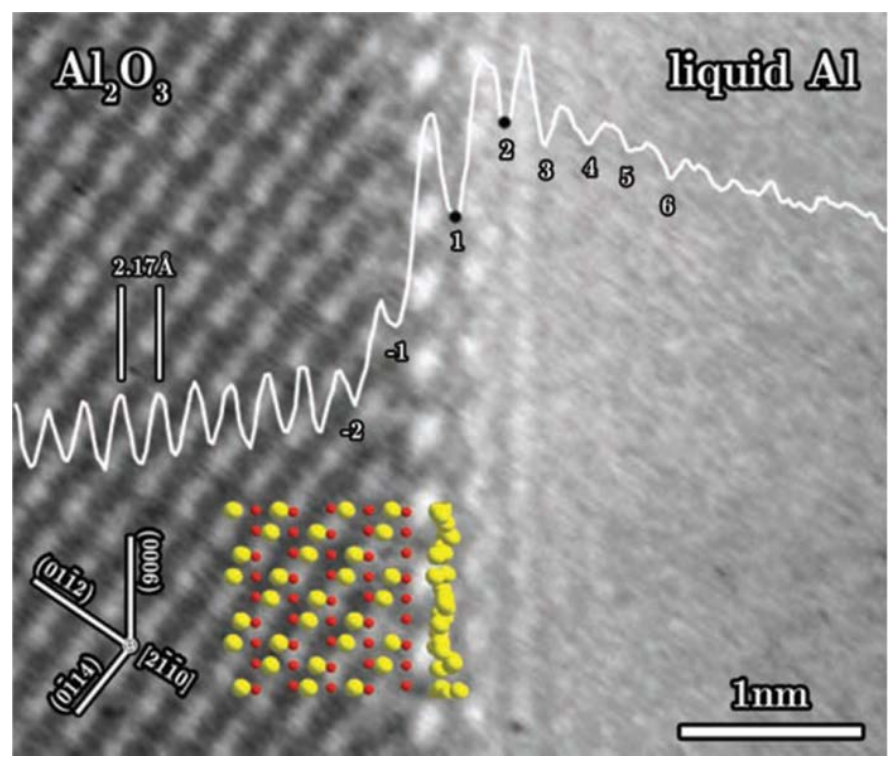

Figure 1.11 High resolution tunneling electron microscope (HRTEM) image of sapphire/liquid aluminum mixture. ${ }^{28}$

The predictions from the MD simulations show that the average mass density of this compressed phase is similar to that of the solid phase density of the fluid material. 
Various reports in the literature obtained from MD simulations have shown similar trends. Shin $^{2}$ reported that the compressed phase can modulate the thermo-physical properties of solvents mixed with nanoparticles. In another study, as shown in Figure 1.12 , for $n$-Tridecane molecules surrounding a carbon nanotube, compressed phase is formed by the fluid molecules that are adsorbed on the surface of the nanotube. In this figure, the two red circles represent the location of the density peaks (in terms of number of molecules per unit volume) in the compressed layer that were predicted by the results from the numerical simulations. The simulation results show that the peak value with the largest magnitude occurs on the surface of the nanotube.

The adsorbed layer of fluid molecules impedes thermal energy transport (i.e., interfacial thermal resistance) between the nanotube and fluid molecules in the bulk phase. The adsorbed layer of fluid molecules, owing to their higher values of molecular density (number of molecules per unit volume) are also expected to have different material properties - such as thermal conductivity, specific heat capacity, and rheological behavior. For example, the adsorbed layer is expected to display non-Newtonian rheology (i.e., shear thinning or shear thickening behavior) while the fluid molecules may have Newtonian behavior when pure (i.e., in the bulk phase and/ or in the absence of nanoparticles). The adsorbed layer can have higher specific heat capacity values than that of the bulk phase - thus serving in the role of a "thermal capacitance" or a "thermal battery" and can have a significant impact in thermal energy storage (TES) applications. In addition, the nanoparticles can induce chemical concentration gradients from the 
surface of the nanoparticle to the bulk phase owing to the preferential interaction of the surface atoms (on the nanotube) with the different chemical species of the fluid molecules. The resulting chemical concentration gradient (gradient of the chemical potential on the surface of the nanoparticle) can impede or facilitate the heat transfer to/ from the nanoparticle and the fluid molecules. Hence diodic behavior may be apparent for heat transfer from a hot nanoparticle to the surrounding colder fluid molecules (i.e., in one case the chemical concentration aids heat transfer - while on reversal of the temperature configuration, the chemical concentration can partially impede heat transfer). As a result, there may be higher levels of heat transfer depending on the direction of the temperature drop (i.e., either from hot solid to cold liquid or from hot liquid to cold solid). The combination of the interfacial thermal resistance and thermal capacitance arising from the adsorbed phase on the surface of the nanoparticle can be used in behavioral models as equivalent thermal impedance, especially for simulating unsteady conditions in the initial and boundary conditions. Such behavioral models can be useful for analyzing the material behavior in solar thermal energy storage applications, as well as for estimating the effective thermal impedance in thermal management/ cooling applications. The size of the nanoparticles can be effective in enhancing the resultant thermal conductivity of the mixture for a certain size range (say $\sim 50-100 \mathrm{~nm}$ ) while for other range of sizes the nanoparticles can be effective in enhancing the resultant specific heat capacity of the mixture (say $\sim 2-10 \mathrm{~nm}$ ). The optimum size range for each material property values are expected to depend on the material properties of the fluid phase and 
solid nanoparticles as well as on the size and morphology of the nanoparticles (i.e., chirality, composition, etc.).

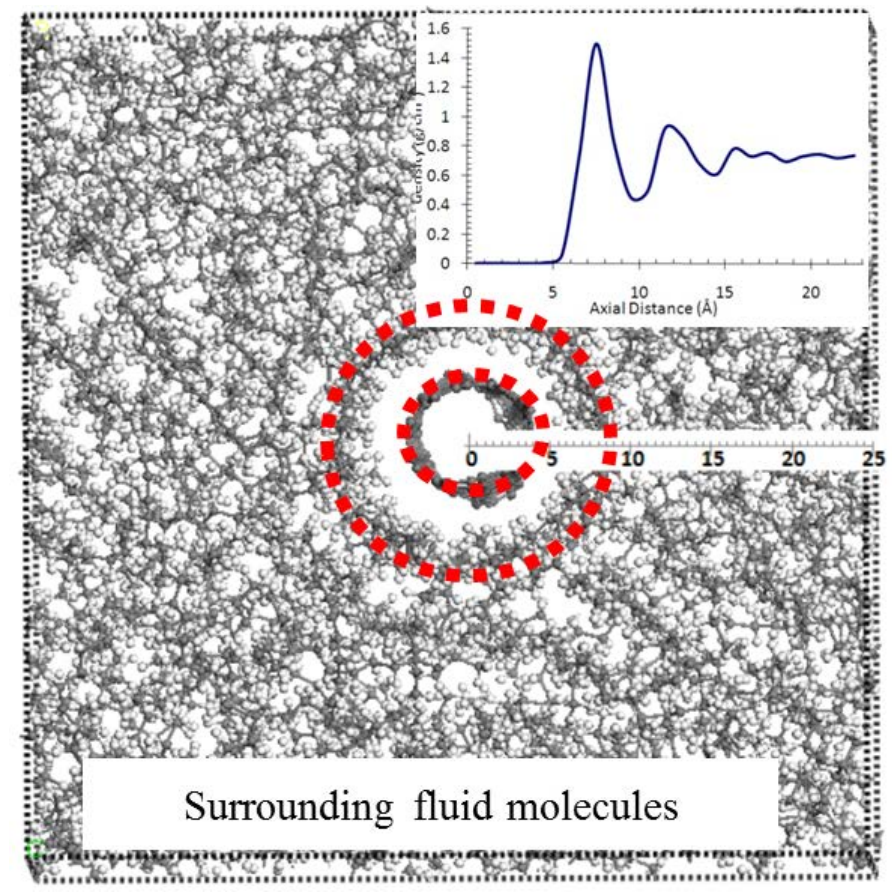

Figure 1.12 Equilibrium structure snapshot for n-Tridecane (scale in $\AA$ ).

\subsection{Significance of this study}

This study contributes to the thermal-fluid sciences and fundamental understanding of nano-scale transport phenomena by performing computational simulations that enable the prediction of the interfacial thermal resistance between a carbon nanotube and the fluid molecules for different values of chirality of the SWCNT that were implemented in the numerical models. The aim of this study is to explore the effect of carbon nanotube type (chirality) and carbon nanotube size on the interfacial thermal resistance. The results are obtained by performing MD simulations. 
Prior reports in the literature show that the properties of the solvent phase can be strongly affected by the values of interfacial thermal resistance " $R_{k}$ " that occurs between a nanoparticle (i.e., CNT) and the fluid molecules surrounding the nanoparticle. Hence the value of Kapitza resistance is important for estimating the critical size of the nanoparticles that will either maximize the value of net thermal conductivity of the nanofluids or the specific heat capacity of the mixture. However, the effect of the chirality of the nanoparticles (for a fixed composition of the molten salt) on thermo-fluidic properties (such as specific heat capacity) has not been explored extensively in the literature. Hence, in this numerical study the chirality of the carbon nanotube is varied parametrically and the values of interfacial thermal resistance " $R_{k}$ " are estimated for each chirality (such as armchair, chiral, and zigzag), using an approach similar to that reported by Jo et al. ${ }^{29}$ and the results from this study were used to estimate the value of critical dimensions of the nanoparticle for maximizing heat transfer (i.e., cooling) and thermal energy storage. 


\section{SECTION 2. LITERATURE REVIEW}

The literature is replete with experimental reports demonstrating significant enhancement of heat flux during phase change on heaters with on nanostructured surfaces when compared to that of a plain surface. The enhancement of boiling heat flux caused by a carbon nanotube coating was reported to be $60-120 \%{ }^{30-32}$ The material properties of nanofluids and nanocomposites, such as mechanical, optical, electrical and thermal properties, are reported to be enhanced significantly. ${ }^{26,33}$ The parameters affecting the resultant material properties of nanolfuid and nanocomposite materials include the aspect ratio of nanoparticles, volume faction of nanoparticles (or mass fraction), nature of the inter-molecular interactions (i.e., van Der Waals, ionic, etc.) between nanoparticle and the solvent molecules, etc. ${ }^{25}$ These parameters also affect the transport properties and the resulting transport phenomena for these mixtures. ${ }^{25}$

Compared to the planar (platelet) shape of graphite and graphenes, the rolled-up tube shaped structure of carbon nanotubes causes the thermal and electrical conductivity of the single wall carbon nanotubes to be modulated. ${ }^{34}$ The thermal conductivity of carbon nanotubes are estimated to be as high as 20 to 100 times compared to that of copper. ${ }^{35}$ The percolation threshold is typically very low $(\sim 1 \%$ or less $)$ due to the extremely high value of aspect ratio of carbon nanotubes. ${ }^{36}$ Therefore, theoretically 
speaking, at mass concentration well above the percolation threshold, a high value of thermal conductivity can be obtained for composites obtained by doping with carbon nanotube. In addition, the experimental data has been reported to be significantly smaller that the theoretically estimated values for the thermal conductivity values of these nanocomposites. The interfacial thermal resistance, also known as Kapitza Resistance (" $R_{k}$ "), is expected to be the dominant parameter which modulates the thermal properties of the nanomaterial, and is believed to be responsible for the lower values of thermal conductivity measured for these mixtures (compared to the prediction from the numerical models based on percolation theory). Kapitza Resistance impedes thermal energy transfer (and therefore causes an abrupt temperature change) at the interface between two materials, and therefore it is also called as the "interfacial thermal resistance". Kapitza Resistance occurs due to the impedance mismatch of the vibration frequency of the components in the mixture, which results in scattering of phonons at the material interfaces. This arises from the molecular interactions at the material interfaces, such as due to weak Van der Waals forces and ionic forces between the nanoparticles and the solvent/ matrix materials as well as stearic hindrances/interactions of the solvent molecules. ${ }^{33}$

\subsection{Prior investigations at the multi-phase flows and heat transfer laboratory}

Prior research activities at the Multi-Phase Flows and Heat Transfer Laboratory (MPFHTL) at Texas A\&M University focused on topics relating to nanofluids and nanocoatings for modulating the thermo-physical properties and transport phenomena in 
multi-phase flows. A brief summary of prior research activities on these topics and their relation to the current study will be discussed next.

In prior studies the effect of heaters with nanocoatings and heaters with nanostructured surfaces were explored for enhancing the boiling heat flux, both for pool boiling and flow boiling systems. Also, carbon nanotube coatings were observed to enhance the heat flux in compact condensers. These studies have significant implications for improving the energy efficiency of Heating Ventilations and Air Condition (HVAC) systems as well as for electronic chip cooling applications. Other applications of these thermal management platforms range from solar thermal to laser diodes (for manufacturing applications). In various applications (such as cooling of laser diodes used for manufacturing applications) the thermal load on the cooling system can exceed $\sim 100 \mathrm{~kW} / \mathrm{cm}^{2}$ with the requirement for temperature fluctuations to be limited to less than $1 \mathrm{~K}$. Hence for these applications the heat transfer coefficient required for the cooling system is expected to exceed $\sim 100 \mathrm{~kW} / \mathrm{cm}^{2} \cdot \mathrm{K}$. Boiling is the only cooling technique that can enable heat fluxes in this range. Hence, boiling heat transfer enhancement is key to the development of several cutting edge technologies. Surface modifications involving nanoparticle coatings and nanostructured heater surfaces have been demonstrated to achieve significant enhancement in boiling heat flux.

It has been observed that for both nanofluids and nanocoatings, the magnitudes of heat transfer enhancement are subliminally affected by the thermal impedance network. In 
this network, Kapitza Resistance, which appears at the interface between various material components in the mixture, is a dominant parameter. The thermal impedance phenomena can also anomalously affect the specific heat capacity and the viscosity of the nanofluids in addition to the thermal conductivity values. , $3,28,37^{-13}$

A significant enhancement of phase change heat transfer was observed during boiling (both pool boiling and flow boiling) on heaters with nanostructured surfaces or surface nanocoatings - compared to that on plain heaters (i.e., heaters without nanocoatings or nanostructures). ${ }^{30,31,38-41}$ In these studies, the significant enhancement of heat flux values were observed during pool boiling of refrigerants on a heater coated with carbon nanotube coating (when compared with flat heater surfaces), The enhancements were observed for both nucleate and file boiling regimes as well as for critical heat flux (CHF) values. Surprisingly, even though carbon nanotube has higher thermal conductivity than silicon, higher levels of enhancement of pool boiling heat flux (especially critical heat flux) was observed for heaters with silicon nanofins. ${ }^{31}$

Subsequently, Singh et $\mathrm{al}^{33}$, Unnikrishnan et $\mathrm{al}^{42}$, and $\mathrm{Singh}^{43}$, and showed that the Kapitza Resistance (" $R_{k}$ ") for carbon nanotubes at the interface with the working fluid is 1000 times higher than that for the silicon nanofin structures. ${ }^{33,42,43}$ Therefore, Kapitza Resistance was identified as the dominant parameter for determining the level of heat flux enhancement for the nanocoatings. Hence, it is not surprising that the silicon 
nanofins yield higher values of critical heat flux than carbon nanotubes, despite the fact that carbon has a higher thermal conductivity than silicon.

A number of significant studies have been reported to explore the thermo-physical properties of nanomaterial, i.e. nanofluids. Nelson et al. ${ }^{44}$ reported up to $50 \%$ in the enhancement of the value of the specific heat capacity for poly-alpha-olefin oil, usually abbreviated as PAO, when mixed with graphite nanoparticles at a mass concentration of $0.6 \%$. Shin and Banerjee ${ }^{45-47}$ reported that the specific heat capacity of molten salt eutectics was enhanced by $\sim 20-120 \%$ on doping with silica nanoparticles at a mass concentration of $1 \%$. Jo and Banerjee ${ }^{48}$ reported that the specific heat capacity of molten salts was enhanced significantly in both solid and liquid phase on doping with organic nanoparticles. Additionally, a diverse range of results in the literature were reported to study the enhancement of specific heat capacity of various solvents when mixed with nanoparticles. ${ }^{2,28,48-55}$ Similar studies involving viscosity measurements of nanofluids were also reported in the literature. ${ }^{56,57}$

Also, a preliminary analytical model was developed which includes the contribution to the internal energy from the various components and phases of nanofluid suspensions. $^{46,58,59}$ Contributions to the total internal energy from the "compressed phase" (which is the layer of solvent molecules adsorbed on the surface of the nanoparticles, also called as the "ICE layer") was accounted for - in this model. 
$\mathrm{Yu}$ et $\mathrm{al}^{60}$ performed a study to investigate the efficacy of aqueous nanofluids in enhancing the forced convective heat transfer during internal flow in micro-channels. In this study, a simple analytical model for the specific heat capacity of nanoparticle suspensions in a solvent was explored. The effect of the mass concentration of nanoparticles, temperature, and imposed shear rate on the rheological properties of nanofluids was discussed in this report. By using an array of temperature nanosensors or "Thin Film Thermocouples (TFT)", the axial temperature gradient in the flow direction was measured successfully. Convective heat transfer values were derived from the experiments by using the wall temperature gradient measurements that were obtained in these experiments. ${ }^{38-40,61-63}$ In these studies, the nanoparticle material, concentration of the nanoparticles, duration of experimentation, flow rates and surface temperature were reported to affect the thermal performance of the nanofluids.

The effect of different parameters, i.e. chemistry, polymer chains, isomers and mixtures, was explored by Singh et al. $^{33}$ by using MD simulations. The Large-scale Atomic/Molecular Massively Parallel Simulator (LAMMPS) was used to perform the MD simulations and to estimate the values of interfacial thermal resistance. The simulation results show that the interfacial thermal resistance values are strongly sensitive to the chemical composition of the fluids. To study the effect of chemistry on the interfacial thermal resistance, MD simulations were performed using various solvent material properties, such as water, ethyl alcohol and 1- hexane. The simulations showed that the interfacial thermal resistance between a $(5,5)$ single wall carbon 
nanotube and the solvents were $2.13 \times 10^{-8}, 4.74 \times 10^{-8}$, and $7.29 \times 10^{-8} \mathrm{~m}^{2} \cdot \mathrm{K} / \mathrm{W}$ for water, ethyl-alcohol and 1-hexane, respectively. Also it was reported by Singh et al. that the length of the carbon nanotube can affect the estimated values of the interfacial thermal resistance, i.e. the value of interfacial thermal resistance fluctuates when using the small carbon nanotubes, but becomes steady with a larger length of carbon nanotube. It was reported in this study that regardless of the chemical composition, the interfacial thermal resistance also depends on the molecular structure and varies significantly with variation on the length of the polymer chains, as well as their isomers and mixtures of the same material (consisting of different chemical structures - i.e., polymers with different lengths and isomers).

Numerical simulations, i.e. MD simulations, were also performed to complement the results obtained from these experiments. The estimates for the values of interfacial thermal resistance, also called as Kapitza Resistance, for both organic and inorganic (ceramic) nanoparticles were investigated by using MD simulations. The simulations were performed for different solvent compositions (i.e., aqueous coolants, organic coolants and inorganic coolants such as binary molten salt eutectics). ${ }^{29,33,43}$

$$
\begin{array}{ll}
d_{c}=2 * k_{f} * R_{k} & \text { Equation } 1
\end{array}
$$

Estimated value of the interfacial thermal resistance " $R_{k}$ " between a given nanoparticle and the solvent can be used to estimate the critical particle diameter, also called the 
"optimum size" $\left(d_{c}\right)$. For example, for a spherical nanoparticle, the optimum size is expected to maximize the thermal conductivity of the mixtures. The critical particle diameter (that maximizes the thermal conductivity of the mixture) can be calculated using the Equation 1, where the $k_{f}$ is the thermal conductivity of the solvent phase.

Therefore, the prior knowledge of the range of the values for the interfacial thermal resistance (" $R_{k}$ ") is essential for the proper design of experiments, such as for the study of transport phenomena involving nanofluids as well as for the study of phase change phenomena involving heaters with nanostructured surfaces.

The interfacial thermal resistance has been investigated extensively, both using experiment and numerical techniques in last decade. It has been found that interfacial thermal resistance depends on the material properties of the surrounding molecules of the fluid as well as that of the nanoparticles. Based on the large volume of studies reported in the literature, large variations exist in the estimated values of the interfacial thermal resistance. The estimated values for interfacial thermal resistance vary from $0.76 \times 10^{-8} \mathrm{~m}^{2} \cdot \mathrm{K} / \mathrm{W}$ to $20 \times 10^{-8} \mathrm{~m}^{2} \cdot \mathrm{K} / \mathrm{W}$ for $\mathrm{CNT}$ mixed with various matrix (solvent) materials, as reported in the literature.

In-house code called "Macromolecular Reality MD (MRMD)" was used by Shenogin et al. ${ }^{25}$ to conduct a series of molecular dynamics simulations for armchair carbon nanotubes that were immersed in octane. Two groups of numerical models were used 
to estimate the values for Kapitza Resistance (" $R_{k}$ "). One numerical model consisting of 100 atoms in an individual single wall carbon nanotube crystal with a total length of $12.22 \AA$ predicted a value of $14.7 \times 10^{-8} \mathrm{~m} \cdot{ }^{2} \mathrm{~K} / \mathrm{W}$ for the Kapitza Resistance value. The other numerical model predicted a value of $4.52 \times 10^{-8} \mathrm{~m}^{2} \cdot \mathrm{K} / \mathrm{W}$ for Kapitza resistance, considering a system of 720 atoms in an individual single wall carbon nanotube crystal with a total length of $86.96 \AA$. All of these simulations were restricted to a fixed value of the chirality, typically $(5,5)$ carbon nanotube. However, it is expected that the chirality of the carbon nanotube will also affect the value of Kapitza resistance for the same solvent. This is the motivation of this study, i.e., to explore the variation of Kapitza resistance with variation in the chirality of a carbon nanotube for a given solvent. 


\section{SECTION 3. NUMERICAL PROCEDURE}

\subsection{Carbon nanotube and molten salt used in numerical computations}

Carbonate salt eutectic $\left(\mathrm{Li}_{2} \mathrm{CO}_{3}-\mathrm{K}_{2} \mathrm{CO}_{3}\right)$ with $62: 38$ molar ratio, which has a melting point of $488{ }^{\circ} \mathrm{C}$, This composition of carbonate salt eutectic was chosen for this study due to the good thermal stability of this material in liquid phase - which makes it compatible for high temperature applications in TES and CSP. Armchair SWCNT, Chiral SWCNT, and Zigzag SWCNT were chosen for the simulations performed in this study. Figure 3.1 shows the molecular structure of the carbonate salts used in this study. Figure 3.2 illustrates the lattice structure of each type of carbon nanotube used in this study.
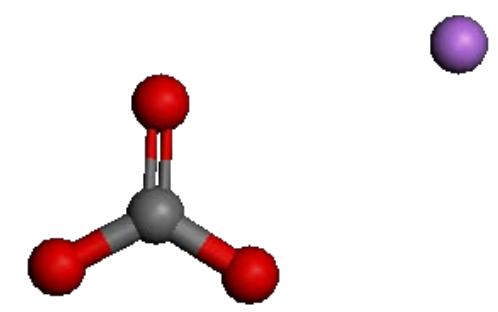

a. $\mathrm{K}_{2} \mathrm{CO}_{3}$ molecules in Materials Studio
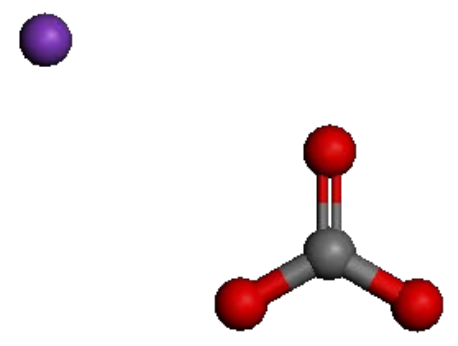

b. $\mathrm{Li}_{2} \mathrm{CO}_{3}$ molecules in Materials Studio

Figure 3.1 Schematic of the base liquid molecules. 

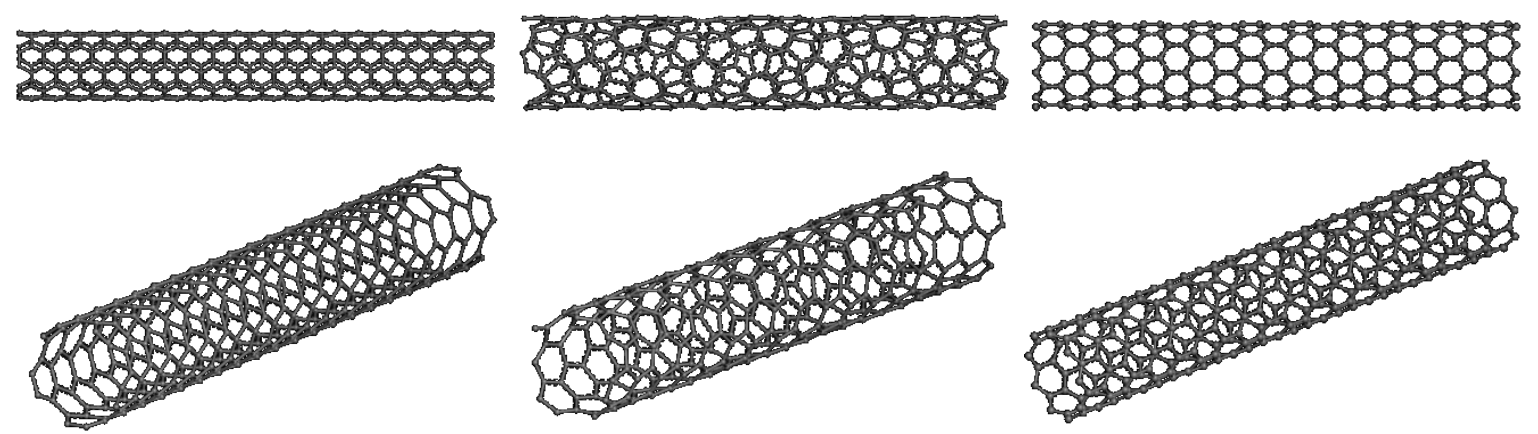

a. $(5,5)$ Armchair SWCNT

b. $(7,3)$ Chrial SWCNT

c. $(9,0)$ Zig-zag SWCNT

Figure 3.2. Schematic of carbon nanotube crystal structure with different chirality values used in this study. ${ }^{11}$

In order to investigate the effects of CNT type and size on the interfacial thermal resistance values, the values for the diameter of the SWCNT were varied from $6.78 \AA$ to $8.14 \AA$ for armchair SWCNT, from $4.36 \AA$ to $8.72 \AA$ for chiral SWCNT, and from 3.91 $\AA$ to $7.83 \AA$ for zig-zag SWCNT. All the parameters, such as diameter, length, property values and the total number of atoms for each type of SWCNT are listed in Table 3.1 to Table 3.7. These values were used in the numerical simulations performed in this study.

Table 3.1. Parameters for Armchair SWCNT

\begin{tabular}{|c|c|c|c|c|c|c|c|}
\hline \multicolumn{7}{|c|}{ Armchair SWCNT } \\
\hline No. & CNT & Property & $\begin{array}{c}\text { No. of } \\
\text { atoms in } \\
\text { the } \\
\text { nanotubes }\end{array}$ & $\begin{array}{c}\text { Diameter } \\
(\AA)\end{array}$ & $\begin{array}{c}\text { Nanotube } \\
\text { length } \\
(\AA)\end{array}$ & $\begin{array}{c}\text { No. of } \\
\text { periodic } \\
\text { nanotube }\end{array}$ & $\begin{array}{c}\text { No. of } \\
\text { eutectic } \\
\text { molecules }\end{array}$ \\
\hline 1 & $(5,5)$ & Metallic & 400 & 6.78 & 49.19 & 20 & 9000 \\
\hline 2 & $(6,6)$ & Metallic & 480 & 8.14 & & & \\
\hline
\end{tabular}


Table 3.2. Parameters for Zig-zag SWCNT

\begin{tabular}{|c|c|c|c|c|c|c|c|}
\hline \multicolumn{8}{|c|}{ Zig-zag SWCNT } \\
\hline No. & CNT & Property & $\begin{array}{c}\text { No. of } \\
\text { atoms in } \\
\text { the } \\
\text { nanotubes }\end{array}$ & $\begin{array}{c}\text { Diameter } \\
(\AA ̊)\end{array}$ & $\begin{array}{c}\text { Nanotube } \\
\text { length } \\
(\AA)\end{array}$ & $\begin{array}{c}\text { No. of } \\
\text { periodic } \\
\text { nanotube }\end{array}$ & $\begin{array}{c}\text { No. of } \\
\text { eutectic } \\
\text { molecules }\end{array}$ \\
\hline 1 & $(5,0)$ & S-C & 200 & 3.91 & \multirow{6}{*}{42.60} & \multirow{6}{*}{10} & \multirow{6}{*}{9000} \\
\hline 2 & $(6,0)$ & Metallic & 240 & 4.70 & & & \\
\hline 3 & $(7,0)$ & S-C & 280 & 5.48 & & & \\
\hline 4 & $(8,0)$ & S-C & 320 & 6.26 & & & \\
\hline 5 & $(9,0)$ & Metallic & 360 & 7.05 & & & \\
\hline 6 & $(10,0)$ & S-C & 400 & 7.83 & & & \\
\hline
\end{tabular}

Table 3.3. Parameters for Chiral (n, 1) SWCNT

\begin{tabular}{|c|c|c|c|c|c|c|c|}
\hline \multicolumn{8}{|c|}{ Chiral $(\mathrm{n}, 1)$ SWCNT } \\
\hline No. & CNT & Property & $\begin{array}{c}\text { No. of } \\
\text { atoms in } \\
\text { the } \\
\text { nanotubes }\end{array}$ & $\begin{array}{c}\text { Diameter } \\
(\AA)\end{array}$ & $\begin{array}{c}\text { Nanotube } \\
\text { length } \\
(\AA)\end{array}$ & $\begin{array}{c}\text { No. of } \\
\text { periodic } \\
\text { nanotube }\end{array}$ & $\begin{array}{c}\text { No. of } \\
\text { eutectic } \\
\text { molecules }\end{array}$ \\
\hline 1 & $(5,1)$ & $\mathrm{S}-\mathrm{C}$ & 248 & 4.36 & 47.44 & 2 & \multirow{6}{*}{9000} \\
\hline 2 & $(6,1)$ & $\mathrm{S}-\mathrm{C}$ & 344 & 5.13 & 55.87 & 2 & \\
\hline 3 & $(7,1)$ & Metallic & 228 & 5.91 & 32.16 & 1 & \\
\hline 4 & $(8,1)$ & S-C & 292 & 6.69 & 36.40 & 1 & \\
\hline 5 & $(9,1)$ & S-C & 728 & 7.47 & 81.28 & 2 & \\
\hline 6 & $(10,1)$ & Metallic & 888 & 8.25 & 89.76 & 2 & \\
\hline
\end{tabular}


Table 3.4. Parameters for Chiral (n, 2) SWCNT

\begin{tabular}{|c|c|c|c|c|c|c|c|}
\hline \multicolumn{8}{|c|}{ Chiral $(\mathrm{n}, 2)$ SWCNT } \\
\hline No. & CNT & Property & $\begin{array}{c}\text { No. of } \\
\text { atoms in } \\
\text { the } \\
\text { nanotubes }\end{array}$ & $\begin{array}{c}\text { Diameter } \\
(\AA ̊)\end{array}$ & $\begin{array}{c}\text { Nanotube } \\
\text { length } \\
(\AA)\end{array}$ & $\begin{array}{c}\text { No. of } \\
\text { periodic } \\
\text { nanotube }\end{array}$ & $\begin{array}{c}\text { No. of } \\
\text { eutectic } \\
\text { molecules }\end{array}$ \\
\hline 1 & $(5,2)$ & Metallic & 312 & 4.89 & 53.81 & 2 & \multirow{6}{*}{9000} \\
\hline 2 & $(6,2)$ & S-C & 208 & 5.65 & 30.72 & 2 & \\
\hline 3 & $(7,2)$ & S-C & 536 & 6.41 & 69.74 & 2 & \\
\hline 4 & $(8,2)$ & Metallic & 336 & 7.18 & 38.57 & 2 & \\
\hline 5 & $(9,2)$ & S-C & 824 & 7.95 & 86.47 & 2 & \\
\hline 6 & $(10,2)$ & S-C & 496 & 8.72 & 47.44 & 2 & \\
\hline
\end{tabular}

Table 3.5. Parameters for Chiral (n, 3) SWCNT

\begin{tabular}{|c|c|c|c|c|c|c|c|}
\hline \multicolumn{7}{|c|}{ Chiral (n, 3) SWCNT } \\
\cline { 1 - 6 } No. & CNT & Property & $\begin{array}{c}\text { No. of } \\
\text { atoms in } \\
\text { the } \\
\text { nanotubes }\end{array}$ & $\begin{array}{c}\text { Diameter } \\
(\AA)\end{array}$ & $\begin{array}{c}\text { Nanotube } \\
\text { length } \\
(\AA)\end{array}$ & $\begin{array}{c}\text { No. of } \\
\text { periodic } \\
\text { nanotube }\end{array}$ & $\begin{array}{c}\text { No. of } \\
\text { eutectic } \\
\text { molecules }\end{array}$ \\
\hline 1 & $(7,3)$ & S-C & 632 & 6.96 & 75.73 & 2 & \multirow{2}{*}{9000} \\
\hline 2 & $(8,3)$ & S-C & 776 & 7.71 & 83.91 & 2 & \\
\hline 3 & $(9,3)$ & Metallic & 468 & 8.47 & 46.08 & 3 & \\
\hline
\end{tabular}


Table 3.6. Parameters for Chiral (n, 4) SWCNT

\begin{tabular}{|c|c|c|c|c|c|c|c|}
\hline \multicolumn{7}{|c|}{ Chiral (n, 4) SWCNT } \\
\cline { 1 - 6 } No. & CNT & Property & $\begin{array}{c}\text { No. of } \\
\text { atoms in } \\
\text { the } \\
\text { nanotubes }\end{array}$ & $\begin{array}{c}\text { Diameter } \\
(\AA)\end{array}$ & $\begin{array}{c}\text { Nanotube } \\
\text { length } \\
(\AA)\end{array}$ & $\begin{array}{c}\text { No. of } \\
\text { periodic } \\
\text { nanotube }\end{array}$ & $\begin{array}{c}\text { No. of } \\
\text { eutectic } \\
\text { molecules }\end{array}$ \\
\hline 1 & $(6,4)$ & S-C & 456 & 6.83 & 55.71 & 3 & \multirow{2}{*}{9000} \\
\hline 2 & $(7,4)$ & Metallic & 744 & 7.55 & 82.16 & 2 & \\
\hline 3 & $(8,4)$ & S-C & 448 & 8.29 & 45.08 & 4 & \\
\hline
\end{tabular}

Table 3.7. Values of parameters used in the Molecular dynamics (MD) simulations

\begin{tabular}{|c|c|c|c|c|c|c|c|c|c|c|c|}
\hline \multicolumn{5}{|c|}{ Lennard-Jones constants } & \multicolumn{2}{|c|}{ Stretching } & \multicolumn{2}{|c|}{ Bending } & \multicolumn{3}{|c|}{ Torsion } \\
\hline Materials & Interaction & $\begin{array}{c}\varepsilon \\
\text { (kcal/ } \\
\text { mol) }\end{array}$ & $\begin{array}{c}\sigma \\
(\AA)\end{array}$ & Charge & $\mathrm{K}_{\mathrm{s}}$ & $r_{0}$ & $\mathrm{~K}_{\mathrm{b}}$ & $\theta_{0}$ & $\mathrm{~K}_{\mathrm{t}}$ & d & $\mathrm{N}$ \\
\hline SWCNT & $\mathrm{C}-\mathrm{C}$ & 0.105 & 3.851 & 0 & 480 & 1.34 & 90 & 120 & $\begin{array}{c}12.37 \\
00\end{array}$ & -1.00 & 2 \\
\hline \multirow{4}{*}{ Eutectic } & $\mathrm{C}-\mathrm{C}$ & 0.148 & 3.617 & +1.54 & \multirow{4}{*}{4612} & \multirow{4}{*}{1.29} & \multirow{4}{*}{$\begin{array}{c}107.43 \\
54\end{array}$} & \multirow{4}{*}{120} & \multirow{4}{*}{$\begin{array}{c}1.139 \\
2\end{array}$} & \multirow{4}{*}{-1.00} & \multirow{4}{*}{0} \\
\hline & $\mathrm{O}-\mathrm{O}$ & 0.228 & 2.880 & -1.18 & & & & & & & \\
\hline & $\mathrm{Li}-\mathrm{Li}$ & 4.735 & 2.839 & +1.00 & & & & & & & \\
\hline & $\mathrm{K}-\mathrm{K}$ & 5.451 & 3.197 & +1.00 & & & & & & & \\
\hline
\end{tabular}

\subsection{Simulation setup and procudure}

The numerical model formulation for representing the molecular structure of the chosen nanoparticles and the eutectic molecules was performed using Materials Studio (Accelrys, Inc_2008). Figure 3.3 shows a typical configuration used for the simulations. The simulation domain consists of a $(5,5)$ armchair SWCNT of diameter 
$6.78 \AA$ surrounding by liquid molecules. The liquid molecules considered in this simulation are molten salt eutectics. The simulation domain consisted of a rectangular box with square cross-section. For each simulation, the SWCNT is placed at the center of the simulation box. The liquid molecules are placed in the simulation domain to surround the SWCNT.

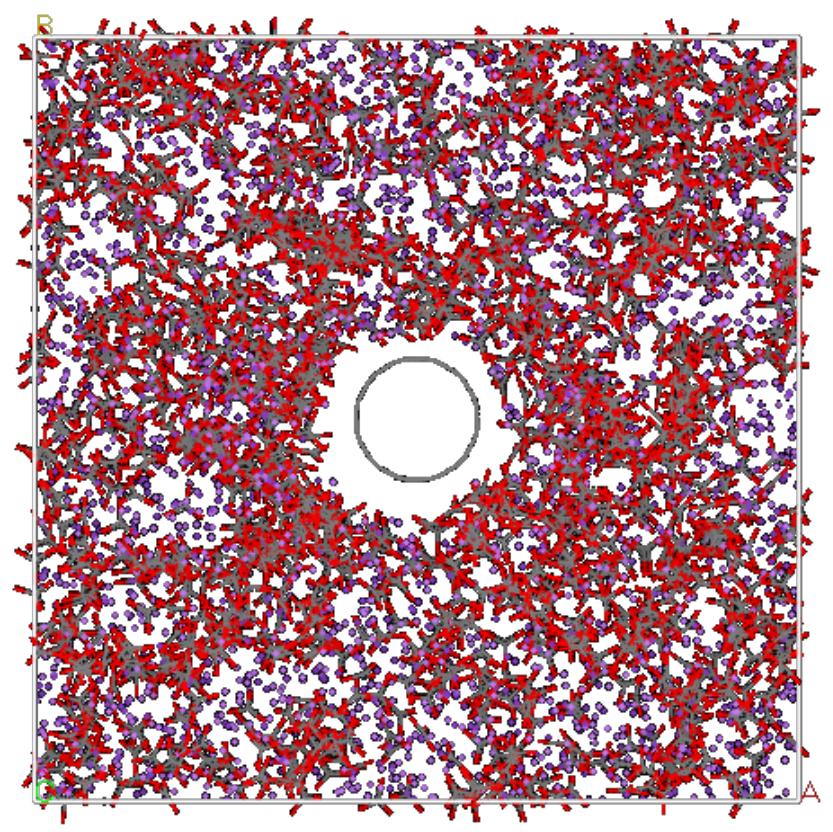

Figure 3.3. Simulation domain showing $(5,5)$ armchair SWCNT placed in the center and the solvent molecules of carbonate salt eutectic located around the crystal lattice of nanoparticle.

The Newton's equation of motion for each atom (i) of mass $(m)$ experiencing a force $(f)$, in an ensemble of atoms (total number of atoms in the ensemble is $N$ ) is expressed as in terms of the position $(r)$, acceleration and potential energy $(U)$ as: 


$$
f_{i}(t)=m \ddot{r}_{i}(t)=-\frac{\partial U\left(r^{N}\right)}{\partial r_{i}}
$$

Equation 2

Additional details on the system of equations is available in the book: "NanofinsScience and Applications" by N. Singh and D. Banerjee (Edition: 2014, Publisher: Springer, New York, NY). In this simulation, the force interaction between any two atoms is described by Lennard-Jones (L-J) potentials along with Coulombic force interaction for non-bonded atoms. ${ }^{29}$ The proposed model has been used in this study to account for the molecular interactions described by Equation 3, where $r$ is the distance between the center-of-masses of two atoms. The parameters used in the L-J model for the SWCNTs were obtained from previous studies reported in the literature, while the parameters for the carbonate eutectic molecules were obtained from the standard library (default values) that is included in Material Studio. ${ }^{64-66}$

$$
\begin{array}{ll}
E(r)=\frac{q_{i} \cdot q_{j}}{r}+4 \varepsilon\left[\left(\frac{\sigma}{r}\right)^{12}-\left(\frac{\sigma}{r}\right)^{6}\right] \quad \text { Equation } 3
\end{array}
$$

Since the molar ratio between lithium carbonate and potassium carbonate in the eutectic is a fixed value, the total number of molecules for each species was calculated in order to determine the size of the simulation box. Typically 1500 molecules of the eutectic were placed in the simulation domain. To account for the interaction between atoms of different species, the constants for the LJ potential were computed by the arithmetic mean, as shown in Equation 4 and Equation 5. 


$$
\begin{aligned}
\varepsilon_{i, j} & =\sqrt{\varepsilon_{i} \cdot \varepsilon_{j}} \\
\sigma_{i, j} & =\frac{\sigma_{i}+\sigma_{j}}{2}
\end{aligned}
$$

Equation 4

Equation 5

In addition, a cutoff radius of $12 \AA$ was used for calculating LJ interactions (in a previous study this value of cut-off radius was reported to be adequate for achieving the desired numerical accuracy for the predictions from the simulations ${ }^{29}$ ). For bonded interactions, bond stretching, bond-bending, and torsion were also incorporated in the numerical model, as shown in Equation 6. The model parameters for each atom (for both non-bonded and bonded interactions) are listed in Table 3.7.

$$
E=K_{s} \cdot\left(r-r_{0}\right)^{2}+K_{b} \cdot\left(\theta-\theta_{0}\right)+K_{t} \cdot(1+d \cdot \cos (n \phi)) \quad \text { Equation } 6
$$

\subsubsection{Numerical method}

Equation 1 is numerically integrated to obtain the velocity and position information. This enables the position, momentum and acceleration of each atom to be calculated as a function of time. In this study Verlet algorithm along with the Gear predictor algorithm is used to solve for the ensemble of equations enabling a direct solution of Equation 1. The position information at the next time step $r(t+\delta t)$ is obtained as a function of the position information for the current time step $r(t)$ and the previous time step, $r(t-\delta t)$ using Taylor series expansion as: 


$$
r(t+\delta t)=2 r(t)-r(t-\delta t)+\ddot{r}(t)(\delta t)^{2}+o\left[(\delta t)^{4}\right]
$$

Equation 7

In this equation the last term (acceleration) is calculated using Equation 1. The standard Verlet velocity algorithm is implemented in this study as:

$$
r(t+\delta t)=r(t)+\dot{r}(t)(\delta t)+\left(\frac{1}{2}\right) \ddot{r}(t)(\delta t)^{2}+o\left[(\delta t)^{3}\right]
$$

Equation 8

The velocity information in Equation 7 is used to calculate the kinetic energy of the ensemble of the equations which is then used to interpret the total energy, temperature and pressure of the system. Equation 7 enables the position, velocity and acceleration of the ensemble of atoms is obtained at the same time step. Since the order of global error is of the order of (dt) 2 it is imperative to choose a small enough time step to ensure that the error propagation with time step is minimized. However, too small time steps can be problematic due to potential domination of errors arising from propagation of truncation errors and round-off errors with time.

\subsubsection{Simulation procedure}

To initiate the simulation, the eutectic molecules are placed at random locations as well as in random orientations and the energy of the system is minimized using appropriate numerical procedure (described next). Since the molecules are placed randomly inside of the simulation domain, the atoms may be very close to each other or may even overlap, leading to non-physical configuration. Therefore, this initial state is not appropriate to 
run a simulation for calculating interactions between a particle and eutectic. Then, a minimization procedure is implemented initially to redistribute the atoms to causes an energy minimization step which causes the temperature of the system to be equal to $0 \mathrm{~K}$.

After the minimization process, a relaxation procedure is imposed on the system by using micro-canonical ensemble (or constant number, volume and energy ensemble, or "NVE" ensemble). Then, the system is brought to a fixed base temperature of $800 \mathrm{~K}$ in this simulation. This temperature is chosen since the melting point of the eutectic is $761 \mathrm{~K}$. Also, the system pressure is adjusted to atmospheric pressure by performing time integration as an isothermal-isobaric ensemble (or constant number, pressure, temperature, or "NPT" ensemble).

$$
\frac{T_{\text {reqd }}}{T_{\text {base }}}=\left(\frac{V_{\text {reqd }}}{V_{\text {base }}}\right)^{2}
$$

Equation 9

Following the equilibration process for reaching a uniform temperature of $800 \mathrm{~K}$, the temperature of the atoms of the SWCNT (which is placed at the center of the simulation domain) is instantaneously raised to $1400 \mathrm{~K}$ by directly scaling of the velocities of the atoms. The relationship between temperature and velocity is demonstrated in Equation 9, where, $T_{\text {reqd }}$ is the required temperature of the nanotube and $T_{\text {base }}$ is the base temperature, while $V_{\text {base }}$ is the initial velocity of the atoms in the nanotube, and $V_{\text {reqd }}$ is the scaled velocity. 
At this point, the ensemble is ready for starting the numerical simulation. In the simulations the values of the interfacial thermal resistance is estimated from the transient thermal response of the SWCNT. The system is equilibrated as an NVE ensemble by starting with the modified velocities of the SWCNT. As the system relaxes under constant energy, the energy from the SWCNT is slowly transferred to the eutectic molecules. As the system relaxes, after fixed time intervals, a snapshot of the numerical distribution of the velocities of the atoms is obtained, which is then used to calculate the temperature of the nanotube and the eutectic molecules. ${ }^{29,33}$ The temperature for the SWCNT and the eutectic molecules is calculated and then the temperature difference is plotted as a function of time, as shown in Figure 3.4. These Molecular Dynamics (MD) simulations have been performed by using Large-scale Atomic/ Molecular Massively Parallel Simulator (LAMMPS) distributed by Sandia National Laboratory (released on $4^{\text {th }}$ July, 2012).

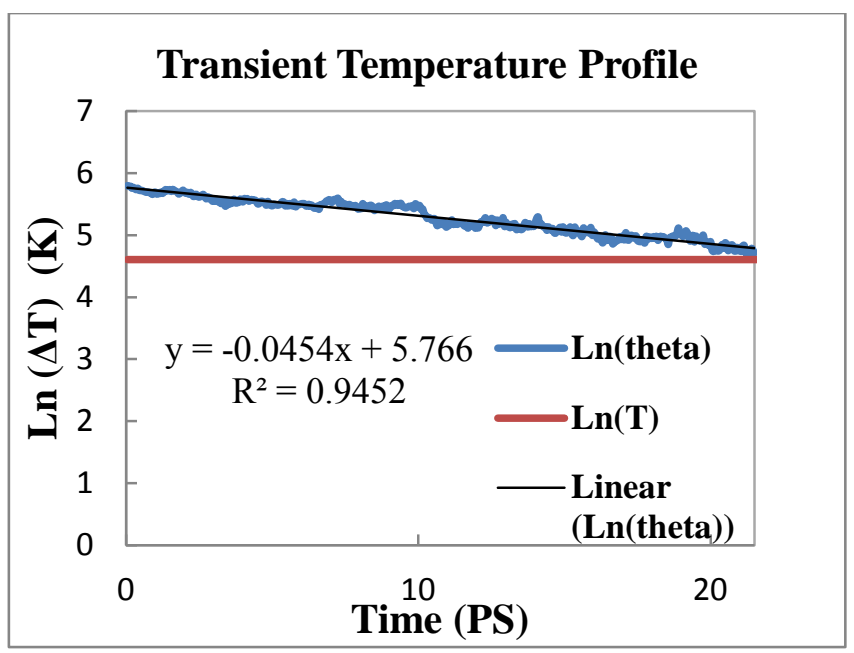

Figure 3.4. Transient temperature profile of a SWCNT obtained from the MD simulations. 


\section{SECTION 4. RESULTS AND DISCUSSION}

Lumped capacitance method has been used to calculate the value of interfacial thermal resistance (i.e., Kapitza Resistance, " $R$ "). The values of interfacial thermal resistance " $R$ " depend on the transient heat transfer between the SWCNT (at a higher temperature) and the molten salt eutectics (at a lower temperature, and is virtually unchanged due to larger values of the total thermal inertia of the ensemble of the fluid molecules). Using a similar numerical scheme, Huxtable et al. ${ }^{26}$ obtained the value of the interfacial thermal resistance between a single wall carbon nanotube and octane molecules. Singh et al. ${ }^{33}$ used the same method to calculate the interfacial thermal resistance between a single wall carbon nanotube and various matrixes, such as poly-alpha-olefin (PAO) oil that is typically used for avionics cooling and energy applications. However, the heat capacity used in these studies is fixed at a constant value of $5.6 \times 10^{-4} \mathrm{~J} / \mathrm{m}^{2} \cdot \mathrm{K}$; which is defined as the specific heat capacity per unit area of an atomic layer of SWCNT. In this study, the relationships between the specific heat capacity, surface area, and volume for each single wall carbon nanotube are not assumed to be constant. Hence, using lumped capacitance analysis, the interfacial thermal resistance " $R_{k}$ " between single wall carbon nanotube and eutectic molecules is described by Equation 10 below. 


$$
\tau=\frac{R_{k} \cdot C_{T}}{A_{c n t}}=\frac{R_{k} \cdot\left(\rho V c_{p}\right)}{A_{c n t}}
$$

Equation 10

Where, $C_{T}$ is the heat capacity of the nanotube and $A_{c n t}$ is the surface area of the carbon nanotube. As mentioned before, the ratio $C_{T} / A_{c n t}$ is approximately constant and is usually assumed to be fixed at a value of $5.6 \times 10^{-4} \mathrm{~J} / \mathrm{m}^{2} \cdot \mathrm{K}^{26,33}$ In order to calculate the interfacial thermal resistance " $R_{k}$ ", value of time constant $\tau$ is required. The required value of time constant was obtained by plotting the temperature decay of the SWCNT, as shown in Figure 4.3. Although all of three nano-particles in this study are SWCNT, there are small differences in the property values between the CNT lattices with the three different types of chirality. For instance, the density values for armchair, chiral, and zig-zag SWCNT, used in this study are $1.33 \mathrm{~g} / \mathrm{cm}^{3}, 1.40 \mathrm{~g} / \mathrm{cm}^{3}$, and 1.34 $\mathrm{g} / \mathrm{cm}^{3}$ respectively. ${ }^{67}$ In the course of the simulation steps, as the average temperature of the SWCNT decreases (especially below 900K), significant fluctuations in the temperature values were observed - compared to the linearized fit in the log-plot of the temperature transients (i.e., similar to plots in Figure 4.3). Hence, the time constant was obtained from the transient temperature plots by calculating the slope of the best-fit line for the temperature results obtained from the simulation. The temperature range employed for obtaining the value of interfacial thermal resistance " $R_{k}$ " in the numerical study was varied from the initial temperature of $1400 \mathrm{~K}$ (that was fixed for each simulation) to a final decay temperature of $900 \mathrm{~K}$ (even though the simulations could have continued to temperatures below $900 \mathrm{~K}$ ). From the numerical simulations, the 
values of the interfacial thermal resistance were estimated to be $7.90 \times 10^{-8} \mathrm{~m}^{2} \cdot \mathrm{K} / \mathrm{W}$ and $8.58 \times 10^{-8} \mathrm{~m}^{2} \cdot \mathrm{K} / \mathrm{W}$ for $(5,5)$ and $(6,6)$ armchair SWCNT. The values of the interfacial thermal resistance for zigzag and chiral SWCNTs are listed in following sections. The predicted values are found to be consistent with the values reported in the literature for both experimental measurements and numerical studies - which were reported to vary from $0.76 \times 10^{-8} \mathrm{~m}^{2} \cdot \mathrm{K} / \mathrm{W}$ to $20 \times 10^{-8} \mathrm{~m}^{2} \cdot \mathrm{K} / \mathrm{W}$. Table 4.2 shows the calculated interfacial thermal resistance of zig-zag SWCNTs. As shown in the table, the $(6,0)$ and $(9,0)$ zig-zag SWCNT has much higher value compared to the other zig-zag SWCNTs. The " $R_{k}$ " values were found to be sensitive to the structure of the CNT while the diameter of the CNT is found to weakly affect the values of " $R_{k}$ ". Higher values of interfacial thermal resistance were observed for the metallic single wall carbon nanotube. In contrast, the semi-conducting SWCNT were observed to have lower values of interfacial thermal resistance. An interesting trend is observed when comparison between armchair and zig-zag SWCNT is performed which has the same diameter and crystal structure. However, the interfacial thermal resistance is much higher in zig-zag SWCNT. For example, the interfacial thermal resistance of $(9,0)$ metallic zig-zag SWCNT with a diameter of $7.05 \AA$ is $11.47 \times 10^{-8} \mathrm{~m}^{2} \cdot \mathrm{K} / \mathrm{W}$. In contrast the $(5,5)$ metallic armchair SWCNT with the diameter of $6.78 \AA$ has a value of $7.90 \times 10^{-8} \mathrm{~m}^{2} \cdot \mathrm{K} / \mathrm{W}$. From the calculated values of the interfacial thermal resistance of chiral SWCNT shown in following sections, same trend can be observed, for the metallic SWCNT, which have higher values of " $R_{k}$ " compared to that of the semi-conductor SWCNTs. However, no significant differences in the values of " $R_{k}$ " for armchair SWCNT were observed since 
they have metallic property. The average values of " $R_{k}$ " for metallic CNT is observed to be $\sim 45 \%-50 \%$ higher than for the semi-conducting CNT. This is potentially due to higher vibrational frequencies of the metallic CNT compared to that of the semi-conducting CNT - resulting in higher impedance mismatch with the solvent molecules.

\subsection{Interfacial thermal resistance and critical particle size of armchair SWCNT}

As mentioned in the Introduction section, in spite of extensive studies in the literature for estimating the values of interfacial thermal resistance " $R_{k}$ " for mixtures of CNT with various solvents, the prior studies have been restricted essentially to armchair CNT with the metallic properties, and primarily to $(5,5)$ CNT crystal. Hence, this study is focused on the MD simulation of chirality values other than armchair carbon nanotube. In previous studies in the literature, the critical diameter of nanoparticles for enhancing thermal conductivity was expressed by the Equation 1 (in Chapter 1), where $k_{f}$ represent to the thermal conductivity of the solvent. In this study, only $(5,5)$ and $(6,6)$ armchair carbon nanotube configurations were analyzed in order to validate the numerical accuracy of the method/model used in this simulation (and to compare with the previous results reported in the literature). The calculated values of the interfacial thermal resistance are listed in Table 4.1.

Figure 4.1 shows the simulation domain after the MD analysis. The figure shows that the $(5,5)$ armchair carbon nanotube is still located in the center of the simulation box, and 
the adsorbed layer of fluid molecules (compressed phase) exists at the interface between the carbon nanotube and the solvent molecules. Similar trends were obtained in $(6,6)$ armchair carbon nanotube as well.

Table 4.1. Interfacial thermal resistance and critical size of Armchair SWCNTs

\begin{tabular}{|c|c|c|c|c|c|}
\hline SWCNT & Diameter & Length & Property & $\begin{array}{c}\mathrm{R}_{\mathrm{k}} \\
{\left[\mathrm{m}^{2} \cdot \mathrm{K} / \mathrm{W}\right]}\end{array}$ & $\begin{array}{c}\mathrm{d}_{\mathrm{c}} \\
{[\mathrm{nm}]}\end{array}$ \\
\cline { 1 - 2 }$(5,5)$ & $6.78 \AA$ & $49.19 \AA$ & Metallic & $7.90 \mathrm{e}-08$ & 118.5 \\
\cline { 1 - 1 } \cline { 5 - 6 }$(6,6)$ & $8.14 \AA$ & & Metallic & $8.58 \mathrm{e}-08$ & 128.7 \\
\hline
\end{tabular}

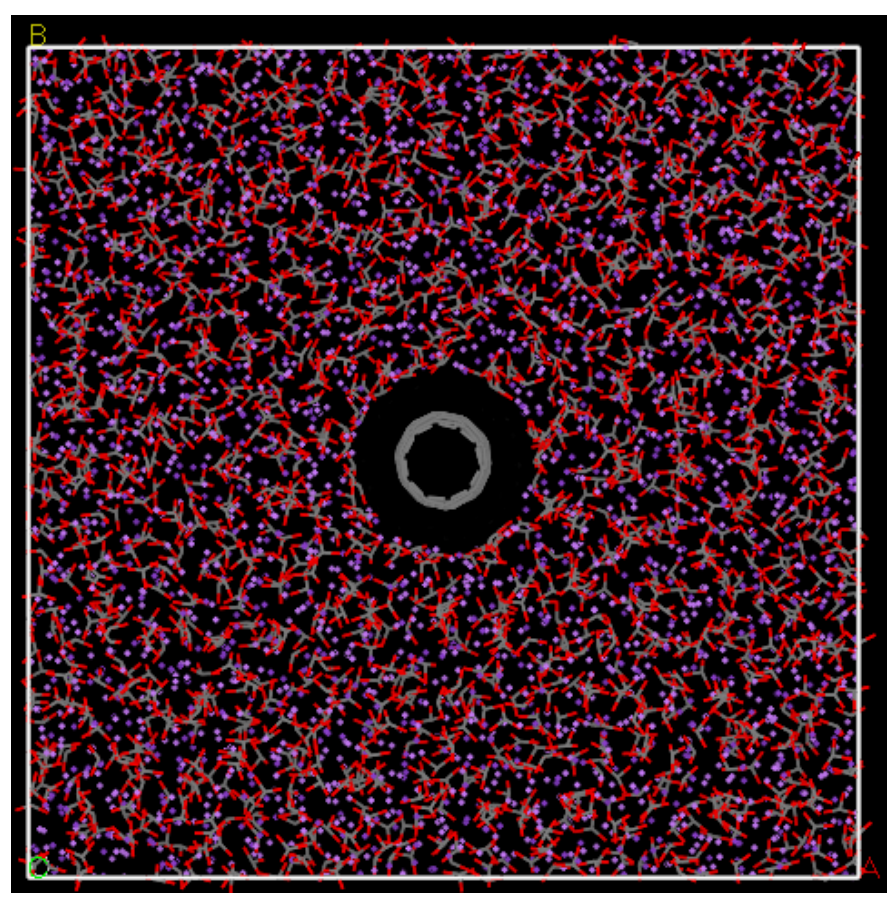

Figure 4.1 Simulation domain showing $(5,5)$ armchair SWCNT placed in the center and the solvent molecules of carbonate salt eutectic located around the crystal lattice of the nanotube. 
Figure 4.2 shows the spatial density distribution of the $(5,5)$ armchair carbon nanotube, where the abscissa represents the radial location starting from the center of the carbon nanotube. Considering that the radius of the $(5,5)$ armchair carbon nanotube is $3.39 \AA$, there will be no fluid molecules for a spherical region of $\sim 7.4 \AA$ diameter within the simulation domain, due to the presence of the carbon nanotube which will not allow the solvent molecules go inside the tube and the thickness of the compressed layer is almost the same order of magnitude as the radius of the carbon nanotube. Hence, the density plot shows values only after a center distance, $\sim 6 \AA$ for $(5,5)$ armchair carbon nanotube. Similar trends were observed for $(6,6)$ armchair carbon nanotube.

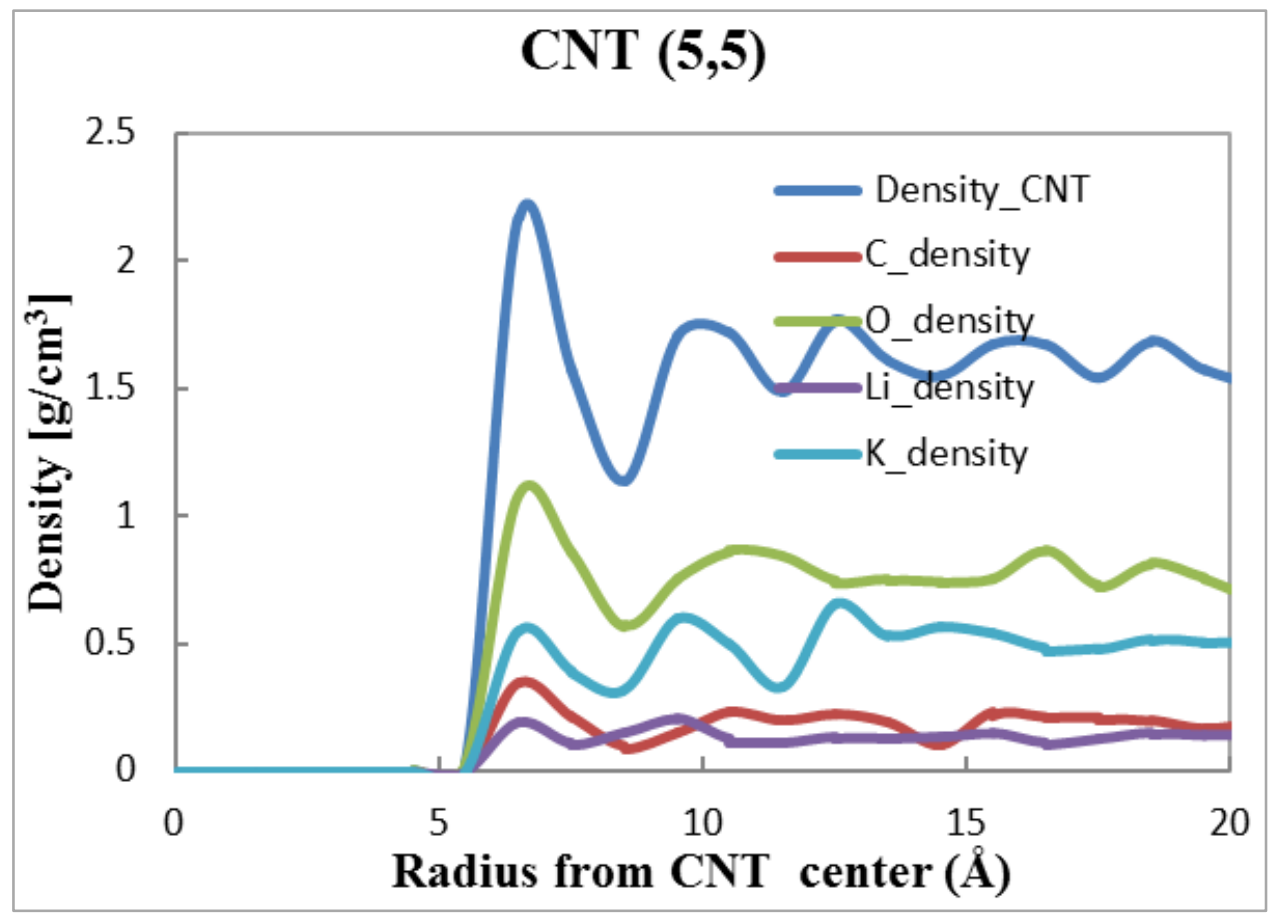

Figure 4.2 Spatial density distribution around a $(5,5)$ armchair SWCNT that is surrounded by molten salts molecules. 
Figure 4.3 shows the temperature decay plot, where the slope of the best-fit line in the plot is used to calculate the interfacial thermal resistance. The governing equations and calculation method were introduced in previous sections. Similar trends were observed for $(6,6)$ armchair carbon nanotube.

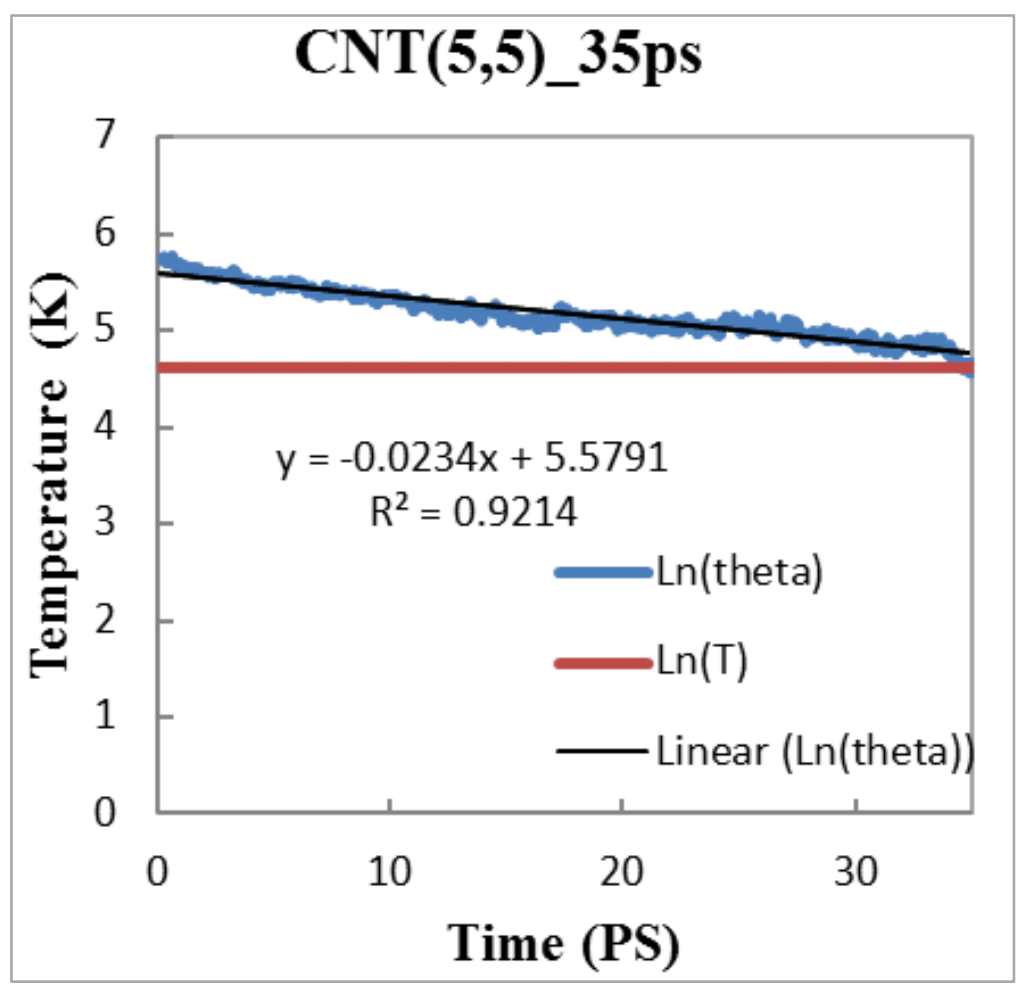

Figure 4.3 Temperature decay plot of a $(5,5)$ armchair SWCNT that is surrounded by molten salts molecules.

A plot of interfacial thermal resistance of armchair SWCNTs is shown in Figure 4.4. The interfacial thermal resistance will increase as the diameter/size of the armchair carbon nanotube increases. 


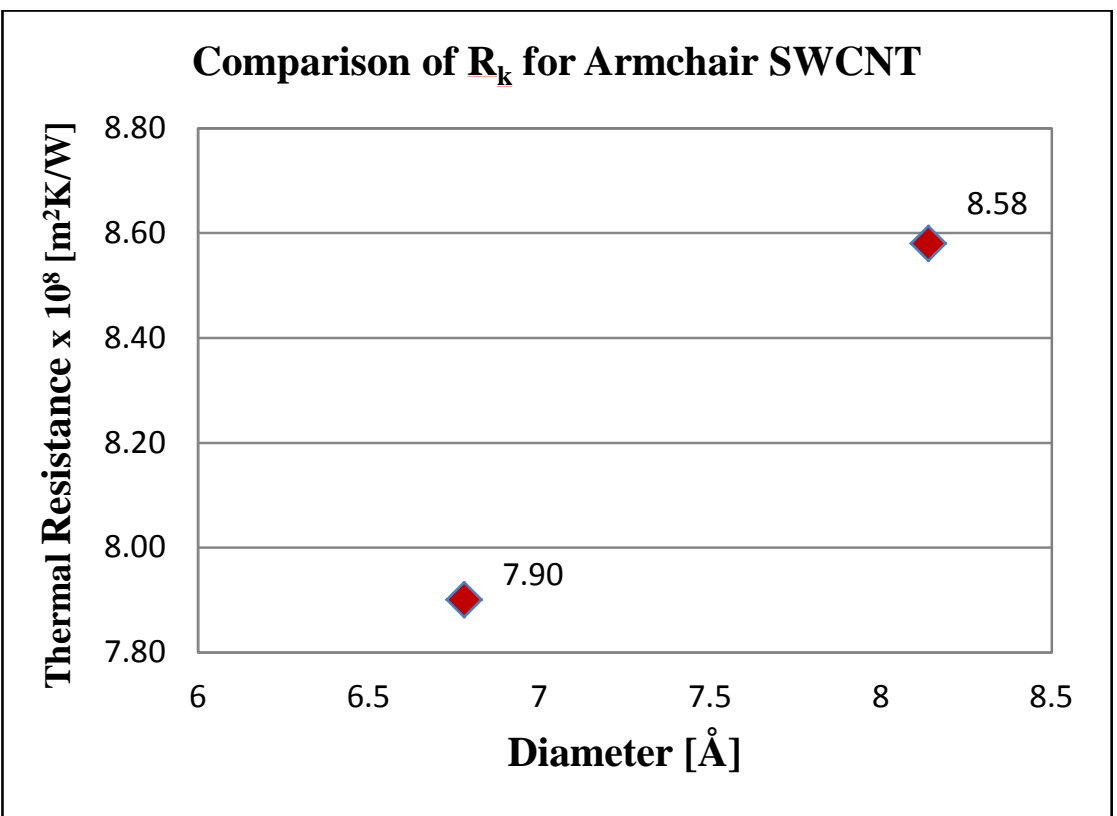

Figure 4.4 Plot of interfacial thermal resistance of Armchair SWCNTs.

\subsection{Interfacial thermal resistance and critical particle size of zig-zag SWCNT}

As mentioned previously, the zig-zag carbon nanotube is defined as $\mathrm{m}=0$, which corresponds to the top horizontal dotted line in Figure 1.9. Considering that there are two semi-conductive zig-zag carbon nanotubes for every metallic zig-zag carbon nanotube. The calculated values of the interfacial thermal resistance were listed in the Table 4.2.

Considering that the temperature decay plots for the different chirality values are all similar and display similar trends, so only a select few plots for the zig-zag carbon nanotube have been shown below. Figure 4.5 shows the simulation domain after the completion of the MD simulation. 
Table 4.2. Interfacial thermal resistance and critical size of Zig-zag SWCNTs

\begin{tabular}{|c|c|c|c|c|c|}
\hline SWCNT & Diameter & Length & Property & $\begin{array}{c}\mathrm{R}_{\mathrm{k}} \\
{\left[\mathrm{m}^{2} \cdot \mathrm{K} / \mathrm{W}\right]}\end{array}$ & $\begin{array}{c}\mathrm{d}_{\mathrm{c}} \\
{[\mathrm{nm}]}\end{array}$ \\
\hline$(5,0)$ & $3.91 \AA$ & \multirow{6}{*}{$\begin{array}{l}42.60 \AA \\
\text { (10 unit) }\end{array}$} & Semi-conductor & $5.35 \mathrm{e}-08$ & 80.25 \\
\hline$(6,0)$ & $4.70 \AA$ & & Metallic & $10.80 \mathrm{e}-08$ & 162.00 \\
\hline$(7,0)$ & $5.48 \AA$ & & Semi-conductor & $6.75 \mathrm{e}-08$ & 101.25 \\
\hline$(8,0)$ & $6.26 \AA$ & & Semi-conductor & $6.63 \mathrm{e}-08$ & 99.45 \\
\hline$(9,0)$ & $7.05 \AA$ & & Metallic & $11.47 \mathrm{e}-08$ & 172.05 \\
\hline$(10,0)$ & $7.83 \AA$ & & Semi-conductor & $6.77 \mathrm{e}-08$ & 101.05 \\
\hline
\end{tabular}

As shown in the figure, the $(10,0)$ armchair carbon nanotube is located in the center of the simulation box, and the compressed layer forms at the interface between the carbon nanotube and the solvent molecules.

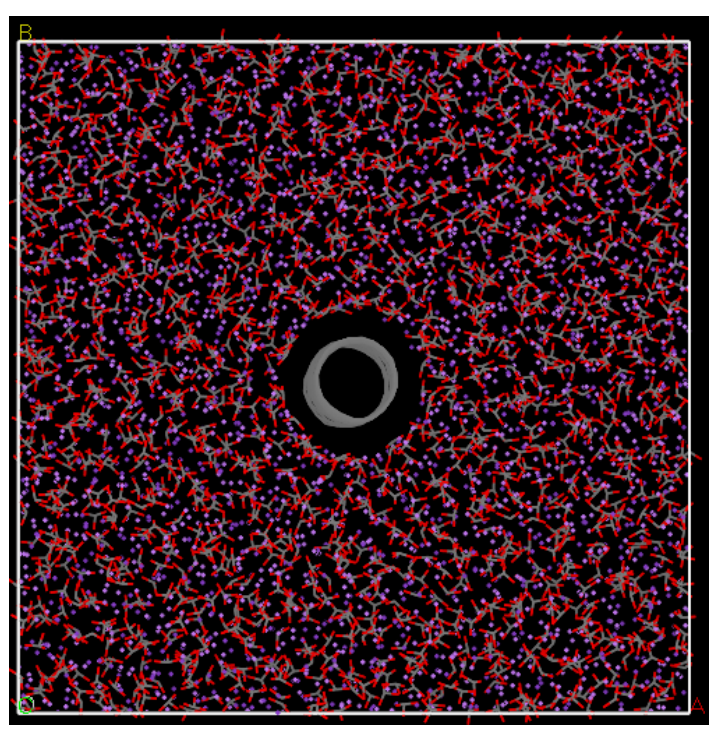

Figure 4.5 Simulation domain showing a $(10,0)$ zig-zag SWCNT placed in the center and the solvent molecules of carbonate salt eutectic located around the crystal lattice of the nanotube. 
Figure 4.6 shows the spatial density distribution of the $(10,0)$ zig-zag carbon nanotube, the abscissa represents the radial location starting from the center of the carbon nanotube. Considering that the radius of the $(10,0)$ zig-zag carbon nanotube is $3.925 \AA$, no molecules will be located within a spherical region of diameter $\sim 7.85 \AA$.

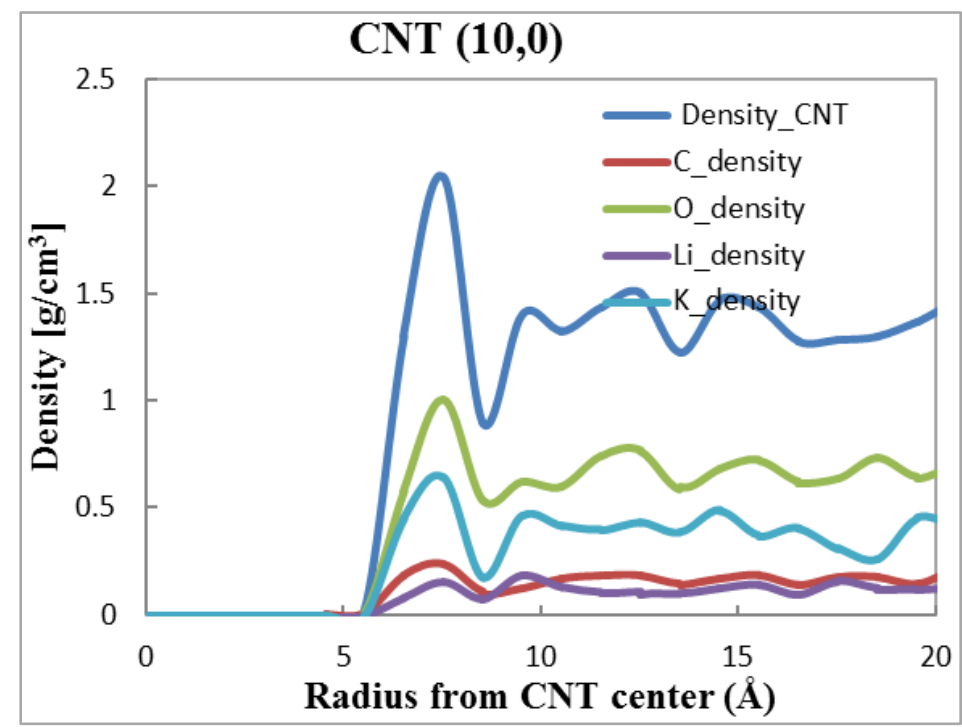

Figure 4.6 Spatial density distribution around a $(10,0)$ zig-zag SWCNT that is surrounded by molten salts molecules.

Figure 4.7 shows the temperature decay plot, where the slope of the best-fit line is utilized to obtain the value for the interfacial thermal resistance.

A plot of interfacial thermal resistance of $(10,0)$ zig-zag SWCNT is shown in Figure 4.8. The plot shows that the value of interfacial thermal resistance increases with the diameter/size of the zig-zag carbon nanotube. 


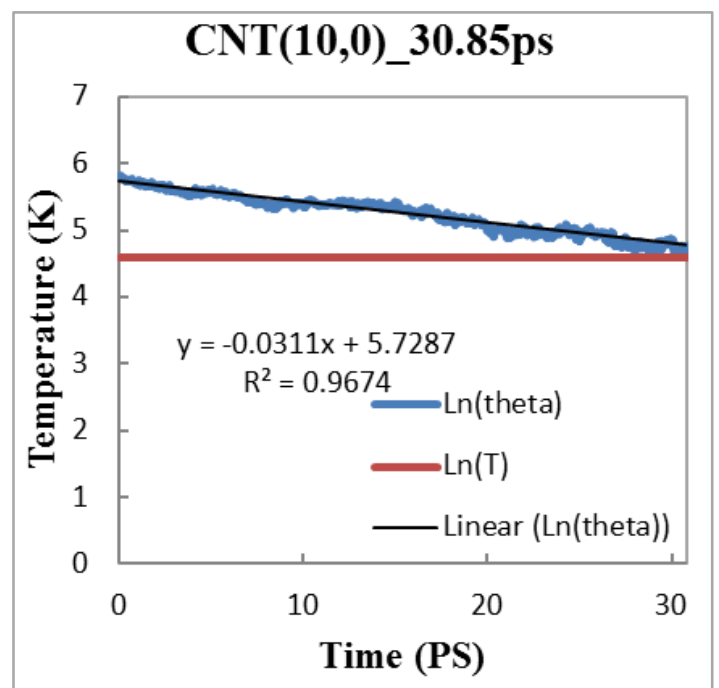

Figure 4.7 Temperature decay plot for a $(10,0)$ zig-zag SWCNT that is surrounded by molten salts molecules.

However, considering that carbon nanotube in zig-zag category can be either semi-conducting or metallic, it is observed that the metallic carbon nanotubes have much higher value of the interfacial thermal resistance compared to that of the semi-conducting carbon nanotubes.

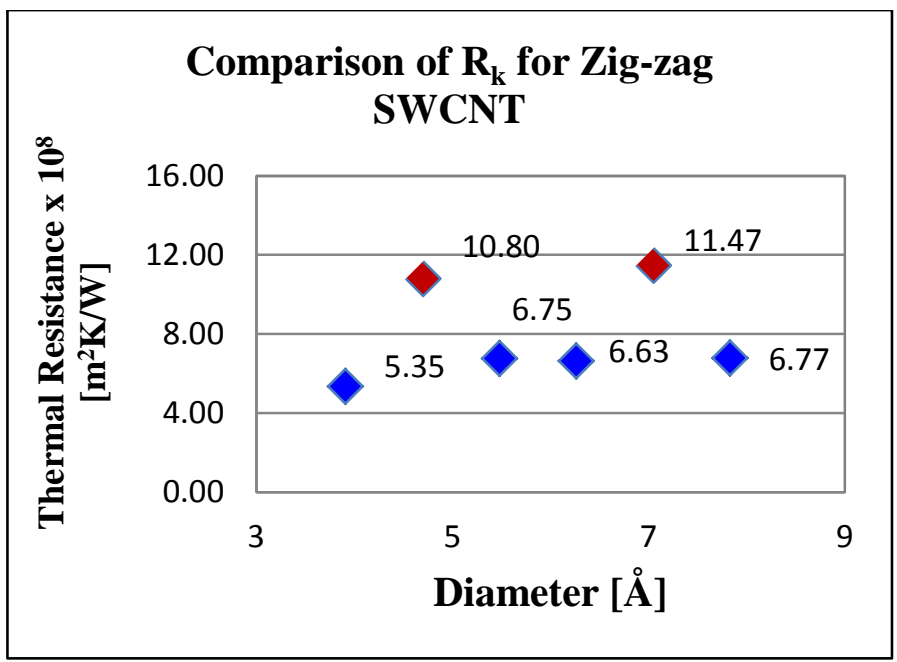

Figure 4.8 Plot of interfacial thermal resistance of Zig-zag SWCNTs. 
For enabling a consistent evaluation the Kapitza resistance values for metallic zig-zag SWCNT is compared with that of armchair SWCNT. The metallic zig-zag SWCNT have higher values of Kapitza resistance compared to that of the armchair SWCNT, plot of comparison shown in Figure 4.9. Also for similar size ranges of the metallic zig-zag and armchair CNT, the interfacial thermal resistance of metallic zig-zag SWCNT is almost $45 \%$ higher than that of the armchair SWCNT. This is a significant observation obtained in this study, since higher interfacial thermal resistance may be a potential option to modulate the heat transfer in various applications.

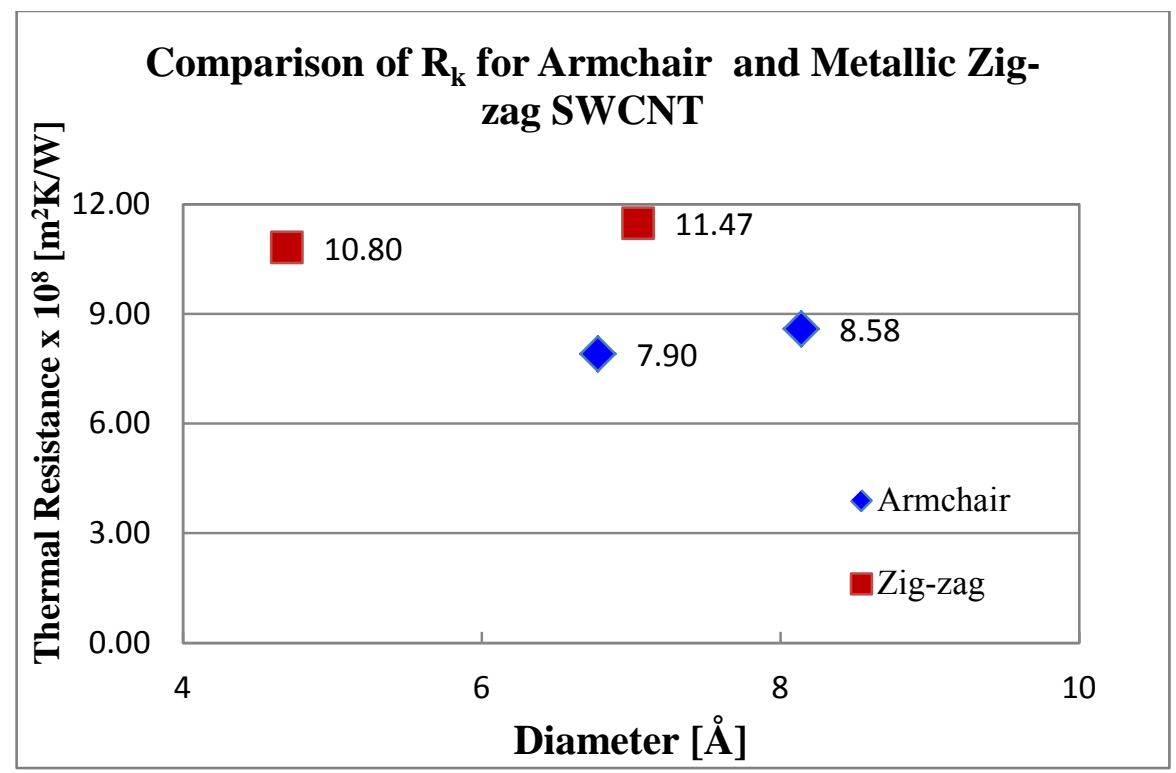

Figure 4.9 Comparison of interfacial thermal resistance between armchair and Zig-zag (metallic) SWCNTs. 


\subsection{Interfacial thermal resistance and critical particle size of chiral SWCNT}

As discussed in previous section, the chirality values that do not belong to the top horizontal dotted line and the $30^{\circ}$ inclined dotted line is classified to be chiral carbon nanotubes. The structural features of chiral carbon nanotube are similar to that of the zig-zag carbon nanotube. Only 1 in 3 chiral carbon nanotubes are metallic, while the other 2 in 3 chiral nanotubes are semiconducting. Hence the next set of investigations was performed based on this classification. The details of the results obtained from these investigations are described below.

\subsection{1. (n, 1) Chiral SWCNT}

From Figure 1.9 , it is observed that the $(n, 1)$ chiral carbon nanotubes are located on the second horizontal dotted line, which is right below the zig-zag dotted line. The calculated values of the interfacial thermal resistance are listed in Table 4.3.

Considering that the thermal decay plots are all similar and have similar trends, only a few select plots for the $(n, 1)$ chiral carbon nanotube have been shown below. Figure 4.10 shows the simulation domain after performing the MD simulations. The figure shows that the $(10,1)$ chiral carbon nanotube is located in the center of the simulation box, and compressed phase forms at the interface between the carbon nanotube and the solvent molecules. Similar trends were observed for the simulation results from other $(\mathrm{n}, 1)$ chiral carbon nanotubes as well. 
Table 4.3. Interfacial thermal resistance and critical particle of Chiral $(n, 1)$ SWCNT

\begin{tabular}{|c|c|c|l|c|c|}
\hline SWCNT & Diameter & Length & Property & $\begin{array}{c}\mathrm{R}_{\mathrm{k}} \\
{\left[\mathrm{m}^{2} \cdot \mathrm{K} / \mathrm{W}\right]}\end{array}$ & $\begin{array}{c}\mathrm{d}_{\mathrm{c}} \\
{[\mathrm{nm}]}\end{array}$ \\
\hline$(5,1)$ & $4.36 \AA$ & $\begin{array}{c}47.44 \AA \\
(2 \mathrm{unit})\end{array}$ & Semi-conductor & $4.83 \mathrm{e}-08$ & 72.45 \\
\hline$(6,1)$ & $5.13 \AA$ & $\begin{array}{c}55.87 \AA \\
(2 \mathrm{unit})\end{array}$ & Semi-conductor & $5.39 \mathrm{e}-08$ & 80.85 \\
\hline$(9,1)$ & $7.47 \AA$ & $\begin{array}{c}81.28 \AA \\
(2 \mathrm{unit})\end{array}$ & Semi-conductor & $5.76 \mathrm{e}-8$ & 86.40 \\
\hline$(10,1)$ & $8.25 \AA$ & $\begin{array}{c}89.76 \AA \\
(2 \mathrm{unit})\end{array}$ & Metallic & $7.36 \mathrm{e}-8$ & 110.4 \\
\hline
\end{tabular}

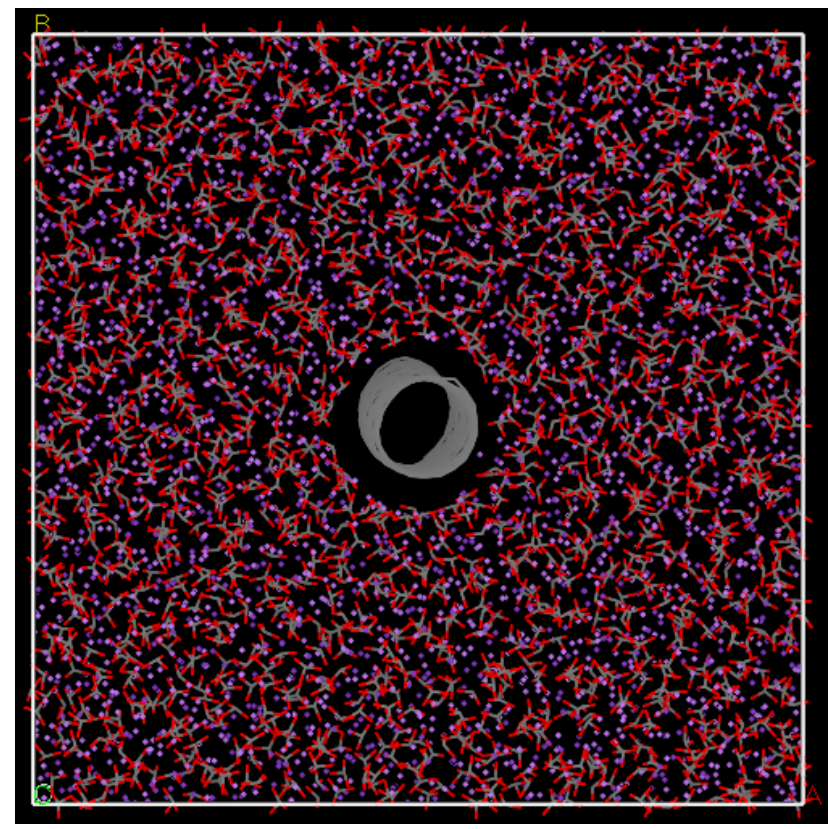

Figure 4.10 Simulation domain showing a $(10,1)$ chiral SWCNT placed in the center and the solvent molecules of carbonate salt eutectic located around the crystal lattice of the nanotube. 
Figure 4.11 shows the spatial density distribution of the $(10,1)$ chiral carbon nanotube, where the abscissa represents the radial location starting from the center of the carbon nanotube. Considering that the radius of the $(10,1)$ chiral carbon nanotube is $4.125 \AA$, no molecules exist in a spherical region with a diameter of $\sim 8.25 \AA$. Similar trends were observed for simulation results obtained with other $(\mathrm{n}, 1)$ chiral carbon nanotubes as well. Figure 4.12 shows the temperature decay plot.

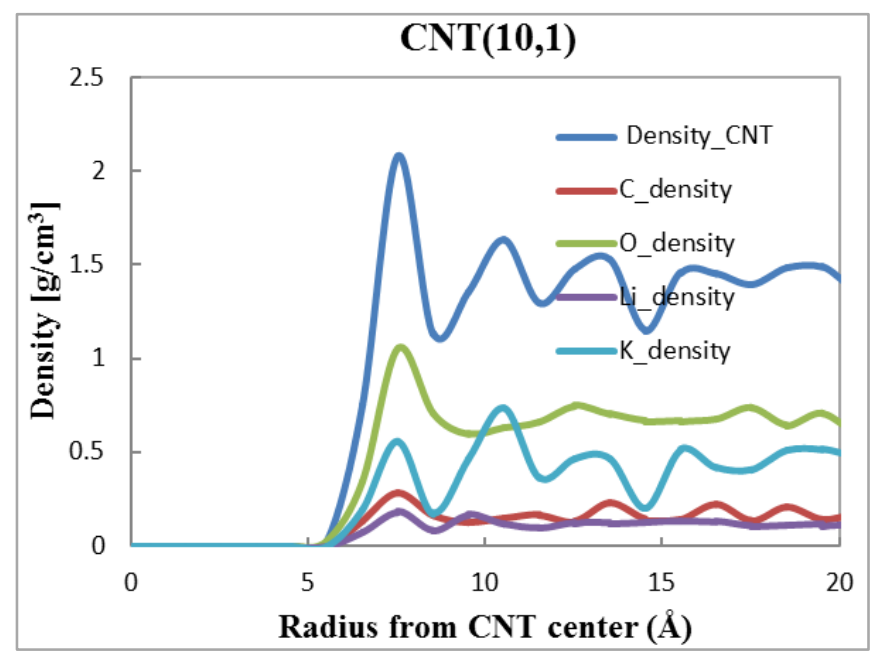

Figure 4.11 Spatial density distribution around a $(10,1)$ chiral SWCNT that is surrounded by molten salts molecules.

A plot of interfacial thermal resistance of $(n, 1)$ chiral SWCNT is shown in Figure 4.13. The plot shows that the interfacial thermal resistance increases as the diameter/size of the $(n, 1)$ chiral carbon nanotube increases. However, considering that there are both metallic and semiconducting carbon nanotubes for $(\mathrm{n}, 1)$ chiral category, it is observed that the metallic carbon nanotubes have significantly higher values of the interfacial thermal resistance compared to that of the semiconducting carbon nanotubes. 


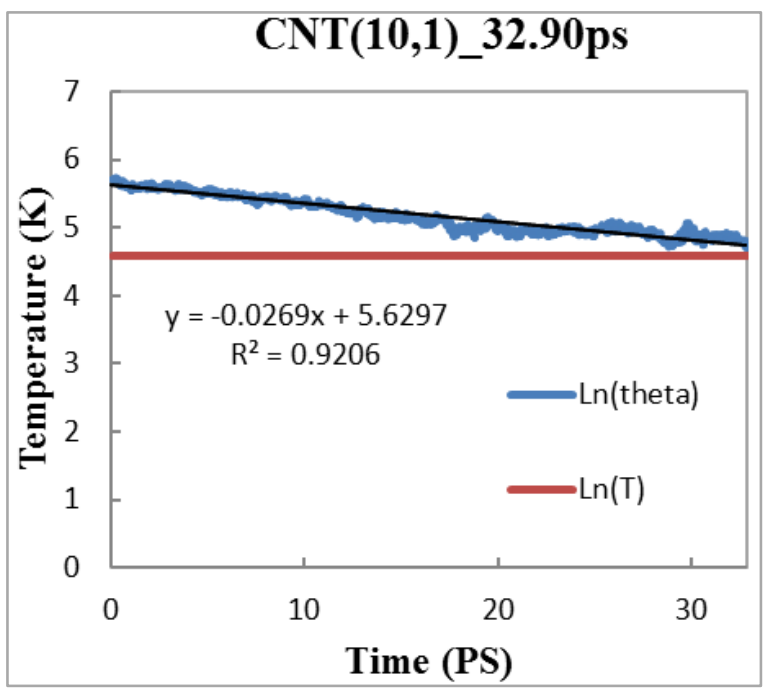

Figure 4.12 Temperature decay plot for a $(10,1)$ chiral SWCNT that is surrounded by molten salts molecules.

However, unlike the simulations involving the armchair and zig-zag carbon nanotubes, the chiral nanotubes are observed to be more susceptible to unstable situations, such as the disconnected sections of carbon nanotube occurring during the simulations. This issue will discuss later in this thesis.

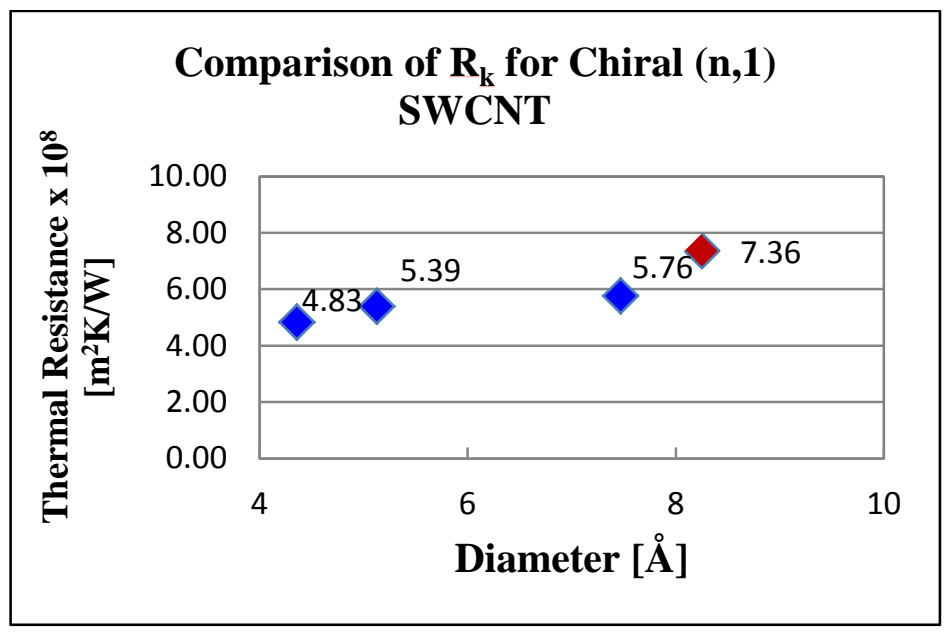

Figure 4.13 Plot of interfacial thermal resistance of $(n, 1)$ Chiral SWCNTs. 


\subsection{2. (n, 2) Chiral SWCNT}

From Figure 1.9 it is observed that the $(\mathrm{n}, 2)$ chiral carbon nanotubes are located on the third horizontal dotted line, which is located right below the $(n, 1)$ dotted line. The calculated values of the interfacial thermal resistance are listed in the Table 4.4.

Table 4.4. Interfacial thermal resistance and critical particle size of Chiral (n, 2) SWCNT

\begin{tabular}{|c|c|c|l|c|c|}
\hline SWCNT & Diameter & Length & Property & $\begin{array}{c}\mathrm{R}_{\mathrm{k}} \\
{\left[\mathrm{m}^{2} \cdot \mathrm{K} / \mathrm{W}\right]}\end{array}$ & $\begin{array}{c}\mathrm{d}_{\mathrm{c}} \\
{[\mathrm{nm}]}\end{array}$ \\
\hline$(5,2)$ & $4.89 \AA$ & $\begin{array}{c}53.51 \AA \\
(2 \mathrm{unit})\end{array}$ & Metallic & $7.27 \mathrm{e}-08$ & 109.05 \\
\hline$(6,2)$ & $5.65 \AA$ & $\begin{array}{c}30.72 \AA \\
(2 \mathrm{unit})\end{array}$ & Semi-conductor & $5.38 \mathrm{e}-08$ & 80.70 \\
\hline$(7,2)$ & $6.41 \AA$ & $\begin{array}{c}69.74 \AA \\
(2 \mathrm{unit})\end{array}$ & Semi-conductor & $6.15 \mathrm{e}-08$ & 92.25 \\
\hline$(8,2)$ & $7.18 \AA$ & $\begin{array}{c}39.04 \AA \\
(2 \text { unit })\end{array}$ & Metallic & $8.24 \mathrm{e}-8$ & 123.60 \\
\hline$(9,2)$ & $7.95 \AA$ & $\begin{array}{c}43.23 \AA \\
(1 \mathrm{unit})\end{array}$ & Semi-conductor & $4.81 \mathrm{e}-8$ & 72.15 \\
\hline$(10,2)$ & $8.72 \AA$ & $\begin{array}{c}47.44 \AA \\
(2 \text { unit })\end{array}$ & Semi-conductor & $4.85 \mathrm{e}-8$ & 72.15 \\
\hline
\end{tabular}

Figure 4.14 shows the simulation domain obtained upon the completion of the MD simulations. The figure shows that the $(8,2)$ chiral carbon nanotube is located in the center of the simulation box and compressed phase forms at the interface between the carbon nanotube and the solvent molecules. Similar trends were observed for the simulation results from other $(\mathrm{n}, 2)$ chiral carbon nanotube as well. 


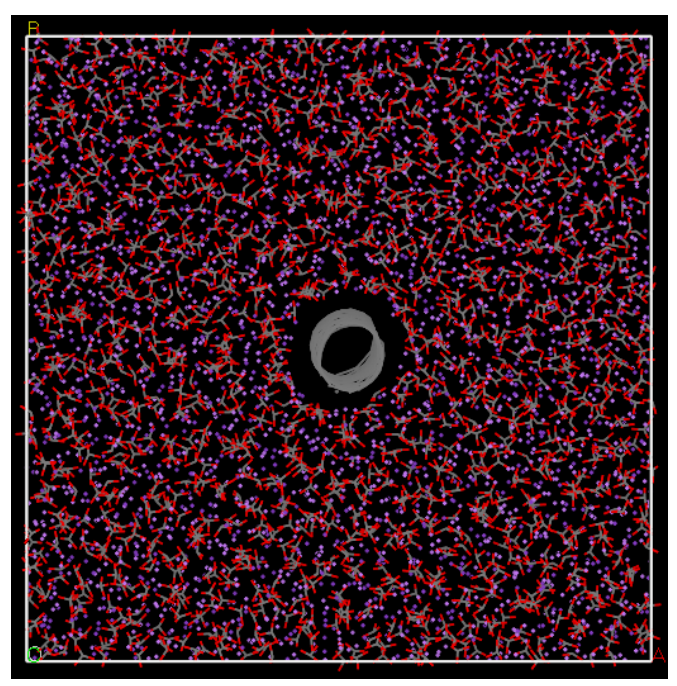

Figure 4.14 Simulation domain showing a $(8,2)$ chiral SWCNT placed in the center and the solvent molecules of carbonate salt eutectic located around the crystal lattice of a nanotube.

Figure 4.15 shows the spatial density distribution of the $(8,2)$ chiral carbon nanotube, where the abscissa represents the radial location starting from the center of the carbon nanotube.

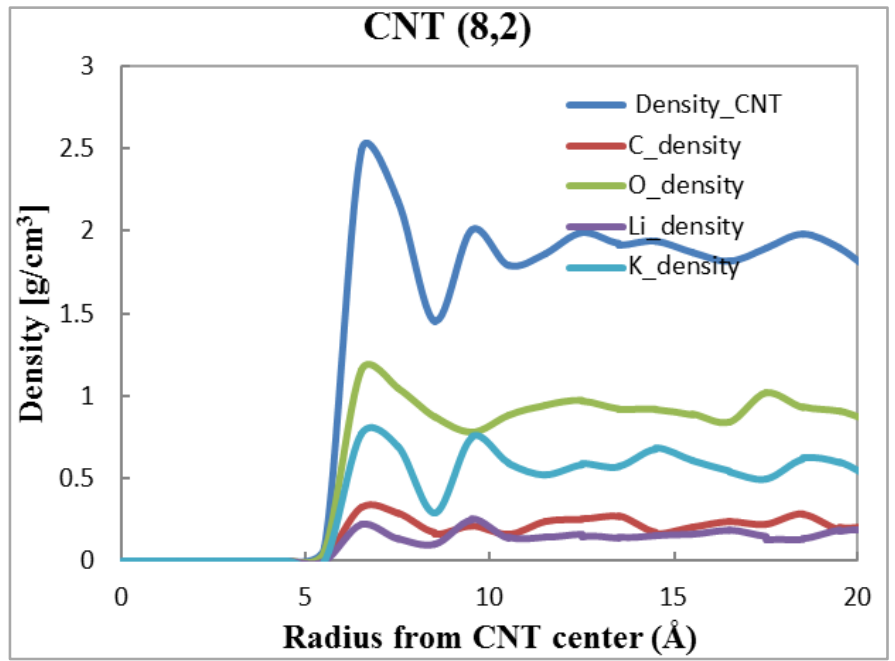

Figure 4.15 Spatial density distribution around a $(8,2)$ chiral SWCNT that is surrounded by molten salts molecules. 
Considering that the radius of the $(8,2)$ chiral carbon nanotube is $3.59 \AA$, no molecules exist in a spherical volume with a diameter of $\sim 7.2 \AA$. Figure 4.16 shows the temperature decay plot. Similar trends were observed for simulation results obtained with other (n, 2) chiral carbon nanotubes as well.

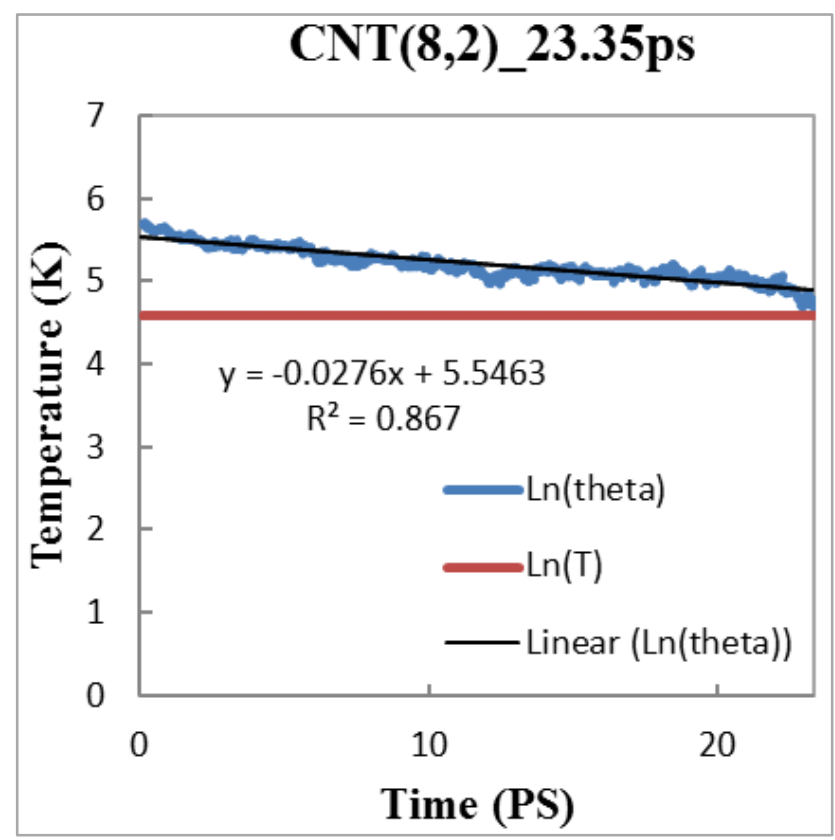

Figure 4.16 Temperature decay plot for a $(8,2)$ chiral SWCNT that is surrounded by molten salts molecules.

A plot of the interfacial thermal resistance of $(n, 2)$ chiral SWCNT is shown in Figure 4.17. The plot shows that the interfacial thermal resistance increases as the diameter/size of the $(n, 2)$ chiral carbon nanotube increases. However, considering that there are both metallic and semiconducting carbon nanotubes for the $(n, 2)$ chiral category, it is observed that the metallic carbon nanotubes have significantly higher values of the interfacial thermal resistance compared to that of the semiconducting 
carbon nanotubes. However, unlike the simulations involving the armchair and zig-zag carbon nanotubes, the chiral nanotubes are observed to be more susceptible to unstable situations, such as, the disconnected sections of the carbon nanotube occurring during the simulations. This issue will be discussed later in the thesis.

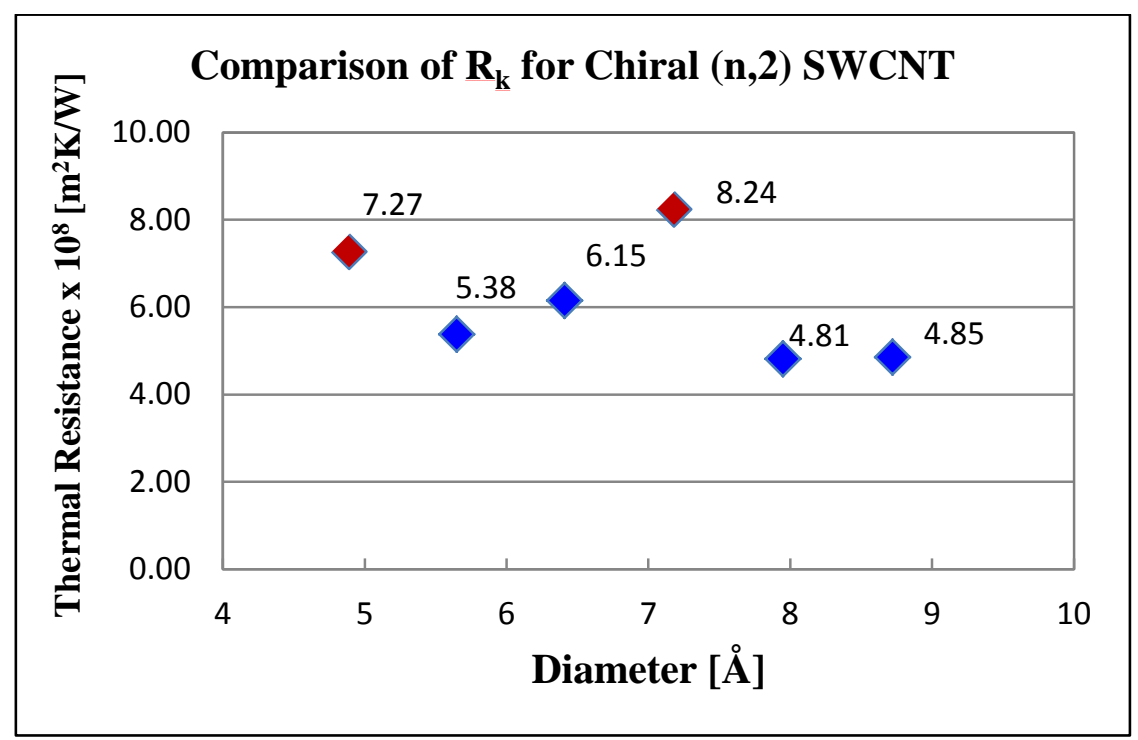

Figure 4.17 Plot of interfacial thermal resistance of (n, 2) Chiral SWCNTs.

\subsection{3. (n, 3) Chiral SWCNT}

From Figure 1.9 it is observed that the $(n, 3)$ chiral carbon nanotubes are located on the fourth horizontal dotted line, which is right below the $(\mathrm{n}, 2)$ dotted line. The calculated values of the interfacial thermal resistance are listed in the Table 4.5.

Figure 4.18 shows the simulation domain obtained upon the completion of the MD simulations. The figure shows that the $(8,3)$ chiral carbon nanotube is located in the 
center of the simulation box and compressed phase forms at the interface between the carbon nanotube and the solvent molecules.

Table 4.5. Interfacial thermal resistance of Chiral (n, 3) SWCNT

\begin{tabular}{|c|c|c|c|c|c|}
\hline SWCNT & Diameter & Length & Property & $\begin{array}{c}\mathrm{R}_{\mathrm{k}} \\
{\left[\mathrm{m}^{2} \cdot \mathrm{K} / \mathrm{W}\right]}\end{array}$ & $\begin{array}{c}\mathrm{d}_{\mathrm{c}} \\
{[\mathrm{nm}]}\end{array}$ \\
\hline$(7,3)$ & $6.96 \AA$ & $\begin{array}{c}75.73 \AA \\
(2 \text { unit })\end{array}$ & Semi-conductor & $5.92 \mathrm{e}-8$ & 88.80 \\
\hline$(8,3)$ & $7.71 \AA$ & $\begin{array}{c}41.96 \AA \\
(1 \text { unit })\end{array}$ & Semi-conductor & $4.39 \mathrm{e}-8$ & 65.85 \\
\hline$(9,3)$ & $8.47 \AA$ & $\begin{array}{c}46.08 \AA \\
(3 \text { unit })\end{array}$ & Metallic & $7.22 \mathrm{e}-8$ & 108.3 \\
\hline
\end{tabular}

Similar trends were observed for the simulation results from other $(\mathrm{n}, 3)$ chiral carbon nanotubes as well.

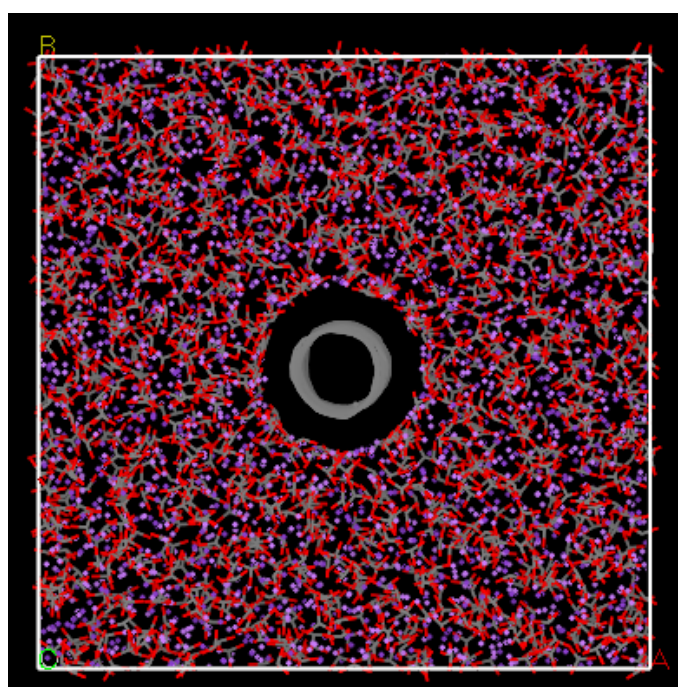

Figure 4.18 Simulation domain showing $(7,3)$ chiral SWCNT placed in the center and the solvent molecules of carbonate salt eutectic located around the crystal of a nanotube. 
Figure 4.19 shows the spatial density distribution of the $(7,3)$ chiral carbon nanotube, where the abscissa represents the radial location starting from the center of the carbon nanotube. Considering that the radius of the $(8,2)$ chiral carbon nanotube is $3.48 \AA$, no molecules exist in a spherical volume with a diameter of $\sim 7 \AA$. Figure 4.20 shows the temperature decay plot. Similar trends were observed for simulation results obtained with other $(\mathrm{n}, 3)$ chiral carbon nanotubes as well.

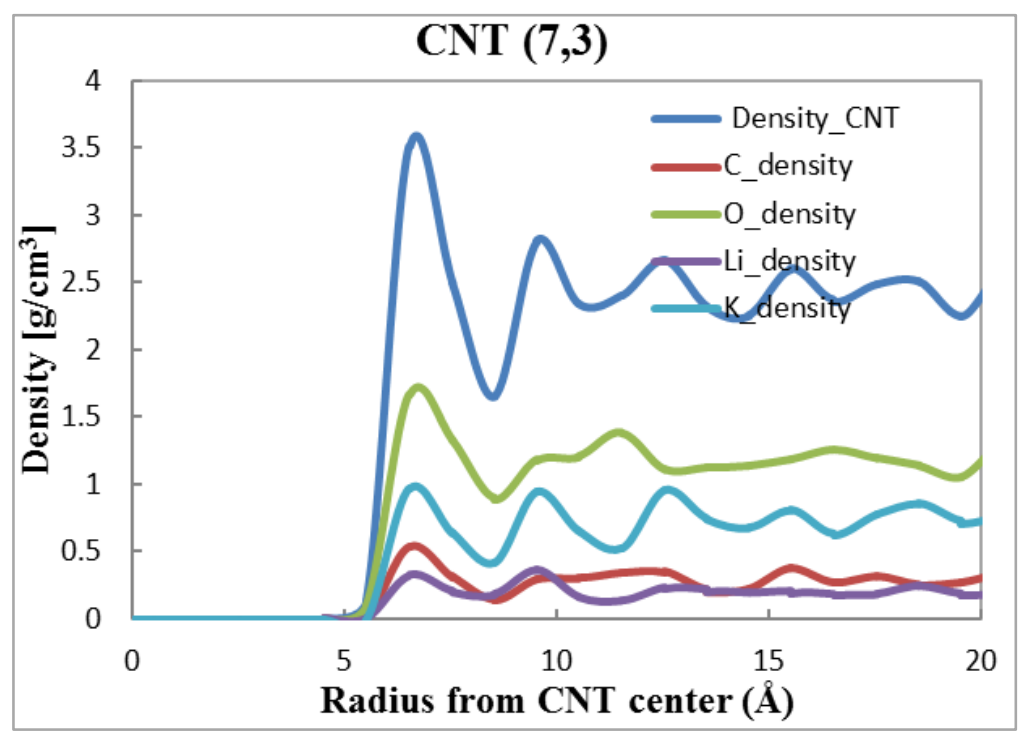

Figure 4.19 Spatial density distribution around a $(7,3)$ chiral SWCNT that is surrounded by molten salts molecules.

A plot of interfacial thermal resistance of $(n, 3)$ chiral SWCNT is shown in Figure 4.21. The plot shows that the interfacial thermal resistance increases as the diameter/size of the $(\mathrm{n}, 3)$ chiral carbon nanotube increases. However, considering that there are both metallic and semiconducting carbon nanotubes for the (n, 2) chiral category, it is 
observed that the metallic carbon nanotubes have significantly higher values of the interfacial thermal resistance compared to that of the semiconducting carbon nanotubes.

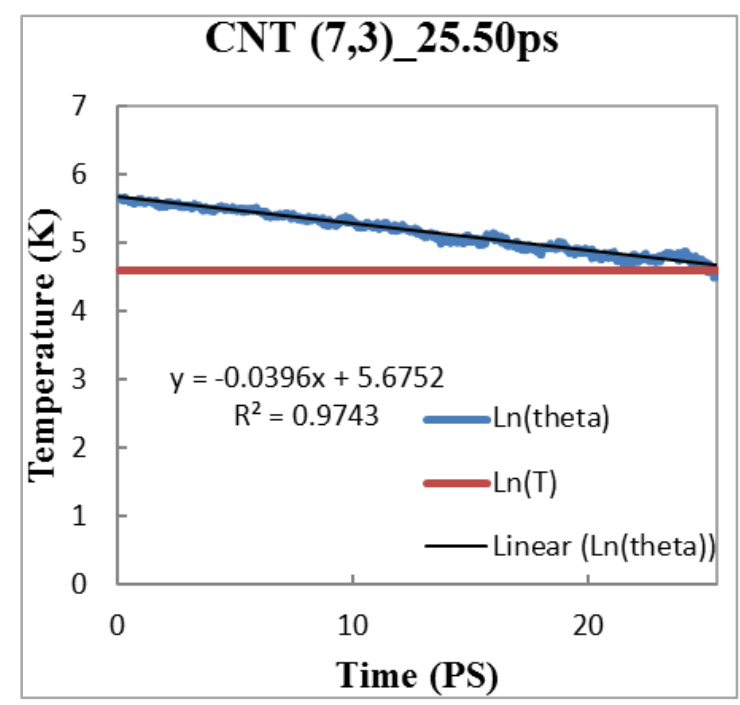

Figure 4.20 Temperature decay plot of a $(7,3)$ chiral SWCNT that is surrounded by molten salts molecules.

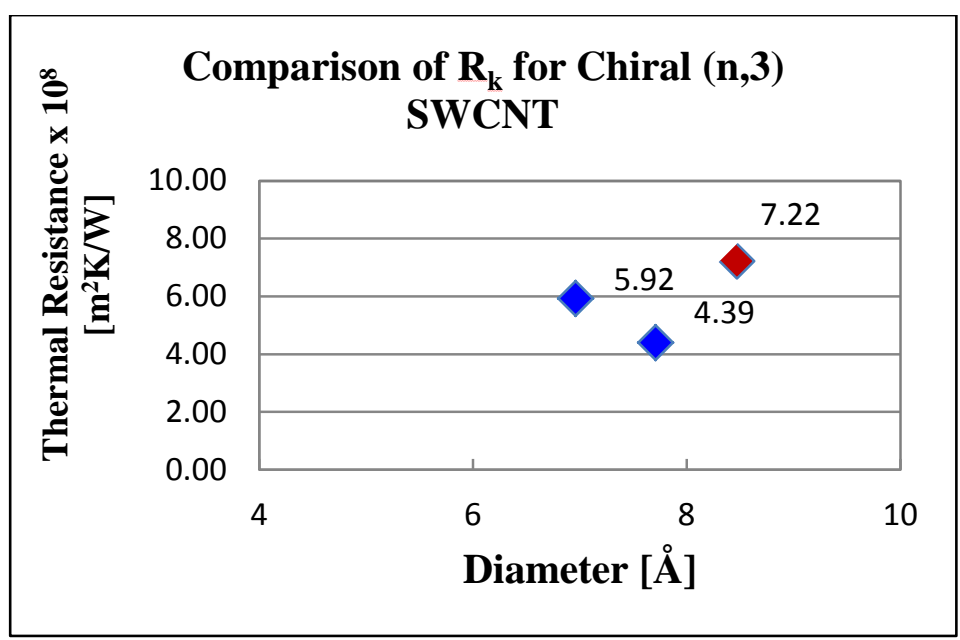

Figure 4.21 Plot of interfacial thermal resistance of (n, 3) Chiral SWCNTs. 
Unlike the simulations involving the armchair and zig-zag carbon nanotubes, the chiral nanotubes are observed to be more susceptible to unstable situations, such as, the disconnected sections of the carbon nanotube occurring during the simulations. This issue will be discussed later in the thesis.

\subsection{4. (n, 4) Chiral SWCNT}

From Figure 1.9 it is observed that the $(n, 4)$ chiral carbon nanotubes are located on the fourth horizontal dotted line, which is right below the (n, 3) dotted line. The calculated values of the interfacial thermal resistance are listed in Table 4.6.

Table 4.6. Interfacial thermal resistance of Chiral (n, 4) SWCNT

\begin{tabular}{|c|c|c|c|c|c|}
\hline SWCNT & Diameter & Length & Property & $\begin{array}{c}\mathrm{R}_{\mathrm{k}} \\
{\left[\mathrm{m}^{2} \cdot \mathrm{K} / \mathrm{W}\right]}\end{array}$ & $\begin{array}{c}\mathrm{d}_{\mathrm{c}} \\
{[\mathrm{nm}]}\end{array}$ \\
\hline$(6,4)$ & $6.83 \AA$ & $\begin{array}{c}55.71 \AA \\
(3 \mathrm{unit})\end{array}$ & Semi-conductor & $5.11 \mathrm{e}-8$ & 76.65 \\
\hline$(7,4)$ & $7.55 \AA$ & $\begin{array}{c}41.08 \AA \\
(1 \mathrm{unit})\end{array}$ & Metallic & $8.48 \mathrm{e}-8$ & 127.2 \\
\hline$(8,4)$ & $8.29 \AA$ & $\begin{array}{c}45.08 \AA \\
(2 \mathrm{unit})\end{array}$ & Semi-conductor & $5.29 \mathrm{e}-8$ & 79.35 \\
\hline
\end{tabular}

Figure 4.22 shows the simulation domain obtained upon the completion of the MD simulations. The figure shows that the $(7,4)$ chiral carbon nanotube is located in the center of the simulation box and compressed phase forms at the interface between the 
carbon nanotube and the solvent molecules. Similar trends were observed for the simulation results from other $(\mathrm{n}, 4)$ chiral carbon nanotubes as well.

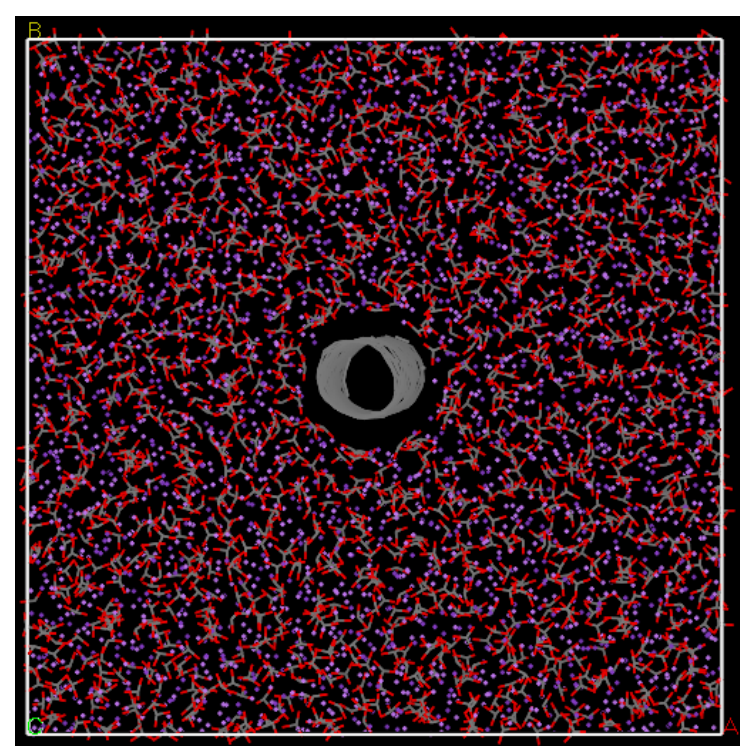

Figure 4.22 Simulation domain showing a $(7,4)$ chiral SWCNT placed in the center and the solvent molecules of carbonate salt eutectic located around the crystal lattice of a nanotube.

Figure 4.23 shows the spatial density distribution of the $(7,4)$ chiral carbon nanotube, where the abscissa represents the radial location starting from the center of the carbon nanotube. Considering that the radius of the $(7,4)$ chiral carbon nanotube is $3.775 \AA$, no molecules exist in a spherical volume with a diameter of $\sim 7.5 \AA$. Figure 4.24 shows the temperature decay plot. Similar trends were observed for simulation results obtained with other (n, 4) chiral carbon nanotubes as well. 


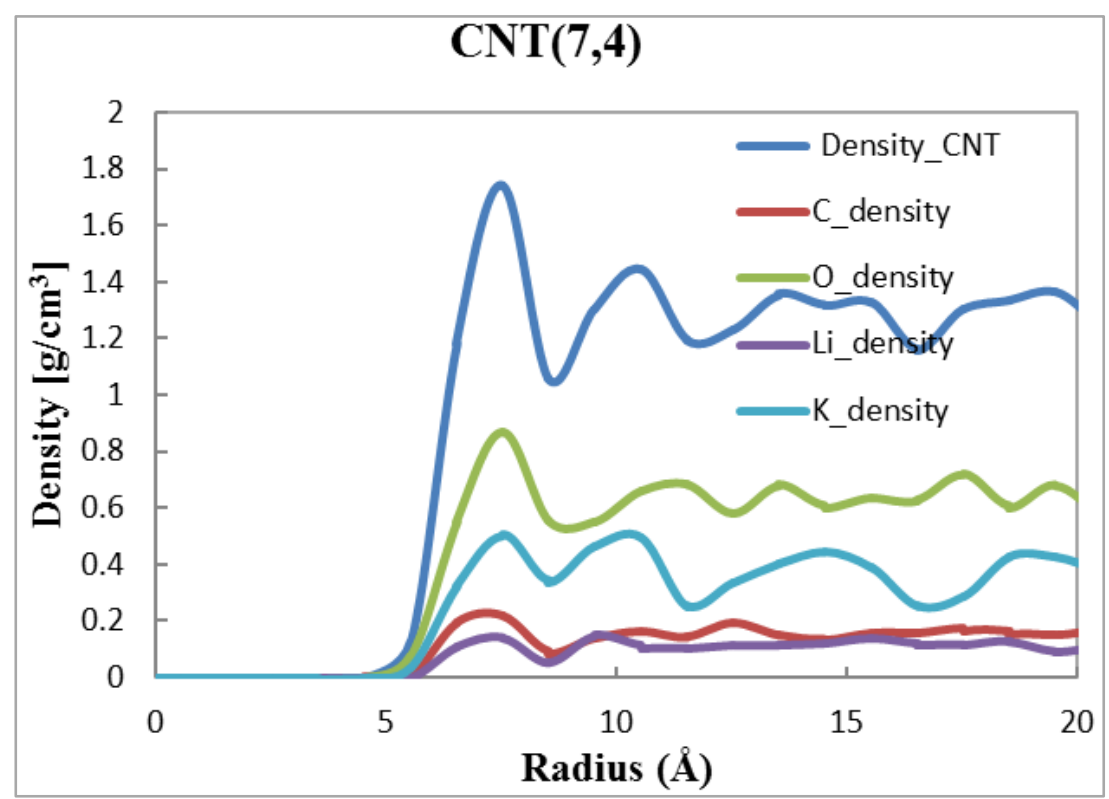

Figure 4.23 Spatial density distribution of a $(7,4)$ chiral SWCNT that is surrounded by molten salts molecules.

The plot in Figure 4.25 shows that the interfacial thermal resistance increases as the diameter/size of the $(n, 4)$ chiral carbon nanotube increases. However, considering that there are both metallic and semiconducting carbon nanotubes for the $(n, 4)$ chiral category, it is observed that the metallic carbon nanotubes have significantly higher values of the interfacial thermal resistance compared to that of the semiconducting carbon nanotubes. However, unlike the simulations involving the armchair and zig-zag carbon nanotubes, the chiral nanotubes are observed to be more susceptible to unstable situations, such as, the disconnected sections of the carbon nanotube occurring during the simulations. This issue will be discussed later in the thesis. 


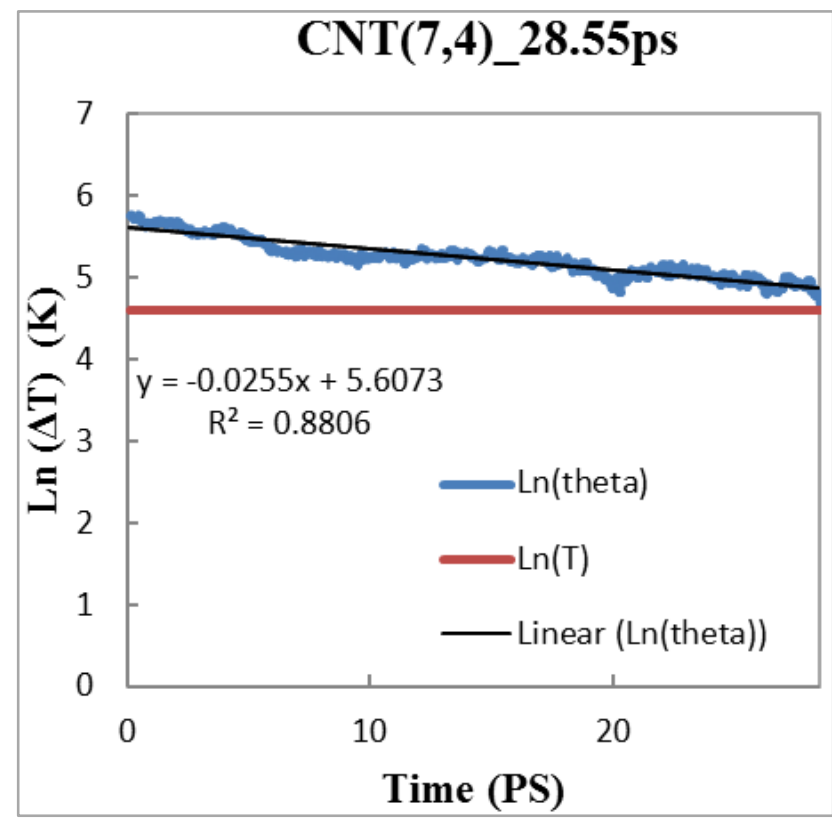

Figure 4.24 Temperature decay plot of a $(7,4)$ chiral SWCNT that is surrounded by molten salts molecules.

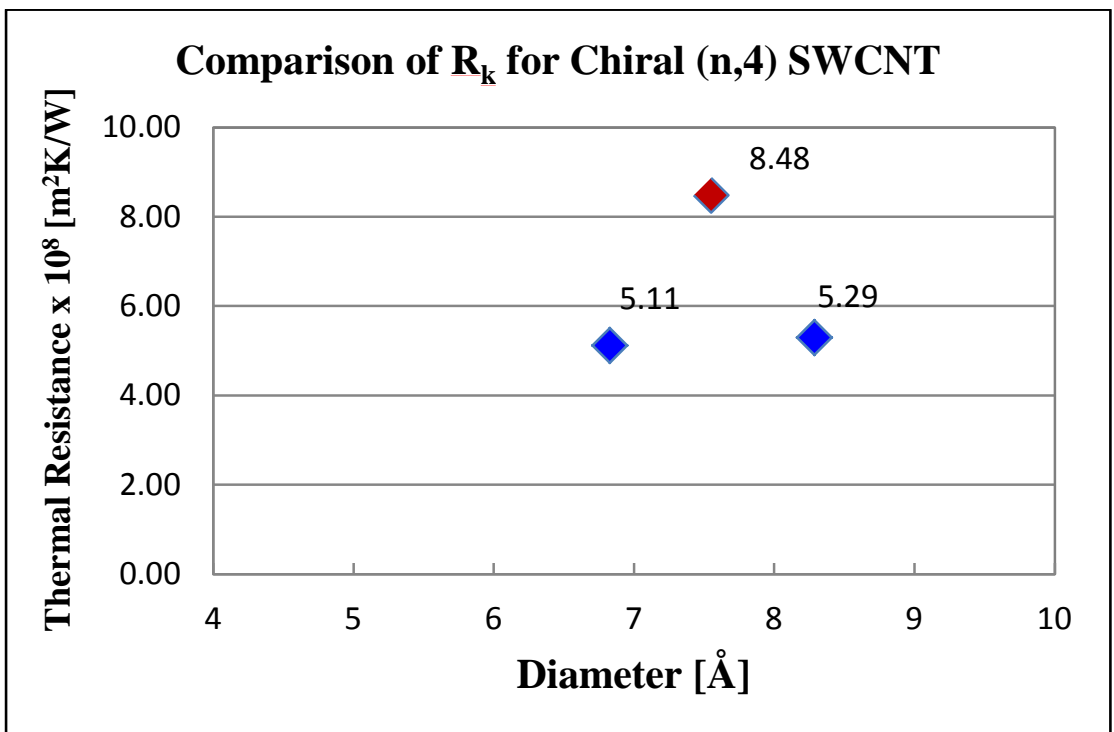

Figure 4.25 Plot of interfacial thermal resistance of (n, 4) Chiral SWCNTs. 


\subsubsection{Defects induced in chiral SWCNT during MD Simulations}

As mentioned in previous sections, stable results are obtained consistently during MD simulations using armchair and zig-zag carbon nanotube. Previous results reported in the literature for MD simulations using the same method are consistent with the results reported in this study, which potentially proves that the methodology employed in these MD simulations is correct. However, for the MD simulations performed using this same technique for cases involving chiral carbon nanotube, several errors and inconsistencies were observed routinely in the simulations, which made it cumbersome for obtaining consistent and correct results.

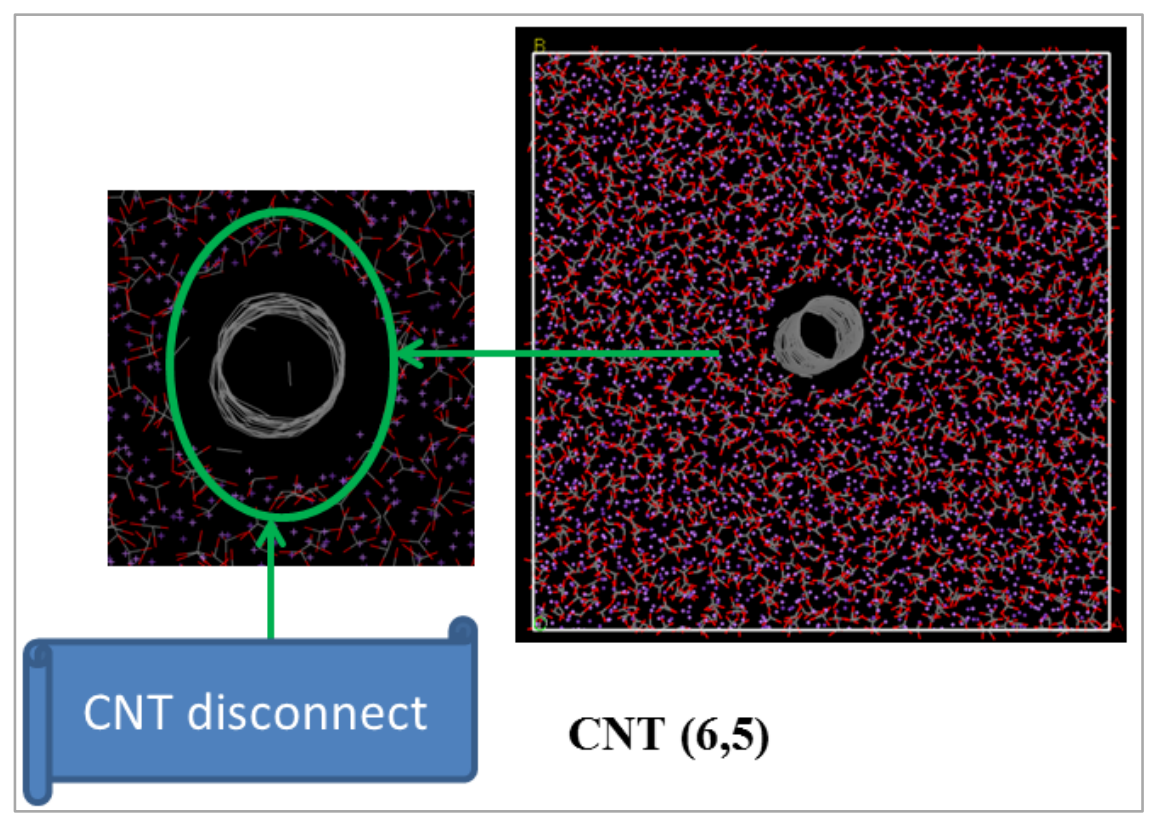

Figure 4.26 Simulation domain showing a $(6,5)$ chiral SWCNT placed in the center and surrounded by solvent molecules of carbonate salt eutectic that are located around the crystal lattice of a nanotube. 
Figure 4.26 shows the case in which the carbon nanotube is disconnected and some of the carbon atoms are dislodged from the carbon nanotube. These types of numerical inaccuracies were never observed for simulations involving armchair and zig-zag carbon nanotube, but happened very frequently for that of the chiral carbon nanotubes. These simulations demonstrated the spatial density oscialliations as shown in Figure 4.27.

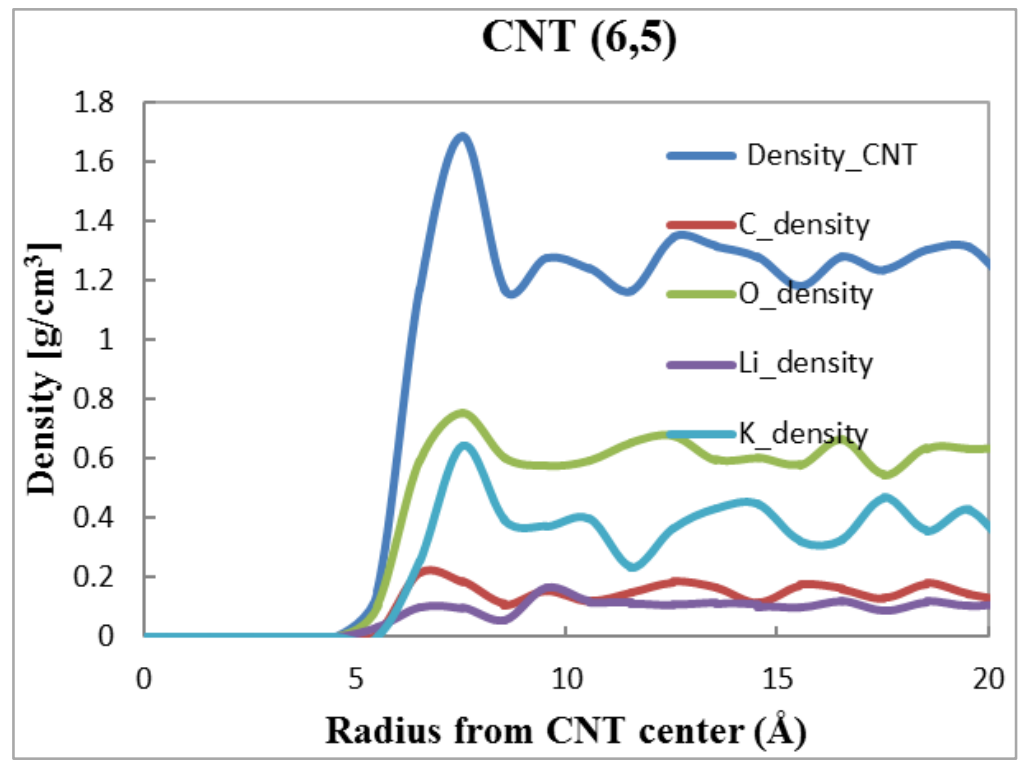

Figure 4.27 Spatial density distribution for a $(6,5)$ chiral SWCNT that is surrounded by molten salts molecules.

However, such a result is potentially erroneous considering the disruption in the structure of the carbon nanotubes. On many occasions, as shown as Figure 4.28, the solvent molecules were lodged inside the carbon nanotube, which in turn predicted results for density plots that were inconsistent, as shown in Figure 4.29. 


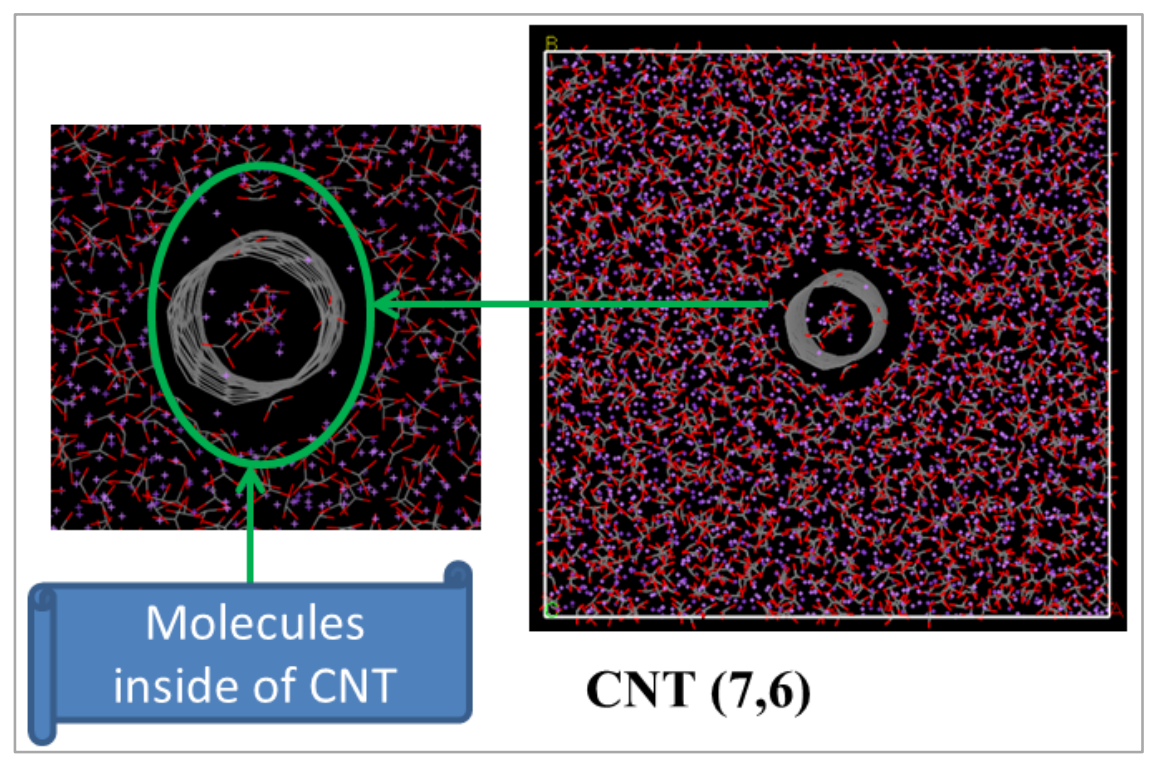

Figure 4.28 Simulation domain showing a $(7,6)$ chiral SWCNT placed in the center and surrounded by solvent molecules of carbonate salt eutectic that are located around a crystal lattice of a nanotube.

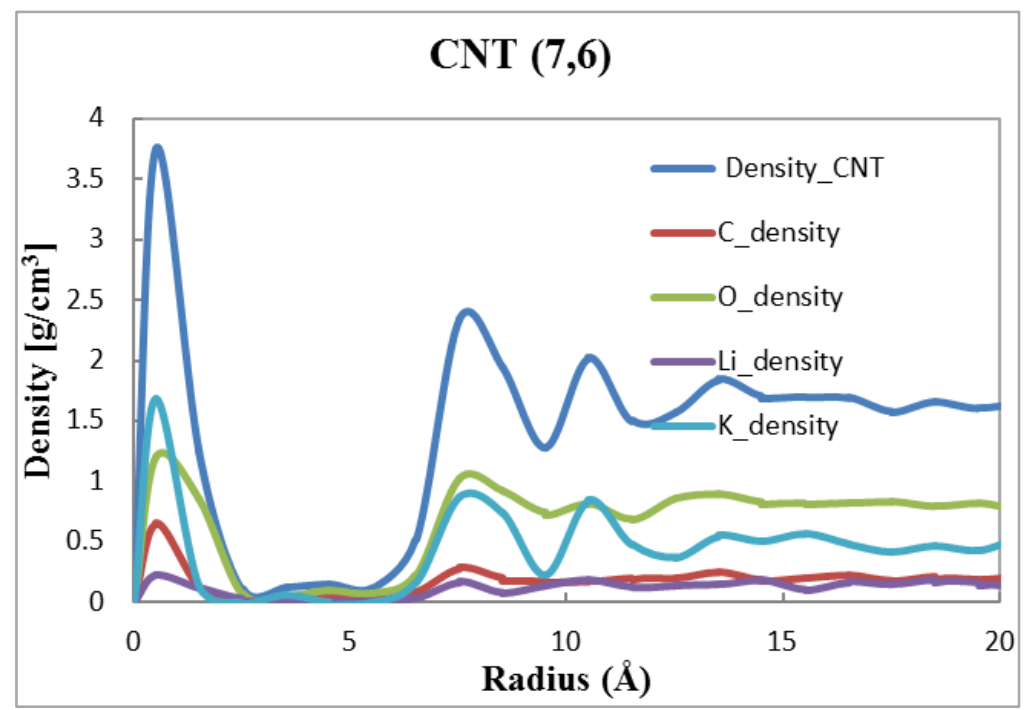

Figure 4.29 Spatial density distribution for a $(7,6)$ chiral SWCNT that is surrounded by molten salts molecules. 
These inaccuracies crept into the simulations involving MD simulations of chiral carbon nanotubes. This is potentially causes by the spatial configuration of the chiral nanotubes that makes the simulation procedure susceptible to numerical instabilities. Considering that almost no experiments were performed exclusively using chiral carbon nanotube, further studies are needed for these types of scenarios to resolve the observed conundrums. Based on these results it may be concluded that chiral carbon nanotubes are probably not a suitable option for heat transfer applications. 


\section{SECTION 5. SUMMARY AND CONCLUSION}

The transient heat loss from a single walled carbon nanotube (SWCNT) into molten salt eutectic (carbonate) has been performed in this study by using Molecular Dynamics (MD) simulations. Three different chirality were considered: armchair, zig-zag, and chiral. The motivation of this study was to examine the impact of the chirality on the values of the interfacial thermal resistance " $R_{k}$ " for a given working fluid (or solvent molecules). The results show an exponential decay in the temporal profile of the temperature for an ensemble of carbon nanotube atoms. The values for the thermal interfacial resistance " $R_{k}$ " between single wall carbon nanotube and eutectic molecules were estimated by using the time constant for the exponential decay.

Based on the simulation results, it has been found that for same type of the chirality, the metallic chirality enhances the value of interfacial thermal resistance by $\sim 50 \%$ compared to that of the semi-conductor chirality. When comparing the three different types of the chirality, for similar values of the carbon nanotube diameter, the metallic zig-zag single wall carbon nanotubes have the higher value of the interfacial thermal resistance compared to that of the armchair nanotubes (since all the armchair nanotubes are metallic) and chiral single wall carbon nanotubes that are metallic. This is the 
significant observation, especially considering the previous studies in the literature which have focused only on a single type of chirality, i.e. armchair carbon nanotube. The larger value of interfacial thermal resistance for metallic carbon nanotube implies the critical particle size of the metallic chirality is higher than that of the semi-conducting chirality (for the purpose of maximizing the thermal conductivity of the mixture, i.e. for nanofluids or nanocomposites). Therefore, there is a more susceptibility for mismatch in the desired size of nanoparticles (i.e., for metallic single wall carbon nanotube) for the purpose of maximizing both thermal conductivity that may be required of coolant materials and the specific heat capacity (that may be required for current CSP/TES applications). Therefore, the semi-conducting carbon nanotubes are expected to provide better performance characteristics by providing lower mismatch between the the critical size range of carbon nanotube in CSP/ TES applications $(\sim 10 \mathrm{~nm})$ when compared to the critical size required for maximizing thermal conductivity ( $\sim 100 \mathrm{~nm}$ ). Hence for the purpose of maximizing both specific heat capacity and thermal conductivity values of the mixture (i.e. both for nano-fluids and nano-composites), chiral nanotubes may be more appropriate. 


\section{SECTION 6. FUTURE DIRECTIONS}

\subsection{Functionalized carbon nanotube}

A number of theoretical investigations for the specific heat capacity of nanomaterial showed that the heat capacity was strongly enhanced dependent on the properties and morphology of the compressed phase (fluid molecules adsorbed on the surface of

crystalline solids). ${ }^{2,3,28,33}$ The concomitant decrease in the nanoparticle size, say for the same mass concentration of the nanoparticles, will cause a significant amplification of the total volume of the compressed layer. The heat capacity of the nano-materials will be amplified on progressive reduction in the size of the nanoparticles. Since a delicate balance is required between the need to maximize both the heat capacity and the thermal conductivity for heat transfer applications, the critical particle size that maximizes specific heat capacity has a lower bound $(\sim 10 \mathrm{~nm})$ while the critical particle size that maximizes thermal conductivity occurs for larger size nanoparticles $(\sim 100 \mathrm{~nm})$. In order to optimize the critical diameter of nanoparticles for simultaneously maximizing the both heat capacity and the thermal conductivity, surface functionalization (i.e., chemical functionalization) of the nanotubes may have some utility. Hence chemical functionalization of the nanoparticles is recommended a future topic for investigation in both experimental and numerical studies. 


\section{REFERENCES}

1. P. Hodgson. Modern Age. 50, 238, (2008).

2. D. Shin. Molten Salt Nanomaterials for Thermal Energy Storage and Concentrated Solar Power Applications Ph.D. Dissertation. Texas A\&M University (2011).

3. B. Jo. Numerical and Experimental Investigation of Organic Nanomaterials for Thermal Energy Storage and for Concentrating Solar Power Applications Ph.D. Dissertation. Texas A\&M University (2012).

4. Neil. Molten Salt for Heat Storage, Renewable Energy United Kingdom website, (2014). <http://www.reuk.co.uk/Molten-Salt-for-Heat-Storage.htm>

5. C. Koroneos, T. Spachos and N. Moussiopoulos, Renewable Energy. 28, 295-310, (2003).

6. D. Kearney, U. Herrmann, B. Kelly, R. Mahoney, R. Cable, D. Blake, Journal of Solar Energy Engineering. 125, 170-176, (2003).

7. N. Araki, M. Matsuura, A. Makino, T. Hirata and Y. Kato, Int.J.Thermophys. 9, 1071-1080, (1988). 
8. S. Iijima. Nature. 354, 56, (1991).

9. P. Ma, N. A. Siddiqui, G. Marom and J. Kim, Composites Part A: Applied Science and Manufacturing. 41, 1345-1367, (2010).

10. D. S. Bethune, C. -. Kiang, M. S. de Vries, G. Gorman, R. Savoy, J. Vazquez, Nature. 363, 605-607, (1993).

11. Y. Hu and D. Banerjee, Journal of Nanofluids. 2, 29-37, (2013).

12. M. S. Dresselhaus, G. Dresselhaus and R. Saito, Carbon. 33, 883-891, (1995).

13. T. Chou, E. T. Thostenson and L. Gao, Advances in the Science and Technology of Carbon Nanotube Composites, Proceedings of the 17th International Conference on Composite Materials, (2009) July 27-29; Edinburgh, United Kingdom.

14. W. Minkowycz, E. M. Sparrow and J. P. Abraham, Nanoparticle Heat Transfer and Fluid Flow, 4, CRC Press, Taylor \& Francis Group, United States, (2012).

15. S. Kakaç and A. Pramuanjaroenkij, Int.J.Heat Mass Transfer. 52, 3187-3196, (2009).

16. P. Zhou, B. Wu, Y. Hu, CFD Study for Air Distribution in Hydrogen Reformer Furnace, Proceedings of the ASME 2009 Heat Transfer Summer Conference collocated with the InterPACK09 and 3rd Energy Sustainability Conferences, (2009) July 19-23; San Francisco, CA. 
17. B. Wu, D. Zheng, N. Chen, C. Zhou and D. Lu, Iron \& Steel Technology. 8, 41-50, (2011).

18. Y. Hu. Computational Fluid Dynamics Simulation and Virtual Reality visualization of a Blast Furnace Hearth Master Thesis. Purdue University Calumet (2011).

19. D. Zheng, S. Zhang, B. Wu, J. Fleitz, R. Trajkovski and C. Q. Zhou, CFD Investigation of a Hydrogen Reformer Furnace for Improved Flow Distribution, Proceedings of the ASME 2010 International Mechanical Engineering Congress and Exposition, (2010) November 12-18; Vancouver, British Columbia, Canada.

20. D. Zheng, B. Wu, J. Fleitz, R. Trajkovski and C. Q. Zhou, CFD Simulation of a Hydrogen Reformer Furnace, Proceedings of the 2010 14th International Heat Transfer Conference, (2010) August 7-13; Washington D.C.

21. D. Fu, B. Wu, G. Chen, Virtual Reality Visualization of CFD Simulation for Iron/Steelmaking Processes, Proceedings of the 14th International Heat Transfer Conference, (2010) August 7-13; Washington D.C.

22. D. Zheng. CFD Simulation and VR Visualization for Industrial Applications Master Thesis. Purdue University Calumet (2011).

23. B. Wu, G. Chen, J. Moreland, D. Huang, D. Zheng and C. Q. Zhou, Industrial Application of CFD Simulation and VR Visualization, Proceedings of the ASME 2010 World Conference on Innovative Virtual Reality, (2010) May 12-14; Ames, IA. 
24. D. Fu, D. Zheng, C. Q. Zhou, J. D’Alessio, K. J. Ferron and Y. Zhao, Parametric Studies on PCI Performances, Proceedings of the ASME/JSME 2011 8th Thermal Engineering Joint Conference, (2011) March 13-17; Honolulu, HI.

25. S. Shenogin. J.Appl.Phys. 95, 8136-8144, (2004).

26. S. T. Huxtable. Nature Materials. 2, 731, (2003).

27. S. H. Oh, Y. Kauffmann, C. Scheu, W. D. Kaplan and M. Ruhle, Science. 310, 661-663, (2005).

28. S. Jung. Numerical and Experimental Investigation of Inorganic Nanomaterials for Thermal Energy Storage (TES) and Concentrated Solar Power (CSP) Applications Ph.D. Dissertation. Texas A\&M University (2012).

29. B. Jo and D. Banerjee, Interfacial Thermal Resistance between a Carbon Nanoparticle and Molten Salt Eutectic: Effect of Material Properties, Particle Shapes and Sizes, Proceedings of the ASME/JSME 2011 8th Thermal Engineering Joint Conference, (2011) March 13-17; Honolulu, HI.

30. H. S. Ahn, N. Sinha, M. Zhang, D. Banerjee, S. Fang and R. H. Baughman, Journal of Heat Transfer. 128, 1335, (2006).

31. S. R. Sriraman. Pool Boiling on Nano-finned Surfaces Master Thesis. Texas A\&M University (2007). 
32. S. Ujereh, I. Mudawar, P. B. Amama, T. S. Fisher and W. Ou, American Society of Mechanical Engineers, Heat Transfer Division. 691-696, (2005).

33. N. Singh, V. Unnikrishnan, D. Banerjee and J. Reddy, International Journal for Computational Methods in Engineering Science and Mechanics. 12, 254, (2011).

34. R. S. Ruoff. Carbon. 33, 925, (1995).

35. S. Berber, Y. Kwon and D. Tomanek, Phys.Rev.Lett. 84, 4613-4616, (2000).

36. M. J. Biercuk. Appl.Phys.Lett. 80, 2767-2769, (2002).

37. I. Nelson. Characterization of thermo-physical properties and forced convective heat transfer of poly-alpha-olefin (PAO) nanofluids Master Thesis. Texas A\&M University (2007).

38. H. S. Ahn, V. Sathyamurthi and D. Banerjee, Components and Packaging Technologies, IEEE Transactions on. 32, 156-165, (2009).

39. V. Sathyamurthi, H. Ahn, D. Banerjee and S. Lau, Journal of Heat Transfer. 131, 071501, (2009).

40. N. Singh, V. Sathyamurthy, W. Peterson, J. Arendt and D. Banerjee, Int J Heat Fluid Flow. 31, 201-207, (2010). 
41. S. Jeon, B. Jo and D. Banerjee, Enhancement of Saturation Boiling of PF-5060 on Microporous Surface, Proceedings of the ASME/JSME 2011 8th Thermal Engineering Joint Conference, (2011) March 13-17; Honolulu, HI.

42. V. Unnikrishnan, D. Banerjee and J. Reddy, International Journal of Thermal Sciences. 47, 1602-1609, (2008).

43. N. Singh. Computational Analysis of Thermo-fluidic Characteristics of a Carbon nano-fin Ph.D. Dissertation. Texas A\&M University (2011).

44. I. C. Nelson, D. Banerjee and R. Ponnappan, J.Thermophys.Heat Transfer. 23, 752-761, (2009).

45. D. Shin and D. Banerjee, The International Journal of Structural Changes in Solids. 2, 25-31, (2011).

46. D. Shin and D. Banerjee, Journal of Heat Transfer. 133, (2011).

47. D. Shin and D. Banerjee, Int.J.Heat Mass Transfer. 54, 1064-1070, (2011).

48. B. Jo and D. Banerjee, Study of High Temperature Nanofluids using Carbon Nanotubes (CNT) for Solar Thermal Storage Applications, Proceedings of the ASME 2010 4th International Conference on Energy Sustainability, (2010) May 17-22; Phoenix, AZ. 
49. D. Shin and D. Banerjee, Investigation of Nanofluids for Solar Thermal Storage Applications, Proceedings of the ASME 3rd International Conference on Energy Sustainability, (2009) July 19-23; San Francisco, CA.

50. D. Shin and D. Banerjee, Enhanced Thermal P Roperties of Pcm Based Nanofluid for Solar Thermal Energy Storage, Proceedings of the ASME 2010 4th International Conference on Energy Sustainability, (2010) May 17-22; Phoenix, AZ.

51. D. Shin and D. Banerjee, SAE Technical Paper. 01-1734, (2010).

52. D. Shin and D. Banerjee, Experimental Investigation of Molten Salt Nanofluid for Solar Thermal Energy Application, Proceedings of the ASME/JSME 2011 8th Thermal Engineering Joint Conference, (2011) March 13-17; Honolulu, HI.

53. H. Kwak, D. Shin and D. Banerjee, Enhanced Sensible Heat Capacity of Molten Salt and Conventional Heat Transfer Fluid Based Nanofluid for Solar Thermal Energy Storage Application, Proceedings of the ASME 2010 4th International Conference on Energy Sustainability, (2010) May 17-22; Phoenix, AZ.

54. S. Jung and D. Banerjee, Enhancement of Heat Capacity of Nitrate Salts using Mica Nanoparticles, Developments in Strategic Materials and Computational Design II - 35th International Conference on Advanced Ceramics and Composites, (2011) January 23-28; Daytona Beach, FL. 
55. B. Jo and D. Banerjee, Enhanced Specific Heat Capacity of Nanocomposites using Organic Nanoparticles, Proceedings of ASME 2011 International Mechanical Engineering Congress and Exposition, (2011) November 11-18; Denver, CO.

56. B. Jo and D. Banerjee, Enhanced Viscosity of Aqueous Silica Nanofluids, Proceeding of the Developments in Strategic Materials and Computational Design II 35th International Conference on Advanced Ceramics and Composites, (2011) January 23-28; Daytona Beach, FL.

57. B. Jo, S. Jung, D. Shin and D. Banerjee, Anomalous Rheological Behavior of Complex Fluids (Nanofluids), Proceedings of the ASME 2011 International Mechanical Engineering Congress and Exposition, (2011) November 11-18; Denver, CO.

58. S. Jung, B. Jo, D. Shin and D. Banerjee, SAE Technical Paper. 01-1731, (2010).

59. S. Jung and D. Banerjee, A Simple Analytical Model for Specific Heat of Nanofluid with Tube Shaped and Disc Shaped Nanoparticles, Proceedings of the ASME/JSME 2011 8th Thermal Engineering Joint Conference, (2011) March 13-17; Honolulu, HI.

60. J. Yu, S. Kang, S. Jeon and D. Banerjee, Frontiers in Heat and Mass Transfer. 3, 013004, (2012).

61. M. Sunder and D. Banerjee, Int J Heat Fluid Flow. 30, 140-149, (2009).

62. N. Sinha, H. S. Ahn, R. Williams and D. Banerjee, IEEE Transactions on Components and Packaging Technologies. 32, 252-260, (2009). 
63. V. Sathyamurthi and D. Banerjee, Int.J.Heat Mass Transfer. 52, 5608-5623, (2009).

64. L. Li. The Journal of Physical Chemistry.B. 110, 10509-13, (2006).

65. L. A. Girifalco, M. Hodak and R. S. Lee, Physical Review B (Condensed Matter). 62, 13104-10, (2000).

66. T. Werder, J. H. Walther, R. L. Jaffe, T. Haliciogiu, F. Noca and P. Koumoutsakos, Nano Letters. 1, 697-702, (2001).

67. T. A. Adams. Physical Properties of Carbon Nanotubes, Michigan State University, (2000). $<h t t p: / / w w w . p a . m s u . e d u / c m p / c s c / n t p r o p e r t i e s / m a i n . h t m l \# i n t r o>$ 